\title{
ASYMPTOTICS OF GEOMETRICAL NAVIGATION ON A RANDOM SET OF POINTS IN THE PLANE
}

\author{
NICOLAS BONICHON ${ }^{* * *}$ AND \\ JEAN-FRANÇOIS MARCKERT, ${ }^{* * *}$ Université de Bordeaux
}

\begin{abstract}
A navigation on a set of points $S$ is a rule for choosing which point to move to from the present point in order to progress toward a specified target. We study some navigations in the plane where $S$ is a nonuniform Poisson point process (in a finite domain) with intensity going to $+\infty$. We show the convergence of the traveller's path lengths, and give the number of stages and the geometry of the traveller's trajectories, uniformly for all starting points and targets, for several navigations of geometric nature. Other costs are also considered. This leads to asymptotic results on the stretch factors of random Yao graphs and random $\theta$-graphs.
\end{abstract}

Keywords: Navigation; Poisson point process; greedy routing; spatial network; proximity graph

2010 Mathematics Subject Classification: Primary 60D05; 68W40; 60B10; 60G55

\section{Introduction}

\subsection{Navigations}

Often a traveller, which can be a person, a migratory animal, a letter, a radio message, a message in a wireless ad-hoc network, etc., wanting to reach a point $t$ starting from a point $s$ has to stop along the route in order to, depending on the type of traveller, sleep, eat, be sorted, be amplified, or routed. Generally, the traveller cannot stop anywhere: only some special places offer what is needed (a hostel, a river, a post office, a radio relay station, a router, etc.). Often the traveller cannot compute the optimal route from its initial position: the traveller must choose the next point to move to using only some local information. In this paper we deal with this problem, that is, we address the following questions. Given a random set $S$ of possible stops, what happens if a traveller stops 'at the first point in $S$ ' which is in the direction of $t$ up to an angle $\theta$ ? How many stops are necessary? What is the total distance covered? In this paper we answer these questions in the asymptotic case, when the number of points in $S$ goes to $+\infty$.

Formally, consider a traveller on $\mathbb{R}^{2}$ beginning its travel at the starting position $s$ and wanting to reach the target $t$ using as a set of possible stopping places $S$, a finite subset of $\mathbb{R}^{2}$. Navigation (also called the memoryless routing algorithm in the literature) with a set of stopping places $S$ is defined as a function $\boldsymbol{X}: \mathbb{R}^{2} \times \mathbb{R}^{2} \rightarrow \mathbb{R}^{2}$ such that, for any $(s, t), \boldsymbol{X}(s, t)$ belongs to $S \cup\{t\}$ and, moreover, satisfies $\boldsymbol{X}(s, s)=s$ for any $s \in \mathbb{R}^{2}$. The position $\boldsymbol{X}(s, t)$ corresponds to the

Received 21 October 2010; revision received 20 May 2011.

* Postal address: CNRS, LaBRI, Université de Bordeaux, 351 cours de la Libération, 33405 Talence cedex, France.

** Email address: bonichon@labri.fr

Partially supported by the ANR project ALADDIN.

*** Email address: marckert@labri.fr

Partially supported by ANR-08-BLAN-0190-04 A3. 
traveller's first stop between $s$ and $t$. Hence,

$$
\boldsymbol{X}(s, t, j):=\boldsymbol{X}(\boldsymbol{X}(s, t, j-1), t), \quad j \geq 1,
$$

are the successive stops of the traveller, where, by convention, $\boldsymbol{X}(s, t, j)=s$ for $j=0$. We write $s_{j}$ instead of $\boldsymbol{X}(s, t, j)$ when no confusion is possible on $s, t$, and $\boldsymbol{X}$. The quantity

$$
\Delta^{X}(s, t, j):=X(s, t, j)-X(s, t, j-1)
$$

is called the $j$ th stage. For a general navigation algorithm $\boldsymbol{X}$, if $\# S<+\infty$, either $\boldsymbol{X}(s, t, j)=t$ for large enough $j$ or $\boldsymbol{X}(s, t, j) \neq t$ for all $j$. In the first case, the global navigation from $s$ to $t$ succeeds, whereas in the second case, it fails. In the case of success, the (global) path from $s$ to $t$ is

$$
\operatorname{Path}^{X}(s, t):=\left(s_{0}, \ldots, s_{\mathrm{Nb}^{X}(s, t)}\right),
$$

where $\mathrm{Nb}^{X}(s, t):=\min \left\{j, s_{j}=t\right\}$ is the number of stages needed to go from $s$ to $t$.

We are interested in navigations in $\mathbb{R}^{2}$ where the point to move to is chosen according to some geometric rules: we consider two classes of so-called compass navigations; in these navigations the next stopping place to move to is selected as the 'nearest' point of $s$ in the set $S \cup\{t\}$, in the 'direction' of $t$ (see Section 1.2).

Throughout the paper, $\mathscr{D}$ refers to a bounded and simply connected open domain in $\mathbb{R}^{2}$. The sets of considered stopping places $S$ are finite random subsets of $\mathscr{D}$ taken according to two models: $S$ will be either the set $\left\{p_{1}, \ldots, p_{n}\right\}$, where the $p_{i}$ points are picked independently according to a distribution with regular density $f$ (with respect to the Lebesgue measure) in $\mathscr{D}$ (see Section 2.3.2), or $S$ will be a Poisson point process with intensity $n f$ for some $n>0$ (see Section 1.3).

The main goal of this paper is to study the global asymptotic behaviour of the paths of the traveller. Global means that all possible trajectories corresponding to all starting points $s$ and targets $t$ of $\mathscr{D}$ are considered together. Several quantities are then studied that describe the 'deviation' of the paths of the traveller (or functionals of the path, such as the length) to a deterministic limit (see Section 1.4). We also give the asymptotic of the number of points of $S$, which goes to $+\infty$ (equivalently $n \rightarrow+\infty$ in one of the models).

Convention. Throughout the paper, the two-dimensional real plane $\mathbb{R}^{2}$ is identified with the set of complex numbers $\mathbb{C}$ and, according to what appears simpler, either the complex or real notation is used without warning. The real part, the imaginary part, and the modulus of $z$ are respectively written as $\operatorname{Re}(z), \operatorname{Im}(z)$, and $|z|$; the $\operatorname{argument} \arg (z)$ of any real number $z \neq 0$ is the unique real number $\theta$ in $[0,2 \pi)$ such that $z=\rho \mathrm{e}^{\mathrm{i} \theta}$ for some $\rho>0$ (we set $\arg (0)=0$ ). The characteristic function of the set $A$ is denoted by $\mathbf{1}_{A}$. The notation $\llbracket x, y \rrbracket$ refers to the set of integers included in $[x, y]$. The open ball with centre $x$ and radius $r$ in $\mathbb{C}$ is denoted by $B(x, r)=\{y \in \mathbb{C},|x-y|<r\}$ and the closed ball is denoted by $\bar{B}(x, r)$. For $x \in \mathbb{C}$ and $A \subset \mathbb{C}, d(x, A)=\inf \{|x-y|, y \in A\}$.

\subsection{Two types of navigation}

The two types of navigation introduced below, cross navigation and straight navigation, may appear very similar, but their asymptotic behaviours as well as their analysis are quite different. 
For any $\beta \in(0,2 \pi)$, as illustrated in Figure 1, let

$$
\begin{aligned}
\operatorname{Sect}(\beta) & :=\left\{\rho \mathrm{e}^{\mathrm{i} v}, \rho>0,|\nu| \leq \frac{1}{2} \beta\right\}, \\
\operatorname{Cam}(\beta)(h) & :=\operatorname{Sect}(\beta) \cap \bar{B}(0, h), \\
\operatorname{Tri}(\beta)(h) & :=\left\{x+\mathrm{i} y, x \in(0, h], y \in \mathbb{R},|y| \leq x \tan \left(\frac{1}{2} \beta\right)\right\} .
\end{aligned}
$$

The notation Cam and Tri are short for 'Camembert portion' and 'triangle'.

1.2.1. First type of navigation: cross navigation. Cross navigations are parametrised by a parameter $\theta$ satisfying $\theta=2 \pi / p_{\theta}$ for some $p_{\theta} \in\{3,4, \ldots\}$. For any $x \in \mathbb{C}$ and $\kappa$ in $\llbracket 0, p_{\theta}-1 \rrbracket$, the $\kappa$ th angular sector around $x$ is

$$
\operatorname{Sect}[\kappa, x]:=x+\mathrm{e}^{\mathrm{i} \kappa \theta} \operatorname{Sect}(\theta) .
$$

The two half-lines $\mathrm{HL}_{\kappa}(x)$ and $\mathrm{HL}_{\kappa+1}(x)$ defined by

$$
\mathrm{HL}_{j}(x):=x+\left\{\rho \mathrm{e}^{\mathrm{i} \theta(j-1 / 2)}, \rho>0\right\}, \quad j \in \llbracket 0, p_{\theta} \rrbracket,
$$

are called the first border and last border of Sect $[\kappa, x]$. As illustrated in Figure 2, for $h>0$, the $\kappa$ th triangle and $\kappa$ th Camembert section around $z$ with height $h$ are respectively

$$
\operatorname{Tri}[\kappa, x](h):=x+\mathrm{e}^{\mathrm{i} \kappa \theta} \operatorname{Tri}(\theta)(h), \quad \operatorname{Cam}[\kappa, x](h):=x+\mathrm{e}^{\mathrm{i} \kappa \theta} \operatorname{Cam}(\theta)(h),
$$

where, for any $z_{1}, z_{2} \in \mathbb{C}$ and any $A \subset \mathbb{C}, z_{1}+z_{2} A$ is the set $\left\{z_{1}+z_{2} y, y \in A\right\}$.

As can be seen from Figure 2, the half-lines $\left(\mathrm{HL}_{\kappa}(t), \kappa \in \llbracket 1, p_{\theta} \rrbracket\right)$ form a cross around $t$ that we denote by $\operatorname{Cross}(t):=\bigcup_{\kappa=1}^{p_{\theta}} \mathrm{HL}_{\kappa}(t)$. This justifies the terminology 'cross navigation'.
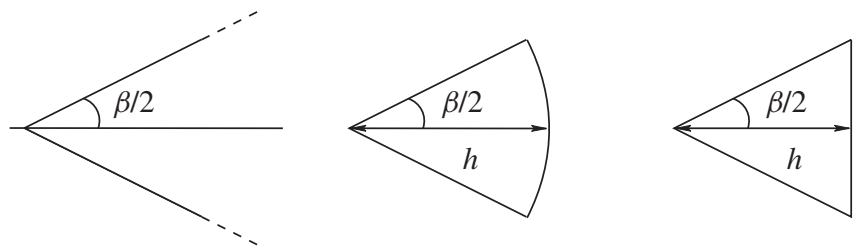

Figure 1: Representations of $\operatorname{Sect}(\beta), \operatorname{Tri}(\beta)(h)$, and $\operatorname{Cam}(\beta)(h)$. Note that each peak point of the diagrams is excluded from the corresponding set.

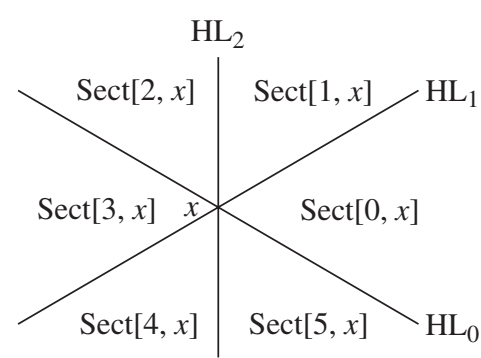

(a)

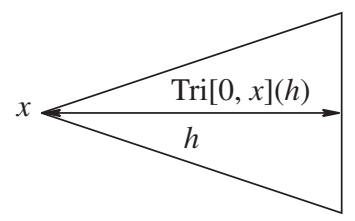

(b)

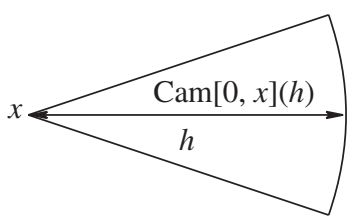

(c)

FigURE 2: Representations of (a) the six angular sectors around a point $x$ with $p_{\theta}=6$, (b) $\operatorname{Tri}[0, x](h)$, and (c) $\operatorname{Cam}[0, x](h)$. 
Let $s$ and $t$ be in $\mathscr{D}$, and let $\kappa$ be such that $t \in \operatorname{Sect}[\kappa, s]$. Consider $\boldsymbol{B}_{\kappa}(s)=\left\{s+r \mathrm{e}^{\mathrm{i} \kappa \theta}, r \geq 0\right\}$ to be the bisecting half-line of $\operatorname{Sect}[\kappa, s]$. Consider the lines parallel to $\operatorname{HL}_{\kappa}(s)$ and $\operatorname{HL}_{\kappa+1}(s)$ passing via $t$. These two lines intersect the half-line $\boldsymbol{B}_{\kappa}(s)$ in two points. The point which is the closest to $s$ is denoted by $I(s, t)$; see Figure 3. The compact $\Gamma(s, t)$ is given by

$$
\Gamma(s, t):=[s, I(s, t)] \cup[I(s, t), t] .
$$

We now define, given a set of stopping places $S$ and a finite subset of $\mathbb{R}^{2}, p_{\theta}$-Yao navigation and $p_{\theta}$-CT navigation, abbreviated as $\mathrm{CY}$ and CT navigation.

Definition 1. (CY navigation.) For $(s, t) \in \mathbb{R}^{2} \times \mathbb{R}^{2}$, consider the smallest integer $\kappa$ in $\llbracket 0, p_{\theta}-$ 1] such that $t$ lies in $\operatorname{Sect}[\kappa, s]$. Consider the smallest $r$ such that $\operatorname{Cam}[\kappa, s](r) \cap(S \cup\{t\})$ is not empty. We set $\mathrm{CY}(s, t)=z$, the element of $\operatorname{Cam}[\kappa, s](r) \cap(S \cup\{t\})$ that is the closest of the first border of Sect $[\kappa, s]$ (see Figure 4(a)).

This navigation appears to be the canonical navigation in Yao's graphs [19].

Definition 2. (CT navigation.) $p_{\theta}$-CT navigation is a simple variant of $p_{\theta}$-Yao navigation. It is defined as $p_{\theta}$-Yao navigation, except that $\operatorname{Tri}[\kappa, s](r)$ replaces $\operatorname{Cam}[\kappa, s](r)$.

Remark 1. If $t$ belongs to $\mathrm{HL}_{\kappa}(s)$ then $t$ lies in both $\operatorname{Sect}[\kappa, s]$ and $\operatorname{Sect}\left[\kappa-1 \bmod p_{\theta}, s\right]$; in the other cases, $t$ belongs to a unique sector $\operatorname{Sect}[\kappa, s]$. In the sequel, the domains $\operatorname{Cam}[\kappa, s](r)$

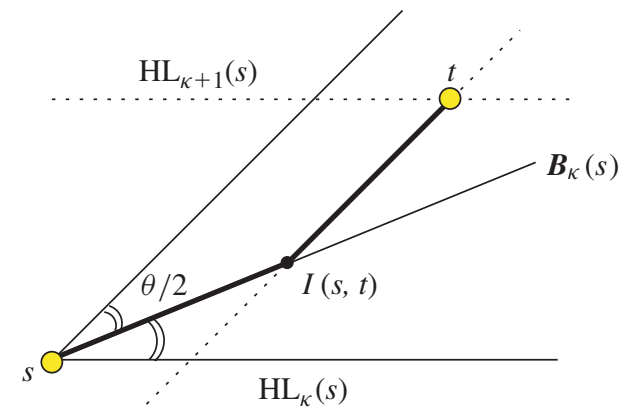

FIGURE 3: Representations of $I(s, t)$ and $\boldsymbol{B}_{\kappa}(s)$. The thick lines denote $\Gamma(s, t)$.

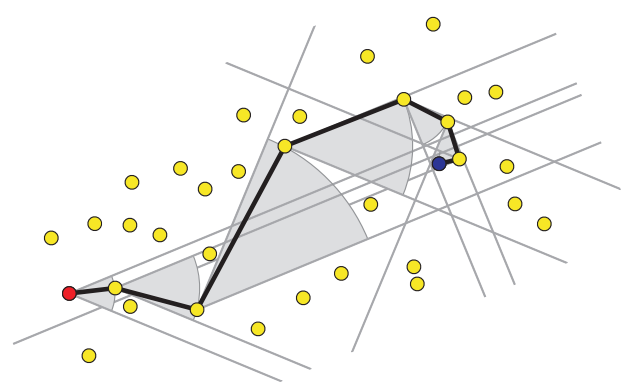

(a)

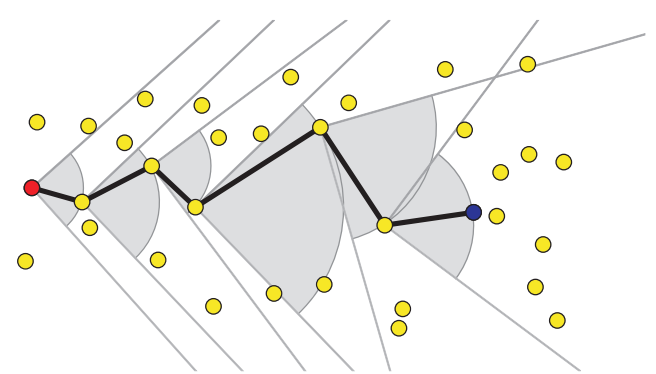

(b)

Figure 4: (a) An example of Yao navigation with $p_{\theta}=8$; the starting point is on the left. (b) An example of Yao straight navigation with $\theta=\pi / 2$; the starting point is on the left. In both examples we give the decision domains for each stage and the sectors that contain the destination. 
and $\operatorname{Tri}[\kappa, s](r)$, which, depending on the algorithm considered, are the minimum domains that the traveller uses to decide which point to move to, are called the decision domains.

1.2.2. Second type of navigation: straight navigation. Straight navigations are closer to real traveller's strategies: the stopping places are chosen not too far from the segment leading to the target. We set two straight navigations, parametrised by a real number $\theta \in(0,2 \pi)$. The difference between the following objects and those previously defined is that the sectors are oriented such that their bisecting lines are the straight line $(s, t)$ :

$$
\begin{aligned}
\overrightarrow{\operatorname{Sect}}(s, t) & :=s+\exp (i \arg (t-s)) \operatorname{Sect}(\theta), \\
\overrightarrow{\operatorname{Cam}}(s, t)(h): & =s+\exp (i \arg (t-s)) \operatorname{Cam}(\theta)(h), \\
\overrightarrow{\operatorname{Tri}}(s, t)(h): & =s+\exp (i \arg (t-s)) \operatorname{Tri}(\theta)(h) .
\end{aligned}
$$

We will use the abbreviations SY and ST to denote $\theta$-straight Yao and $\theta$-straight T navigation, respectively.

Definition 3. (SY navigation.) For $(s, t) \in S^{2}$, consider the smallest $r$ such that the set $\overrightarrow{\mathrm{Cam}}(s, t)(r) \cap(S \cup\{t\})$ is not empty. Set $\operatorname{SY}(s, t)=z$, where $z$ is the closest of the first border of $\overrightarrow{\mathrm{Cam}}(s, t)$ (see Figure 4(b)).

Definition 4. (ST navigation.) ST navigation is defined similarly to SY navigation except that $\overrightarrow{\operatorname{Tri}}(s, t)(r)$ replaces $\overrightarrow{\mathrm{Cam}}(s, t)(r)$.

\subsection{The random model of stopping places set: a Poisson point process}

Denote by $\operatorname{Lip}^{\star}(\mathscr{D})$ the set of Lipschitz functions $f: \mathscr{D} \rightarrow \mathbb{R}^{+}$with a positive infimum on $\mathscr{D}$ : a function $f$ is in $\operatorname{Lip}^{\star}(\mathscr{D})$ if there exists $\alpha_{f}>0$ such that $|f(x)-f(y)| \leq \alpha_{f}|x-y|$ for all $x, y \in \mathscr{D}$, and $m_{f}:=\inf \{f(x), x \in \mathscr{D}\}>0$. Since $\mathscr{D}$ is bounded, $M_{f}:=\max \{f(x)$, $x \in \mathscr{D}\}<+\infty$.

In this paper we consider a Poisson point process (PPP) $S(f)$ whose intensity measure $\mu_{f}$ on $\mathscr{D}$ is $\mu_{f}(A)=\int_{A} f(z) \mathrm{d} z$ for some $f \in \operatorname{Lip}^{\star}(\mathscr{D})$ (in words, $\mu_{f}$ has density $f$ with respect to the Lebesgue measure on $\mathbb{R}^{2}$ ): the necessity of the Lipschitz condition will be made clear later, with the appearance of a differential equation. The distribution of $\boldsymbol{S}(f)$ is denoted by $\mathbf{P}_{f}$.

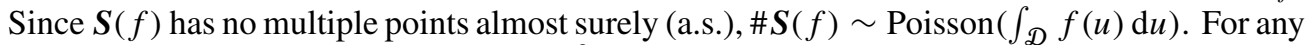
disjoint Borelian subsets $B_{1}, \ldots, B_{k}$ of $\mathbb{R}^{2},\left(S(f) \cap B_{1}\right), \ldots,\left(S(f) \cap B_{k}\right)$ are independent and $\#\left(\boldsymbol{S}(f) \cap B_{j}\right) \sim \operatorname{Poisson}\left(\int_{B_{j}} f(u) \mathrm{d} u\right)$ (for more explanations on PPPs, see Chapter 10 of [12]). As usual, a representation of the set $\boldsymbol{S}(f)$ is $\boldsymbol{S}(f)=\left\{x_{1}, \ldots, x_{\boldsymbol{n}}\right\}$, where $\boldsymbol{n} \sim \operatorname{Poisson}\left(\int_{\mathbb{D}} f\right)$, $x_{1}, x_{2}, \ldots$ are independent and identically distributed (i.i.d.) and independent of $\boldsymbol{n}$, and each $x_{i}$ has density $f / \inf _{\mathscr{D}} f$.

In this paper we assume that the measure $\sum_{x \in S(f)} \delta_{x}$ is defined on a probability space $(\Omega, \mathcal{A}, \mathrm{P})$, and is considered as a random variable taking values in $\mathrm{N}$, the set of counting measures in $\mathbb{C}$, equipped with the $\sigma$-field $\mathcal{N}$ generated by the sets $E_{B, k}=\{\mu, \mu(B)=k\}$ for compact sets $B \subset \mathbb{C}$ and integers $k$.

The model consisting of $n$ i.i.d. points chosen according to a density $f$ is considered in Section 2.3.2.

Remark 2. The navigations $X$ we study, as well as the functions $\operatorname{Path}^{X}$ and $\operatorname{Pos}^{X}$, and various cost functions, are all defined given a stopping places set $S$. Hence, they are functions of $S$ (we should write $\boldsymbol{X}(S)$ etc., but we choose to delete this $S$ to lighten the notation). On $(\Omega, \mathcal{A}, \mathrm{P})$, these functions are random variables with values in some functional spaces. 


\subsection{Quantities of interest}

In (1) we defined $\operatorname{Path}^{X}(s, t)$. In many applications, a quantity of interest is the comparison between the Euclidean distance $|s-t|$ and the path length, the total distance covered by the traveller in the case of success:

$$
\left|\operatorname{Path}^{X}(s, t)\right|:=\sum_{j=1}^{\mathrm{Nb}^{X}(s, t)}\left|s_{j}-s_{j-1}\right| .
$$

The associated trajectory is the compact subset of $\mathbb{R}^{2}$ formed by the union of the segments $\left[s_{j}, s_{j+1}\right]$ :

$$
\left[\operatorname{Path}^{X}(s, t)\right]:=\bigcup_{j=1}^{\mathrm{Nb}^{X}(s, t)}\left[s_{j-1}, s_{j}\right] .
$$

One of the results of the paper is the comparison between $\left[\mathrm{Path}^{X}(s, t)\right]$ and a limiting object.

If the point process is not homogeneous, the evaluation of the traveller's trajectory calls for a precise study of the traveller's speed. We introduce the function $\operatorname{Pos}_{s, t}^{X}:\left[0, \mathrm{Nb}^{X}(s, t)\right] \rightarrow \mathbb{R}^{2}$, whose values coincide with those of $\boldsymbol{X}(s, t, \cdot)$ at integer points, and interpolated inbetween. Hence, $\operatorname{Pos}_{s, t}^{X}$ gives the position of the traveller according to the number of stages; for any $s, t \in \mathcal{D},\left[\right.$ Path $\left.^{X}(s, t)\right]$ equals the range of $\operatorname{Pos}_{s, t}^{X}$. From our point of view, the asymptotic results obtained for $\operatorname{Pos}_{s, t}$ are among the main contributions of this paper.

In some applications, the sum of a function of the stage lengths appears to be the relevant quantity instead of the length. Formally a 'unitary cost function' $H: \mathbb{C} \rightarrow \mathbb{R}$ is considered. The total cost associated with $H$ corresponding to $\operatorname{Path}^{X}(s, j)=\left(s_{0}, \ldots, s_{\mathrm{Nb}^{X}(s, t)}\right)$ is

$$
\operatorname{Cost}_{H}^{X}(s, t):=\sum_{j=1}^{\mathrm{Nb}^{X}(s, t)} H\left(s_{j}-s_{j-1}\right) .
$$

Important elementary cost functions are $H: x \rightarrow|x|$, which gives $\operatorname{Cost}_{H}(s, t)=|\operatorname{Path}(s, t)|$, $H: x \rightarrow 1$, which gives $\operatorname{Cost}_{H}^{X}(s, t)=\mathrm{Nb}^{X}(s, t)$, and functions of the type $H(x)=|x|^{\beta}$, which correspond, at least for some $\beta$, to real applications.

\section{Statements of the main results}

In the rest of this paper, $f$ is a fixed element of $\operatorname{Lip}^{\star}(\mathscr{D})$ and $a$ is a fixed positive number. The set $\mathscr{D}[a]:=\{x \in \mathscr{D}: d(x, \mathfrak{D}) \geq a\}$ is the set of points in $\mathscr{D}$ at distance at least $a$ to $\complement \mathcal{D}$, the complement of $\mathcal{D}$. The asymptotic behaviour of $\operatorname{Path}^{X}(s, t)$ (when the set of stopping places $S$ increases) is difficult to predict if the limiting trajectories between $s$ and $t$ are too close to $C \mathscr{D}$. We set a notation to designate the pairs $(s, t)$ that can be treated. Set

$$
\mathscr{D}^{\prime}[a]:=\left\{(s, t) \in \mathscr{D}^{2}: Z(s, t) \subset \mathscr{D}[a]\right\},
$$

where, according to the context, $Z(s, t)=\Gamma(s, t)$ (for cross navigations) or $Z(s, t)=[s, t]$ (for straight navigations). Some other restrictions concerning $\theta$ will be added in order to avoid situations where the navigation can fail.

Before giving the results, we define some constants, computed in Sections 3.3.1 and 3.3.3, related to the speed of the traveller along their trajectory and the ratio of the mean length of a 
stage to the length of the projection of a stage with respect to some ad-hoc directions:

$$
\begin{aligned}
& \boldsymbol{C}_{\mathrm{bis}}^{\mathrm{ST}}=\boldsymbol{C}_{\mathrm{bis}}^{\mathrm{CT}}=\frac{1}{2} \sqrt{\frac{\pi}{\tan (\theta / 2)}}, \\
& \boldsymbol{C}_{\mathrm{bis}}^{\mathrm{SY}}=\boldsymbol{C}_{\mathrm{bis}}^{\mathrm{CY}}=\frac{\sqrt{2 \pi} \sin (\theta / 2)}{\theta^{3 / 2}}, \\
& C_{\text {bor }}^{\mathrm{CT}}=\sqrt{\frac{\pi \cos ^{3}(\theta / 2)}{4 \sin (\theta / 2)},}, \\
& C_{\text {bor }}^{\mathrm{CY}}=\frac{\sqrt{\pi / 2} \sin (\theta)}{\theta^{3 / 2}}, \\
& \boldsymbol{Q}_{\mathrm{bis}}^{\mathrm{ST}}=\boldsymbol{Q}_{\mathrm{bis}}^{\mathrm{CT}}=\frac{1}{2}\left(\frac{1}{\cos (\theta / 2)}+\frac{\operatorname{arcsinh}(\tan (\theta / 2))}{\tan (\theta / 2)}\right) \text {, } \\
& \boldsymbol{Q}_{\mathrm{bis}}^{\mathrm{SY}}=\boldsymbol{Q}_{\mathrm{bis}}^{\mathrm{CY}}=\frac{\theta / 2}{\sin (\theta / 2)}, \\
& \boldsymbol{Q}_{\mathrm{bor}}^{\mathrm{CT}}=\frac{1}{2}\left(\frac{1}{\cos ^{2}(\theta / 2)}+\frac{\operatorname{arcsinh}(\tan (\theta / 2))}{\sin (\theta / 2)}\right) \text {, } \\
& \boldsymbol{Q}_{\text {bor }}^{\mathrm{CY}}=\frac{\theta}{\sin (\theta)} \text {. }
\end{aligned}
$$

\subsection{Limit theorems for straight navigations}

The first theorem uniformly compares the path length $\left|\operatorname{Path}^{X}(s, t)\right|$ with a multiple of $|s-t|$.

Theorem 1. Let $\boldsymbol{X}=\mathrm{SY}$ and $\theta<\pi / 2$, or $\boldsymbol{X}=\mathrm{ST}$ and $\theta \leq \pi / 2$. For any $\alpha \in\left(0, \frac{1}{8}\right)$, any $\beta>0$, and large enough $n$,

$$
\mathrm{P}_{n f}\left(\sup _{(s, t) \in \mathscr{D}^{\prime}[a]}|| \operatorname{Path}^{X}(s, t)\left|-Q_{\text {bis }}^{X} \times\right| s-t|| \geq n^{-\alpha}\right) \leq n^{-\beta} .
$$

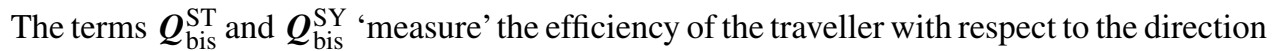
of the bisecting lines of the decision sectors used.

We now describe the asymptotic behaviour of $\operatorname{Pos}_{s, t}^{X}$. In the case of straight navigation, the limiting position function $\operatorname{Pos}^{\infty, c}$ is the deterministic solution of a differential equation, and depends on a real parameter $c$, where $c$ depends on $\theta$ and $\boldsymbol{X}$.

For any $(\lambda, v) \in(0,+\infty) \times[0,2 \pi]$, let $F_{\lambda, v}$ be the function from $\mathscr{D}$ to $\mathbb{C}$ defined by

$$
F_{\lambda, v}(z):=\frac{\lambda \mathrm{e}^{\mathrm{i} v}}{\sqrt{f(z)}} .
$$

For given $s_{0} \in \mathscr{D}$, consider the following ordinary differential equation (ODE):

$$
\operatorname{ODE}\left(\lambda, v, s_{0}\right):=\left\{\begin{array}{l}
\rho(0)=s_{0}, \\
\frac{\partial \rho(x)}{\partial x}=F_{\lambda, v}(\rho(x)) .
\end{array}\right.
$$

By the Cauchy-Lipschitz theorem, $\operatorname{ODE}\left(\lambda, \nu, s_{0}\right)$ admits a unique solution $x \mapsto \operatorname{Sol}_{s_{0}}^{\lambda, v}(x)$ valid for $x \geq 0$ and while $\operatorname{Sol}_{s_{0}}^{\lambda, v}(x)$ stays inside $\mathscr{D}$; indeed, since $f$ is in $\operatorname{Lip}^{\star}(\mathscr{D})$, so is $F_{\lambda, v}$. Since $f$ takes its values in $\mathbb{R}^{+}, \arg \left(F_{\lambda, v}\right)=v$. Hence, $\operatorname{Sol}_{s_{0}}^{\lambda, v}$ appears to be a monotone parametrisation of the segment $\left\{s_{0}+x \mathrm{e}^{\mathrm{i} v}, x>0\right\} \cap \mathscr{D}$ (more precisely, of the component containing $s_{0}$ in this intersection), with the speed along this segment at position $z$ being $F_{\lambda, v}(z)$. Now, choose two points $(s, t)$ in $\mathscr{D}$ such that $[s, t] \subset \mathscr{D}$ and consider $\operatorname{Sol}_{s}^{\lambda, \arg (t-s)}$ for some fixed $\lambda>0$. In this case, the range of $\operatorname{Sol}_{s}^{\lambda, \arg (t-s)}$ contains $[s, t]$ and, by continuity, there exists a time Time ${ }_{s, t}^{\lambda}$ such that

$$
\operatorname{Sol}_{s}^{\lambda, \arg (t-s)}\left(\operatorname{Time}_{s, t}^{\lambda}\right)=t .
$$

We now define the function $\operatorname{Pos}_{s, t}^{\infty, \lambda}$ as the function $\operatorname{Sol}_{s}^{\lambda, \arg (t-s)}$ stopped at $t$. 
Theorem 2. Let $\boldsymbol{X}=\mathrm{SY}$ and $\theta<\pi / 2$, or $\boldsymbol{X}=\mathrm{ST}$ and $\theta \leq \pi / 2$. For any $\alpha \in\left(0, \frac{1}{8}\right)$, any $\beta>0$, and large enough $n$,

$$
\mathrm{P}_{n f}\left(\sup _{(s, t) \in D^{\prime}[a]} \sup _{x \geq 0}\left|\operatorname{Pos}_{s, t}^{\boldsymbol{X}}(x \sqrt{n})-\operatorname{Pos}_{s, t}^{\infty, C_{\text {bis }}^{X}}(x)\right| \geq n^{-\alpha}\right) \leq n^{-\beta} .
$$

A corollary of this theorem is that the asymptotic trajectories of the traveller are segments: for $d_{H}$, the Hausdorff distance between the compact subset of $\mathbb{R}^{2}, \mathrm{P}_{n f}\left(\sup _{(s, t) \in \mathcal{D}^{\prime}[a]} d_{H}([\operatorname{Path}(s, t)\right.$, $\left.[s, t]) \geq n^{-\alpha}\right) \leq n^{-\beta}$.

The asymptotic behaviour of different cost functions is studied in Section 4.2.

\subsection{Limit theorems for cross navigation}

For any $c_{1}>0, c_{2}>0$, and any $s, t \in \mathbb{C}$, let

$$
\boldsymbol{D}_{c_{1}, c_{2}}(s, t):=c_{1} \times|I(s, t)-s|+c_{2} \times|t-I(s, t)|
$$

be a kind of weighted length of $\Gamma(s, t)$.

The cross navigation analogy of Theorem 1 is as follows (see Figure 5).

Theorem 3. Let $\boldsymbol{X} \in\{\mathrm{CT}, \mathrm{CY}\}$ and $\theta \leq \pi / 3$. For any $\alpha \in\left(0, \frac{1}{8}\right)$, any $\beta>0$, and large enough $n$,

$$
\mathrm{P}_{n f}\left(\sup _{(s, t) \in D^{\prime}[a]}|| \operatorname{Path}^{X}(s, t)\left|-\boldsymbol{D}_{\boldsymbol{Q}_{\text {bis }}^{X}, \boldsymbol{Q}_{\text {bor }}^{X}}(s, t)\right| \geq n^{-\alpha}\right) \leq n^{-\beta} .
$$

The terms $\boldsymbol{Q}_{\mathrm{bor}}^{\mathrm{ST}}$ and $\boldsymbol{Q}_{\mathrm{bor}}^{\mathrm{SY}}$ measure some local efficiency of the traveller with respect to the direction of the border of the decision sectors.

As mentioned above, CY is the canonical navigation on Yao's graph, and CT is the canonical navigation on the so-called $\Theta$-graphs [10], [13], [18]. A worst case study has been made showing that the stretch factor of these navigations is at most $1 /\left(1-2 \sin \pi / p_{\theta}\right)$ for every $p_{\theta}>6$. For $p_{\theta} \leq 6$, the stretch factor of these navigations can be unbounded. However, it has been proved that $\Theta_{6}$ is a 2-spanner [7], Yao 6 is a $c$-spanner (for some $c$ ) [15], and $\mathrm{Yao}_{4}$ is an $8(29+23 \sqrt{2})$-spanner [9]. The theorem obtained here says that 'for a typical set of points', the navigation distance, and also the graph distance, between any two points $s$ and $t$ is smaller than $\boldsymbol{D}_{\boldsymbol{Q}_{\text {bis }}^{X}, \boldsymbol{Q}_{\text {bor }}^{X}}(s, t)+n^{-\alpha}$ with large probability. For far away points $s$ and $t$, this is much smaller than the worst case bounds.

Again, for $X \in\{\mathrm{CT}, \mathrm{CY}\}$, the function $x \mapsto \operatorname{Pos}_{s, t}^{X}(x \sqrt{n})$ admits a deterministic limit $\operatorname{Pos}_{s, t}^{\infty, a, b}$ depending on the parameters $a$ and $b$ (which depend on $\boldsymbol{X}$ and $\theta$ ) related to the speed

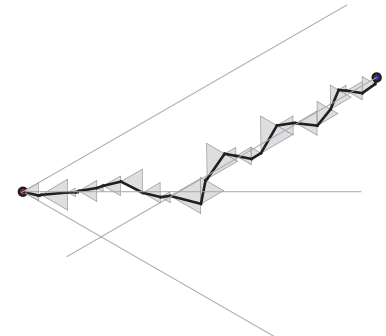

(a)

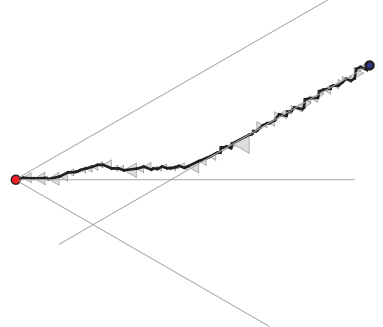

(b)

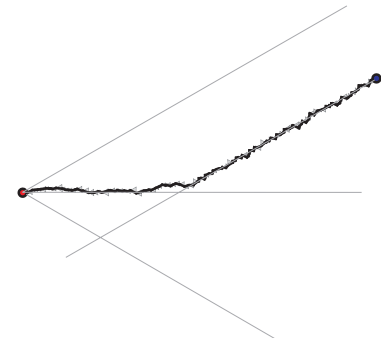

(c)

Figure 5: Examples of $p_{\theta}$-CT navigation (for $p_{\theta}=6$ ) with (a) 500 nodes, (b) 5000 nodes, and (c) 20000 nodes uniformly distributed in the unit square. 
of the traveller along the two branches of $\Gamma(s, t)$. Set

$$
\operatorname{Pos}_{s, t}^{\infty, \lambda, \lambda^{\prime}}(x):= \begin{cases}\operatorname{Sol}_{s}^{\lambda, \arg (I(s, t)-s)}(x) & \text { for } x \in\left[0, \operatorname{Time}_{s, I(s, t)}^{\lambda}\right], \\ \operatorname{Sol}_{I(s, t)}^{\lambda^{\prime}, \arg (t-I(s, t))}\left(x-\operatorname{Time}_{s, I(s, t)}^{\lambda(1)}\right) & \text { if } x \in \operatorname{Time}_{s, I(s, t)}^{\lambda}+\left[0, \operatorname{Time}_{I(s, t), t}^{\lambda^{\prime}}\right], \\ t & \text { if } x \geq \operatorname{Time}_{s, I(s, t)}^{\lambda\left(\operatorname{Time}_{I(s, t), t}^{\lambda^{\prime}}\right.} .\end{cases}
$$

Theorem 4. Let $\boldsymbol{X} \in\{\mathrm{CY}, \mathrm{CT}\}$ and $\theta \leq \pi / 3$. For any $\alpha \in\left(0, \frac{1}{8}\right)$, any $\beta>0$, and large enough $n$,

$$
\mathrm{P}_{n f}\left(\sup _{(s, t) \in \mathscr{D}^{\prime}[a]} \sup _{x \geq 0}\left|\operatorname{Pos}_{s, t}^{X}(x \sqrt{n})-\operatorname{Pos}_{s, t}^{\infty, C_{\text {bis }}^{X}, C_{\text {bor }}^{X}}(x)\right| \geq n^{-\alpha}\right) \leq n^{-\beta} .
$$

As for the straight case, this entails the convergence of the Hausdorff distance from Path $(s, t)$ to $\Gamma(s, t)$.

Again, results concerning other cost functions are studied in Section 4.2.

\subsection{Extensions}

The following extensions can be treated with the material available in the present paper. We provide just the main lines of analysis.

2.3.1. Random north navigation. This is a version of cross navigation where each point $s \in S$ has its own (random) north $n(s)$ used to compute Cross $(s)$. This can be used to model some imprecisions in Yao's construction, where the north is not exactly known by the points of $S$. Each distribution of $n(s)$ leads to a different behaviour for the traveller. If the value of $n(s)$ is 0 a.s. then this model coincides with standard cross navigation. If the distribution of $n(s)$ owns several atoms then the speed of the traveller may be different along numerous directions. The corresponding navigation algorithm is defined as follows. A traveller at $s$ chooses the smallest integer $\kappa$ in $\llbracket 0, p_{\theta}-1 \rrbracket$ such that $t$ lies in $s+\mathrm{e}^{\mathrm{i} n(s)+\mathrm{i} k \theta} \operatorname{Sect}(\theta)$ and considers the smallest $r$ such that $s+\mathrm{e}^{\mathrm{i} n(s)+\mathrm{i} k \theta} \operatorname{Tri}(\theta)(r) \cap(S \cup\{t\})$ is not empty. Then set $\operatorname{RNT}(s, t)=z$, the element of this set with smallest argument.

From now on, the random variables $(n(s), s \in S)$ are assumed to be i.i.d. and to take their values in $[0,2 \pi]$. Using the Camembert sections instead leads to the random north Yao navigation, abbreviated as RNY. Uniform random north navigation is not much different from straight navigation, with the limiting path being segments and the limiting path length being a multiple of the Euclidean distance. Using the same arguments as above, we can prove that the distance covered by the traveller satisfies, for any $\theta \leq \pi / 3$, any $\alpha \in\left(0, \frac{1}{8}\right)$, and $\beta>0$, if $n$ is large enough,

$$
\mathrm{P}_{n f}\left(\sup _{(s, t) \in \mathscr{D}^{\prime}[a]}\left|\operatorname{Path}^{X}(s, t)-Q^{X}\right| s-t|| \geq n^{-\alpha}\right) \leq n^{-\beta},
$$

where

$$
\boldsymbol{Q}^{\mathrm{RNT}}=\frac{\theta / 2}{2 \sin (\theta / 2)}\left(\frac{1}{\cos (\theta / 2)}+\frac{\operatorname{arcsinh}(\tan (\theta / 2))}{\tan (\theta / 2)}\right), \quad \boldsymbol{Q}^{\mathrm{RNY}}=\frac{\theta^{2}}{2-2 \cos (\theta)} .
$$

The computation of these constants is carried out in Section 3.3.4; the proof of the globalisation of the bounds does not present any problem in this case. 
2.3.2. Model of $n$ i.i.d. positions. Consider $g \in \operatorname{Lip}^{\star}(\mathscr{D})$ such that $\mathbf{1}_{\mathscr{D}} g=1$. Let $p_{1}, \ldots, p_{n}$ be $n$ i.i.d. random points chosen in $\mathcal{D}$ under the distribution with density $g$. Set $S_{g}^{n}:=\left\{p_{1}, \ldots, p_{n}\right\}$, and denote by $\operatorname{Pr}_{g}^{n}$ the distribution of this set. The analysis of the navigations under $\mathrm{P}_{n g}$ is simpler than under $\operatorname{Pr}_{g}^{n}$ since under $\mathrm{P}_{n g}$ Markovian properties can be used; the derivation of the results under $\operatorname{Pr}_{g}^{n}$ will appear to be simple consequences of those under $\mathrm{P}_{n g}$. Indeed, the two models $\operatorname{Pr}_{g}^{n}$ and $\mathrm{P}_{n g}$ are related via the classical fact

$$
\operatorname{Pr}_{g}^{n}(\cdot)=\mathrm{P}_{n g}(\cdot \mid \# \boldsymbol{S}=n) ;
$$

in other words $\boldsymbol{K}_{n g}$ conditioned by $\# \boldsymbol{S}(n g)=n$ has the same distribution as $\boldsymbol{K}_{g}^{n}$.

For any measurable event $A$ (element of $\mathcal{N}$ as defined in Section 1.3) and any $c>0$,

$$
\operatorname{Pr}_{g}^{n}(A)=\mathrm{P}_{n g}(A \mid \# \boldsymbol{S}=n) \leq \frac{\mathrm{P}_{n g}(A)}{\mathrm{P}_{n g}(\# \boldsymbol{S}=n)} .
$$

Since $\# \boldsymbol{S}(n g)$ is Poisson $(n)$ distributed, we see that $\mathrm{P}_{n g}(\# \boldsymbol{S}=n) \geq c_{1} n^{-1 / 2}$ for large enough $n$ and a constant $c_{1}>0$ (this is an application of the Stirling formula); therefore,

$$
\operatorname{Pr}_{g}^{n}(A) \leq \frac{1}{c_{1}} n^{1 / 2} \mathrm{P}_{n g}(A)
$$

for large enough $n$. All the results of the present paper under the form $\mathrm{P}_{n g}\left(A_{n}\right)=o\left(x_{n}\right)$ for some decreasing function $x_{n}$ can be transferred under $\operatorname{Pr}_{g}^{n}$ to the form $\operatorname{Pr}_{g}^{n}\left(A_{n}\right)=O\left(n^{1 / 2} \mathrm{P}_{n g}\left(A_{n g}\right)\right)=$ $o\left(n^{1 / 2} x_{n}\right)$, that is, a factor $n^{1 / 2}$ on the bound must be added. Therefore, the main theorems of the present paper can be transferred to $\operatorname{Pr}_{g}^{n}$ without any problems since all results (or intermediate results) have the form $\mathrm{P}_{n g}\left(A_{n}\right)=o\left(x_{n}\right)$ for $x_{n}=o\left(n^{-1 / 2}\right)$.

\subsection{Link with other random objects}

In [5], the authors investigated a navigation $N$ on a homogeneous PPP $S$ on $\mathbb{C}$, where the traveller wants to reach the origin 0 of the plane: for a given $s, N(s)$ is the closest element of $s$ in $S \cup\{0\}$, which has the additional property of being closer to 0 than $s$. Adding an edge between each $s \in S$ and $N(s)$ gives a tree, called the 'radial spanning tree' of the PPP. (See the straight $p_{\theta}$-Yao navigation with $\theta=2 \pi / 3$ in Figure $6(\mathrm{~b})$; this picture is quite similar to Figure 1 of [5] even if the navigation is less homogeneous in that paper.) The authors provided numerous results concerning this tree, including some local properties concerning the degree

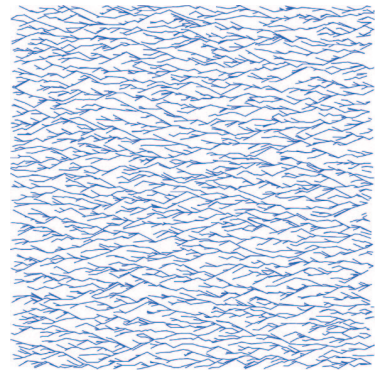

(a)

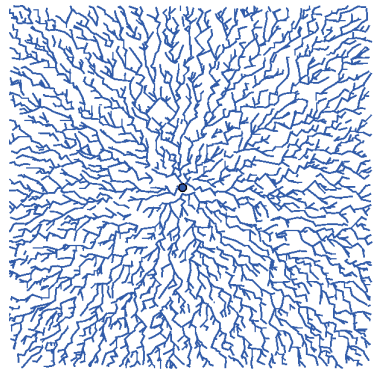

(b)

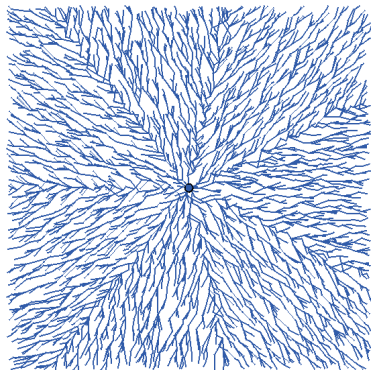

(c)

FIGURE 6: Union of all paths (toward a unique target) on 5000 uniform random points for (a) a directed navigation with $\theta=2 \pi / 7$, (b) an SY navigation with $\theta=2 \pi / 3$, and (c) a CT navigation with $p_{\theta}=7$. 
of the vertices, the length of the edges, and some global properties, such as the behaviour of the subset of elements $s$ of $S$ such that $N(s, k)=0$. Bordenave [8] also examined the radial spanning tree of the PPP. He studied functionals along a path, and topological issues such as the directions of the paths going to $\infty$, and showed that the number of ends of the tree is infinite.

In [6] and [16] the authors studied the property of a so-called 'minimal directed spanning tree' (MDST). To each finite subset $S$ of $(0,1]^{2}$ is associated a tree as follows: $x \in S$ is connected by a directed edge to the nearest $y \in S \cup\{(0,0)\}$, having both coordinates smaller than those of $x$. They then studied the asymptotic total length of the MDST when $S$ follows various distributions, with $|S| \rightarrow+\infty$.

In [4], the authors examined a directed-like navigation, where the set of points is a random subset of $\mathbb{Z}^{d}$ : each point of this set is kept with probability $p \in(0,1)$. They then examined the connectivity of the construction according to the dimension.

Another object that may be related to directed navigation is the Brownian web. In [11], the authors constructed a binary tree using as a set of vertices the points of a homogeneous PPP in the plane. The parent-children relation is then induced by a kind of navigation, where, instead of a Camembert section, a rectangular section is used; this is similar to what is represented in Figure 6(a). They then showed that in a certain sense their object converges to the Brownian web (see the references therein for definitions and criteria of convergences to the Brownian web). In the present paper we conjecture that the work in [11] can be extended to Yao graphs or $\Theta$-graphs associated to directed navigations. The main structural difference here is that the infinite tree constructed through directed navigation has an unbounded degree, whereas in [11] it has a binary degree.

In a sequence of papers, Aldous and coauthors [1], [2], [3] investigated numerous questions related to navigation (or traveller salesman-type problems) in a PPP. In particular, Aldous [1] gave sufficient conditions on navigation algorithms to have an asymptotic shape (where the shape is roughly the set of points at a distance smaller than $r$ to the origin, properly rescaled).

\section{Theoretical considerations}

\subsection{Presentation of the analysis}

We present here the main ideas used in the paper before entering into the details.

- Under $\mathrm{P}_{n f}$, a ball of radius $r$ included in $\mathscr{D}$ contains $O\left(n r^{2}\right)$ points of $\boldsymbol{S}(n f)$ on average and, with large probability, less than $n^{1+\varepsilon} r^{2}$, uniformly on all balls (see Lemma 3). We restrict ourselves to the case where the navigation has the property to force the traveller to come closer to $t$ at each stage. Hence, for a radius $r=r_{n}$ small enough, Lemma 3 allows us to show that the contribution of the stages of the traveller in the final ball of radius $r$ is negligible. See Section 3.5.

- To study the behaviour of the traveller far from its target, a local argument is used: under $\mathrm{P}_{n f}$ for a nonconstant function $f$, the stages $\left(s_{j}-s_{j-1}, j \geq 1\right)$ are not identically distributed, or independent since the value of $s_{j-1}$ affects the distribution of $s_{j}-s_{j-1}$. But, if we consider only $a_{n}$ successive stages, with $a_{n} \rightarrow+\infty$ and $a_{n} n^{\varepsilon} / \sqrt{n} \rightarrow 0$, these stages stay in a small window around $s_{0}$ with a large probability; these stages appear moreover to roughly behave as i.i.d. random variables under the homogeneous PPP $\mathrm{P}_{n f\left(s_{0}\right)}$; the behavior of these $a_{n}$ stages are seen to depend, at the first order, only on $f\left(s_{0}\right)$. Moreover, since $a_{n} \rightarrow+\infty$, some regularizations of the type 'law of large numbers' occur. See Section 3.3. This local theorem is one of the cornerstones of the 
study: in some sense, the global trajectory between any two points is a concatenation of these parts of length $a_{n}$; the successive local values $\left(f\left(s_{k \times a_{n}}\right), k \geq 0\right)$ directly lead to a differential equation. See Section 3.4.

- The speed of convergence stated in the theorems is roughly given for each part of length $a_{n}$ : it is shown that well-chosen deviations are exponentially rare, and that the deviation between an entire trajectory and the limiting solution of the ODE has exponentially rare deviations. Hence, without extra effort, this result may be extended to all trajectories starting and ending on the two-dimensional grid $n^{-w} \mathbb{Z}^{2} \cap \mathcal{D}$ for some $w>0$. The final globalisation consists in comparing the paths between any two points $s$ and $t$ of $\mathscr{D}$, and some well-chosen points of the grid $n^{-w} \mathbb{Z}^{2} \cap \mathcal{D}$. This is possible if $w$ is large enough. See Section 4.3.

\subsection{The termination of navigations}

The main aim of this section is to state and prove the following proposition.

Proposition 1. Let $\boldsymbol{X}=\mathrm{SY}$ and $\theta \leq 2 \pi / 3$, or $\boldsymbol{X}=\mathrm{ST}$ and $\theta \leq \pi / 2$, or $\boldsymbol{X} \in\{\mathrm{CT}, \mathrm{CY}\}$ and $\theta \leq \pi / 3$. Under $\mathrm{P}_{n f}$, a.s. for all $(s, t) \in \mathscr{D}^{\prime}[a]$,

(i) the navigation from $s$ to $t$ succeeds (i.e. there exists a $k$ such that $\boldsymbol{X}(s, t, k)=t$ );

(ii) the traveller comes closer to the target at each step (i.e. $\left.\operatorname{Path}^{X}(s, t) \subset B(t,|s-t|)\right)$;

(iii) $\left|\operatorname{Path}^{X}(s, t)\right| \leq 2|s-t| \times \# S \cap B(t,|s-t|)$;

(iv) if $\left|s_{1}-s\right| \leq|t-s| / 2$ then $|t-s|-\left|t-s_{1}\right| \geq(2-\sqrt{3})\left|s_{1}-s\right|$.

Proof. Let $\kappa$ be an integer such that $t \in \operatorname{Sect}[\kappa, s]$, and let $t_{\kappa}$ be the orthogonal projection of $t$ on the bisecting line of the sector $\kappa$. Let Area be $\operatorname{Cam}[\kappa, s](|s-t|)$, Tri $[\kappa, s]\left(\left|s-t_{\kappa}\right|\right)$, $\overrightarrow{\mathrm{Cam}}(s, t)(|s-t|)$, or $\overrightarrow{\mathrm{Tri}}(s, t)(|s-t|)$ if $\boldsymbol{X}$ is CY, CT, SY, or ST, respectively. For the values of $\theta$ considered, Area $\subset B(t,|s-t|)$. In each case, $s_{1} \in$ Area, and so $\left|s_{1}-t\right| \leq|s-t|$, which implies part (ii) of the proposition. The previous inequality is strict except in one particular case for each navigation considered and for only the maximal values of $\theta$ considered, i.e. in the case Area contains the two points $t$ and $s_{1}$ only, and $s_{1}$ is on the first border of Area such that $\left|s_{1}-t\right|=|s-t|$. Observe that in this case, $\arg \left(s_{1}-t\right)-\arg (s-t)$ equals $\pi / 2$ if $X=\mathrm{ST}$ and $\pi / 3$ for the other navigations (still for the maximal values of $\theta$ considered). Hence, the navigation fails only if there exists $(s, t) \in \mathscr{D}^{2}$ such that $S \cup\{s\}$ contains four points or six points forming an empty square or an empty hexagon, respectively, centred at $t$ with no other points of $S$ inside this polygon. Under $\mathrm{P}_{n f}$, a.s., $S$ does not contain such a configuration. This implies part (i) of the proposition.

Part (iii) follows directly from parts (i) and (ii): the length of each stage of $\operatorname{Path}^{X}(s, t)$ is at most $2|s-t|$ and $\operatorname{Path}^{X}(s, t)$ is composed of at most $\# S \cap B(t,|s-t|)$ stages.

Now let us prove part (iv). Let $\alpha:=\arg (t-s)-\arg \left(s_{1}-s\right)$. For the navigation considered, $\alpha \in[0, \pi / 3]$. By the cosine law we have

$$
\left|t-s_{1}\right|^{2}=|t-s|^{2}+\left|s_{1}-s\right|^{2}-2|t-s|\left|s_{1}-s\right| \cos \alpha .
$$

Let $x \geq 2$ such that $|t-s|=x\left|s_{1}-s\right|$. By (3), $\left|t-s_{1}\right|=\left|s_{1}-s\right| \sqrt{x^{2}+1-2 x \cos \alpha}$ and then $|t-s|-\left|t-s_{1}\right|=\left|s_{1}-s\right|\left(x-\sqrt{x^{2}+1-2 x \cos \alpha}\right)$. For $x \geq 2$, using the fact that $\alpha \mapsto$ $\cos \alpha$ is decreasing on $[0, \pi / 3], x-\left(x^{2}+1-x\right)^{1 / 2} \leq x-\sqrt{x^{2}+1-2 x \cos \alpha} \leq x-(x-1)$. Therefore, $\left(x-\sqrt{x^{2}+1-2 x \cos \alpha}\right) \in[2-\sqrt{3}, 1]$, completing the proof of part (iv). 


\subsection{A notion of directed navigation}

In the straight navigation under $\mathrm{P}_{n f}$, when the traveller is far from their target, the fluctuations of $\arg \left(t-s_{i}\right)$ stay small for the first values of $i$ (for large $n$ ). It is then intuitive that the trajectory of the traveller would not be much changed if instead of using $\arg \left(t-s_{i}\right)$ the constant direction given by $\arg \left(t-s_{0}\right)$ had been used; similarly, in the cross navigation, when the traveller is far from Cross $(t)$, it is easily seen that along their first stages the bisecting lines of their decision sectors are parallel to the bisecting line of the first decision sector, in other words, the traveller follows the direction of the bisecting line of the sector around $s_{0}$ containing $t$. In order to make these phenomena clear, the directed navigation (DT navigation) and directed Yao navigation (DY navigation) are defined below.

Again $\theta$ is a fixed parameter chosen in $(0,2 \pi)$.

Definition 5. (DT navigation.) DT navigation is a map from $\mathbb{C}$ to $S$ defined as follows. Let $s \in \mathbb{C}$. If $\overrightarrow{\operatorname{Sect}}(s,+\infty) \cap S$ is empty then DT $(s)$ is defined to be $s$. Otherwise, consider the smallest $r$ such that $(s+\operatorname{Tri}(\theta)(r)) \cap S$ is not empty. Then DT $(s)$ is defined to be the element of this intersection, that is, the closest to the first border of $\overrightarrow{\operatorname{Sect}}(s,+\infty)$.

Definition 6. (DY navigation.) DY navigation is defined similarly to DT navigation except that $(s+\operatorname{Cam}(\theta)(r)) \cap S$ replaces $(s+\operatorname{Tri}(\theta)(r)) \cap S$.

For any $s \in \mathbb{C}$, the successive stopping places (DT $(s, j), j \geq 0$ ) of the traveller satisfy $\mathrm{DT}(s, 0)=s, \mathrm{DT}(s, j)=\mathrm{DT}(\mathrm{DT}(s, j-1))$. Informally, DT navigation coincides with ST navigation if the target is $t=+\infty$. If the directed navigation is done on a homogeneous PPP on $\mathbb{C}$, the stages

$$
\Delta^{\mathrm{DT}}(s, j):=\mathrm{DT}(s, j)-\mathrm{DT}(s, j-1), \quad j \geq 1,
$$

are i.i.d. Under $\mathrm{P}_{f}$, for a nonconstant $f$, the process (DT( $\left.s, j\right), j \geq 0$ ) is Markovian, but the stages are not i.i.d. (see the asymptotic behaviour of the directed navigation in Figure 6(a)).

3.3.1. Directed navigation on a PPP with constant intensity. We now consider DT navigation under $\mathrm{P}_{c}$ on all $\mathbb{C}$. Here the subscript $c$ on $\mathrm{P}$ is used to more clearly indicate the rescaling arguments. Note further that $\Delta^{\mathrm{DT}}(s, j)$ does not depend on $s$; thus, we omit it from the notation. We also remove the superscript DT. For any $j \geq 1$, write

$$
\Delta_{c}(j)=x_{c}(j)+\mathrm{i} y_{c}(j) \text { for } x_{c}(j), y_{c}(j) \in \mathbb{R},
$$

and set $l_{c}(j)=\left|\Delta_{c}(j)\right|$, the length of this stage. The stages $\left(\Delta_{c}(j), j \geq 1\right)$ are i.i.d., as well as the lengths $\left(l_{c}(j), j \geq 1\right)$ and the pairs $\left(\left(x_{c}(j), y_{c}(j)\right), j \geq 1\right)$. By an obvious space rescaling argument, we have the following equalities in distribution for any $j \geq 1$ :

$$
\Delta_{c}(j) \stackrel{\mathrm{D}}{=} \frac{1}{\sqrt{c}} \Delta_{1}(j), \quad\left(x_{c}(j), y_{c}(j)\right) \stackrel{\mathrm{D}}{=} \frac{1}{\sqrt{c}}\left(x_{1}(j), y_{1}(j)\right), \quad l_{c}(j) \stackrel{\mathrm{D}}{=} \frac{1}{\sqrt{c}} l_{1}(j) .
$$

In what follows, we write $x_{1}$ instead of $x_{1}(1), y_{1}$ instead of $y_{1}(1)$, etc. For any $r>0$,

$$
\mathrm{P}\left(x_{1}>r\right)=\mathrm{P}_{1}(\# \operatorname{Tri}[\kappa, 0](r) \cap S=0)=\exp \left(-r^{2} \tan \left(\frac{1}{2} \theta\right)\right),
$$

and so, by integration, we find that $\mathrm{E}\left(x_{1}^{\mathrm{DT}}\right)=\frac{1}{2} \sqrt{\pi / \tan (\theta / 2)}$. Note also that $\mathrm{E}\left(x_{1}^{\mathrm{DT}}\right)=\boldsymbol{C}_{\mathrm{bis}}^{\mathrm{ST}}=$ $\boldsymbol{C}_{\text {bis }}^{\mathrm{CT}}$. Now, since $y_{1}=x_{1} U$, where $U$ is independent of $x_{1}$ and uniform on $[-\tan (\theta / 2), \tan (\theta / 2)]$,

$$
l_{1}=\left|\Delta_{1}\right|=\sqrt{x_{1}^{2}+y_{1}^{2}}=x_{1}\left(1+\tan ^{2}\left(\frac{1}{2} \theta\right) V^{2}\right)^{1 / 2}
$$




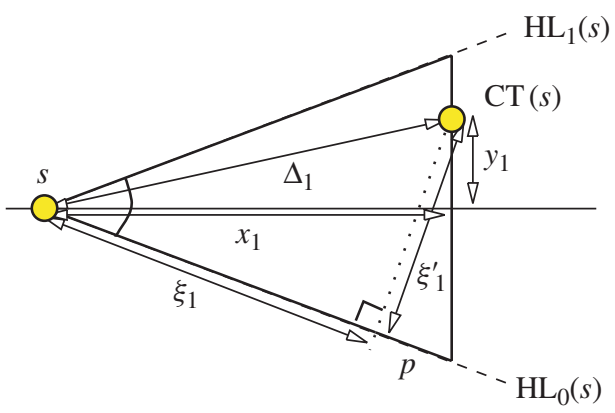

FIGURE 7: Representations of $\xi_{1}$ and $\xi_{1}^{\prime}$.

for $V$ uniform in [0, 1], independent of $x_{1}$. A trite computation leads to $\mathrm{E}\left(\sqrt{1+\tan ^{2}(\theta / 2) V^{2}}\right)=$ $\boldsymbol{Q}_{\text {bis }}^{\mathrm{ST}}$, as given in the beginning of Section 2 , and then $\mathrm{E}\left(l_{1}^{\mathrm{DT}}\right)=\mathrm{E}\left(x_{1}^{\mathrm{DT}}\right) \boldsymbol{Q}_{\mathrm{bis}}^{\mathrm{ST}}=\boldsymbol{Q}_{\mathrm{bis}}^{\mathrm{ST}} \boldsymbol{C}_{\text {bis }}^{\mathrm{ST}}$. We consider another quantity that will play a special role during the analysis of cross navigation. Consider the coordinates $\left(\xi_{1}, \xi_{1}^{\prime}\right)$ of a stage $\Delta_{1}$ in the coordinate system $\left(0, \mathrm{e}^{-\mathrm{i} \theta / 2}, \mathrm{e}^{-\mathrm{i} \theta / 2+\mathrm{i} \pi / 2}\right)$ :

$$
\Delta_{1}=\xi_{1} \mathrm{e}^{-\mathrm{i} \theta / 2}+\xi_{1}^{\prime} \mathrm{e}^{-\mathrm{i} \theta / 2+\mathrm{i} \pi / 2} .
$$

As seen in Figure 7, $\xi_{1}$ is the length of the projection of $\Delta_{1}$ on $\operatorname{HL}_{0}(s)$. The middle of the projections of $\left(x_{1}, y_{1}\right)$ and $\left(x_{1},-y_{1}\right)$ coincides with the projection of $\left(x_{1}, 0\right)$, and, by symmetry of the law of $y_{1}$,

$$
\mathrm{E}\left(\xi_{1}^{\mathrm{DT}}\right)=\mathrm{E}\left(x_{1}^{\mathrm{DT}}\right) \cos \left(\frac{1}{2} \theta\right)=\boldsymbol{C}_{\text {bor }}^{\mathrm{CT}} .
$$

Now consider the distance covered and the position of the traveller after $k$ stops:

$$
\begin{aligned}
X_{1}^{\mathrm{DT}}(k) & =\sum_{j=1}^{k} x_{1}^{\mathrm{DT}}(j), & Y_{1}^{\mathrm{DT}}(k) & =\sum_{j=1}^{k} y_{1}^{\mathrm{DT}}(j), \\
L_{1}^{\mathrm{DT}}(k) & =\sum_{j=1}^{k} l_{1}^{\mathrm{DT}}(j), & \Xi_{1}^{\mathrm{DT}}(k) & =\sum_{j=1}^{k} \xi_{1}^{\mathrm{DT}}(j) .
\end{aligned}
$$

By the law of large numbers, the following 4-tuple converges a.s.:

$$
\left(\frac{X_{1}^{\mathrm{DT}}(k)}{k}, \frac{Y_{1}^{\mathrm{DT}}(k)}{k}, \frac{L_{1}^{\mathrm{DT}}(k)}{k}, \frac{\Xi_{1}^{\mathrm{DT}}(k)}{k}\right) \underset{k}{\stackrel{\text { a.s. }}{\longrightarrow}}\left(\boldsymbol{C}_{\text {bis }}^{\mathrm{CT}}, 0, \mathrm{E}\left(l_{1}^{\mathrm{DT}}\right), \boldsymbol{C}_{\mathrm{bor}}^{\mathrm{CT}}\right) .
$$

Then we also have

$$
\frac{L_{1}^{\mathrm{DT}}(k)}{X_{1}^{\mathrm{DT}}(k)} \underset{k}{\stackrel{\text { a.s. }}{\longrightarrow}} \frac{\mathrm{E}\left(l_{1}^{\mathrm{DT}}\right)}{\mathrm{E}\left(x_{1}^{\mathrm{DT}}\right)}=\boldsymbol{Q}_{\text {bis }}^{\mathrm{ST}} \quad \text { and } \quad \frac{\Xi_{1}^{\mathrm{DT}}(k)}{X_{1}^{\mathrm{DT}}(k)} \underset{k}{\stackrel{\text { a.s. }}{\longrightarrow}} \frac{\mathrm{E}\left(l_{1}^{\mathrm{DT}}\right)}{\mathrm{E}\left(\xi_{1}^{\mathrm{DT}}\right)}=\boldsymbol{Q}_{\text {bor }}^{\mathrm{CT}} .
$$

These numbers are the crucial coefficients appearing in Theorems 1 and 3.

3.3.2. Control of the fluctuations of $X_{1}^{\mathrm{DT}}(k), Y_{1}^{\mathrm{DT}}(k)$, and $L_{1}^{\mathrm{DT}}(k)$. First, it is easily checked that the random variables $l_{1}, x_{1}, y_{1}$, and $\xi_{1}$ have exponential moments. Indeed, by $(5), \mathrm{E}\left(\mathrm{e}^{t\left|x_{1}\right|}\right)<$ $+\infty$ for any $t>0$, and the other variables are controlled as follows: there exist some constants $\beta, \beta^{\prime}$, and $\beta^{\prime \prime}$ (that depend only on $\theta$ ) such that

$$
0 \leq\left|y_{1}\right| \leq \beta x_{1}, \quad 0 \leq l_{1} \leq \beta^{\prime} x_{1}, \quad 0 \leq \xi_{1} \leq \beta^{\prime \prime} x_{1} .
$$


Therefore, the variables $\left|x_{1}-\mathrm{E}\left(x_{1}\right)\right|,\left|y_{1}-\mathrm{E}\left(y_{1}\right)\right|$ and $\left|l_{1}-\mathrm{E}\left(l_{1}\right)\right|,\left|\xi_{1}-\mathrm{E}\left(\xi_{1}\right)\right|$ also have exponential moments, which permits the control of the fluctuations of $X_{1}(k), Y_{1}(k), L_{1}(k)$, and $\Xi_{1}(k)$ around their means by Theorem 15 of [17, p. 52] and Lemma 5 of [17, p. 54] that we adapt slightly; if $Z_{k}=z_{1}+\cdots+z_{k}$, and the $z_{i}$ are centred and have exponential moments $\left(\mathrm{E}\left(\mathrm{e}^{t\left|z_{1}\right|}\right)<+\infty\right.$ for some $\left.t>0\right)$, then there exist constants $\alpha>0$ and $c>0$ such that

$$
\begin{gathered}
\mathrm{P}\left(\left|Z_{m}\right| \geq x \sqrt{m}\right) \leq 2 \exp \left(-c x^{2}\right) \quad \text { if } x \leq \alpha \sqrt{m}, \\
\mathrm{P}\left(\left|Z_{m}\right| \geq x \sqrt{m}\right) \leq 2 \exp (-c x \sqrt{m}) \quad \text { if } x \geq \alpha \sqrt{m},
\end{gathered}
$$

where the constants $c$ and $\alpha$ depend only on the distribution of $z_{1}$, but not on $m$ (the same constant $c$ can be chosen for both bounds; we do this). A consequence is the following proposition.

Proposition 2. Let $\left(x_{n}\right)$ be a sequence of real numbers such that $x_{n} \rightarrow+\infty$ and $x_{n}=O(\sqrt{n})$. There exists a constant $\gamma>0$ such that, for any large enough $m$,

$$
\mathrm{P}\left(\sup _{k \in \llbracket 1, m \rrbracket}\left|\frac{X_{1}^{\mathrm{DT}}(k)-k \mathrm{E}\left(x_{1}^{\mathrm{DT}}\right)}{\sqrt{m}}\right| \geq x_{m}\right) \leq 2 m \exp \left(-\gamma x_{m}\right) .
$$

The same results hold for $Y_{1}^{\mathrm{DT}}(k)-k \mathrm{E}\left(y_{1}^{\mathrm{DT}}\right), L_{1}^{\mathrm{DT}}(k)-k \mathrm{E}\left(l_{1}^{\mathrm{DT}}\right)$, and $\Xi_{1}^{\mathrm{DT}}(k)-k \mathrm{E}\left(\xi_{1}^{\mathrm{DT}}\right)$, with same $\gamma$ chosen for all cases.

Proof. The proofs for $X_{1}(k), Y_{1}(k), L_{1}(k)$, and $\Xi_{1}(k)$ are identical. For $X_{1}(k)$, write

$$
\mathrm{P}\left(\sup _{k \in \llbracket 1, m \rrbracket}\left|\frac{X_{1}(k)-k \mathrm{E}\left(x_{1}\right)}{\sqrt{m}}\right| \geq x_{m}\right) \leq \sum_{k=1}^{m} \mathrm{P}\left(\left|\frac{X_{1}(k)-k \mathrm{E}\left(x_{1}\right)}{\sqrt{k}}\right| \geq \frac{m^{1 / 2}}{\sqrt{k}} x_{m}\right),
$$

then use the first or second bound of (6) according to whether $m^{1 / 2} x_{m} / \sqrt{k} \leq \alpha \sqrt{k}$ or not.

Now, let $\left(x_{n}\right)$ be a sequence of real numbers such that $x_{n} \rightarrow+\infty$ and $x_{n}=O(\sqrt{n})$. There exists $\gamma>0$ such that, for any large enough $m$,

$$
\mathrm{P}\left(\sup _{k \in \llbracket 1, m \rrbracket}\left|\frac{X_{1}^{\mathrm{DT}}(k)+i Y_{1}^{\mathrm{DT}}(k)-k \mathrm{E}\left(x_{1}^{\mathrm{DT}}\right)}{\sqrt{m}}\right| \geq x_{m}\right) \leq 4 m \exp \left(-\gamma x_{m}\right) .
$$

To see this, use Proposition 2 and the fact that, for $u$ and $v$ in $\mathbb{R}$, if $|u+\mathrm{i} v| \geq a$ then $|u| \geq a / \sqrt{2}$ or $|v| \geq a / \sqrt{2}$.

In the case when the intensity of the PPP is constant and equal to $c>0$, for any $k \geq 1$,

$$
\begin{array}{ll}
X_{c}(k) \stackrel{\mathrm{D}}{=} \frac{1}{\sqrt{c}} X_{1}(k), & Y_{c}(k) \stackrel{\mathrm{D}}{=} \frac{1}{\sqrt{c}} Y_{1}(k), \\
L_{c}(k) \stackrel{\mathrm{D}}{=} \frac{1}{\sqrt{c}} L_{1}(k), & \Xi_{c}(k) \stackrel{\mathrm{D}}{=} \frac{1}{\sqrt{c}} \Xi_{1}(k) .
\end{array}
$$

This allows us to transfer results obtained under $\mathrm{P}_{1}$ to $\mathrm{P}_{c}$, since, for any Borelian $A$ in $\mathbb{R}^{k}$,

$$
\mathrm{P}\left(\left(X_{1}(1), \ldots, X_{1}(k)\right) \in A\right)=\mathrm{P}\left(\left(X_{c}(1), \ldots, X_{c}(k)\right) \in \sqrt{c} A\right) .
$$


3.3.3. Computations for directed $p_{\theta}$-Yao navigation. We use the same notation as in the previous part; this time, for $r>0$,

$$
\mathrm{P}\left(l_{1}^{\mathrm{DY}}>r\right)=\mathrm{P}_{1}(\# \mathrm{Cam}[k, 0](r) \cap \boldsymbol{S}=0)=\exp \left(-\frac{1}{2} r^{2} \theta\right),
$$

and $x_{1}^{\mathrm{DY}}=\cos (\alpha) l_{1}^{\mathrm{DY}}$ and $y_{1}^{\mathrm{DY}}=\sin (\alpha) l_{1}^{\mathrm{DY}}$ for a variable $\alpha$ uniform on $[-\theta / 2, \theta / 2]$, independent of $l_{1}^{\mathrm{DY}}$. Again, $l_{1}^{\mathrm{DY}}, x_{1}^{\mathrm{DY}}$, and $y_{1}^{\mathrm{DY}}$ have exponential moments, and we obtain $\mathrm{E}\left(y_{1}^{\mathrm{DY}}\right)=0$ and

$$
\mathrm{E}\left(l_{1}^{\mathrm{DY}}\right)=\sqrt{\frac{\pi}{2 \theta}}, \quad \mathrm{E}\left(x_{1}^{\mathrm{DY}}\right)=\mathrm{E}\left(l_{1}^{\mathrm{DY}}\right) \frac{\sin (\theta / 2)}{\theta / 2}=\boldsymbol{C}_{\mathrm{bis}}^{\mathrm{SY}}=\boldsymbol{C}_{\mathrm{bis}}^{\mathrm{CY}} .
$$

Moreover, since $\xi_{1}^{\mathrm{DY}}=l_{1}^{\mathrm{DY}} \cos (\theta / 2+V)$, where $V$ is uniform on $[-\theta / 2, \theta / 2]$ and independent of $l_{1}^{\mathrm{DY}}$,

$$
\mathrm{E}\left(\xi_{1}^{\mathrm{DY}}\right)=\mathrm{E}\left(l_{1}^{\mathrm{DY}}\right) \frac{\int_{-\theta / 2}^{\theta / 2} \cos (\alpha+\theta / 2) \mathrm{d} \alpha}{\theta}=\mathrm{E}\left(l_{1}^{\mathrm{DY}}\right) \frac{\sin (\theta)}{\theta}=C_{\mathrm{bor}}^{\mathrm{CY}},
$$

and, again, note that $\boldsymbol{Q}_{\mathrm{bor}}^{\mathrm{CY}}=\mathrm{E}\left(l_{1}^{\mathrm{DY}}\right) / \mathrm{E}\left(\xi_{1}^{\mathrm{DY}}\right)$ and $\boldsymbol{Q}_{\mathrm{bis}}^{\mathrm{CY}}=\mathrm{E}\left(l_{1}^{\mathrm{DY}}\right) / \mathrm{E}\left(x_{1}^{\mathrm{DY}}\right)$. This leads to the following result.

Proposition 3. In the DY case we have

$$
\left(\frac{L_{1}^{\mathrm{DY}}(k)}{k}, \frac{X_{1}^{\mathrm{DY}}(k)}{k}, \frac{Y_{1}^{\mathrm{DY}}(k)}{k}, \frac{\Xi_{1}^{\mathrm{DY}}(k)}{k}\right) \underset{k}{\stackrel{\text { a.s. }}{\longrightarrow}}\left(\mathrm{E}\left(l_{1}^{\mathrm{DY}}\right), \boldsymbol{C}_{\mathrm{bis}}^{\mathrm{CY}}, 0, \boldsymbol{C}_{\mathrm{bor}}^{\mathrm{CY}}\right) .
$$

Then

$$
\frac{L_{1}^{\mathrm{DY}}(k)}{X_{1}^{\mathrm{DY}}(k)} \underset{k}{\stackrel{\text { a.s. }}{\longrightarrow}} Q_{\text {bis }}^{\mathrm{CY}} \text { and } \frac{L_{1}^{\mathrm{DY}}(k)}{\Xi_{1}^{\mathrm{DY}}(k)} \underset{k}{\stackrel{\text { a.s. }}{\longrightarrow}} Q_{\text {bor }}^{\mathrm{CY}} \text {. }
$$

3.3.4. Computations for the random north model. Here we consider the case where the $n(s)$ are i.i.d. uniform on $[0,2 \pi]$. Let $\Delta^{\mathrm{RNT}}$ be the traveller stage (when going from $s$ to $t$ ), let $x^{\mathrm{RNT}}$ be the orthogonal projection of $\Delta^{\mathrm{RNT}}$ on the line $(s, t)$, and let $y^{\mathrm{RNT}}$ be the orthogonal projection on the orthogonal of $(s, t)$. A simple computation gives $\mathrm{E}\left(y_{1}^{\mathrm{RNT}}\right)=\mathrm{E}\left(y_{1}^{\mathrm{RNY}}\right)=0$,

$$
\begin{aligned}
& \mathrm{E}\left(x_{1}^{\mathrm{RNT}}\right)=\mathrm{E}\left(x_{1}^{\mathrm{DT}}\right) \int_{0}^{\theta / 2} \frac{\cos (v)}{\theta / 2} \mathrm{~d} v=\mathrm{E}\left(x_{1}^{\mathrm{DT}}\right) \frac{\sin (\theta / 2)}{\theta / 2}, \\
& \mathrm{E}\left(x_{1}^{\mathrm{RNY}}\right)=\mathrm{E}\left(l_{1}^{\mathrm{DY}}\right) \int_{0}^{\theta} \int_{0}^{\theta} \frac{\cos (v-\gamma)}{\theta^{2}} \mathrm{~d} \nu \mathrm{d} \gamma=\mathrm{E}\left(l_{1}^{\mathrm{DY}}\right) \frac{2-2 \cos (\theta)}{\theta^{2}} .
\end{aligned}
$$

The limiting quotients $\boldsymbol{Q}^{\mathrm{RNT}}$ and $\boldsymbol{Q}^{\mathrm{RNY}}$ are

$$
\boldsymbol{Q}^{\mathrm{RNT}}=\frac{\mathrm{E}\left(l_{1}^{\mathrm{DT}}\right)}{\mathrm{E}\left(x_{1}^{\mathrm{RNT}}\right)} \quad \text { and } \quad \boldsymbol{Q}^{\mathrm{RNY}}=\frac{\mathrm{E}\left(l_{1}^{\mathrm{DY}}\right)}{\mathrm{E}\left(x_{1}^{\mathrm{RNY}}\right)} .
$$

\subsection{From local to global or why differential equations come into play}

In Proposition 2 and in (7) a control of the difference between $X_{c}^{\mathrm{DT}}(k)$ and $Y_{c}^{\mathrm{DT}}(k)$ and their mean $k \mathrm{E}\left(x_{1}^{\mathrm{DT}}\right) / \sqrt{c}$ and 0 are given. If the intensity is $n f$, the 'local intensity' around $s$ is $n f(s)$. Roughly, the first stages of the traveller starting from $s$ are close in distribution under $\mathrm{P}_{n f}$ and under $\mathrm{P}_{n f(s)}$. Hence, at first order, $X_{n f}^{\mathrm{DT}}(k)$ should be close to $k \mathrm{E}\left(x_{1}^{\mathrm{DT}}\right) / \sqrt{n f(s)}$ : the speed of the traveller depends on position, and, therefore, a differential equation appears.

To approximate $\operatorname{Pos}^{\mathrm{DT}}$ by the solution of a differential equation, we split the traveller's trajectory into windows corresponding to some sequence of consecutive stages; to be relevant, the windows have to be small enough to keep the approximation of the local intensity $n f$ 
at $n f(s)$ and large enough to let the approximation $X_{n f}^{\mathrm{DT}}$ be $k \mathrm{E}\left(x_{1}^{\mathrm{DT}}\right) / \sqrt{n f(s)}$, that is, large enough to let large number-type compensations occur.

In this section we present the tools necessary to approximate the traveller's position by differential equations.

We start with some deterministic considerations. Let $D$ be an open subset of $\mathbb{R}^{d}$, and let $G: D \rightarrow \mathbb{R}^{d}$ be a Lipschitz function. Denote by $\operatorname{Eq}(G, z)$ the following ordinary differential equation

$$
\operatorname{Eq}(G, z):=\left\{\begin{array}{l}
y(0)=z \in D \\
y^{\prime}(x)=G(y(x))
\end{array} \quad \text { for } x \geq 0 .\right.
$$

This class of equation contains the family of ODEs defined in (2). By the Cauchy-Lipschitz theorem, $\operatorname{Eq}(G, z)$ admits a unique solution $y_{\mathrm{sol}(G, z)}$, or more simply $y_{\mathrm{sol}}$ when no confusion on $G$ and $z$ is possible. This solution is defined on a maximal interval $[0, \lambda(G, z))$, where $\lambda(G, z)$ is the hitting time of the border of $D$ by $y_{\text {sol }}$.

Before giving a convergence criterion for random trajectories, we present a deterministic criterion very close to the so-called explicit Euler scheme convergence theorem.

Lemma 1. Let $G: D \rightarrow \mathbb{R}^{d}$ be a Lipschitz function, $z \in D$ be fixed, and $\lambda \in[0, \lambda(G, z))$ be given.

Let $\left(a_{n}\right)$ and $\left(c_{n}\right)$ be two sequences of positive real numbers going to 0 , and let $\left(y_{n}\right)$ be a sequence of continuous functions from $\left[0, \lambda+a_{n}\right]$ to $D$ satisfying the following conditions.

(a) $y_{n}(0)=$ for all $n \geq 1$.

(b) For all $n \geq 1, y_{n}$ is linear between the points ( $\left.j a_{n}, j \in \llbracket 0,\left\lfloor\lambda / a_{n}\right\rfloor \rrbracket\right)$, and the slope of $y_{n}$ on these windows of size $a_{n}$ is well approximated by $G \circ y_{n}$ in the following sense:

$$
\sup _{j=0, \ldots,\left\lfloor\lambda / a_{n}\right\rfloor}\left|\frac{y_{n}\left((j+1) a_{n}\right)-y_{n}\left(j a_{n}\right)}{a_{n}}-G\left(y_{n}\left(j a_{n}\right)\right)\right| \leq c_{n} .
$$

Here we denote with an absolute value a norm in $\mathbb{R}^{d}$. Under this hypothesis, there exists $C_{\lambda}>0$ such that, for large enough $n$,

$$
\sup _{x \in[0, \lambda]}\left|y_{n}(x)-y_{\operatorname{sol}(G, z)}(x)\right| \leq C_{\lambda} \max \left\{a_{n}, c_{n}\right\} .
$$

Moreover, the constants $C_{\lambda}$ can be chosen in such a way that the function $\lambda \mapsto C_{\lambda}$ is bounded on all compact subsets of $[0, \lambda(G, z))$ and does not depend on the initial condition $z \in D$.

Proof. See Appendix A.

Note that we need $y_{n}$ to be defined on a slightly larger interval than $[0, \lambda]$ because of border effects.

We now extend this lemma to the convergence of a sequence of stochastic processes $\left(\boldsymbol{Z}_{n}\right)$.

Corollary 1. Let $G: D \rightarrow \mathbb{R}^{n}$ be a Lipschitz function, $z \in D$ be fixed, and $\lambda \in[0, \lambda(G, z))$ be given.

Let $\left(a_{n}\right),\left(b_{n}\right),\left(c_{n}\right),\left(c_{n}^{\prime}\right)$, and $\left(d_{n}\right)$ be five sequences of positive real numbers going to 0 ; let $\left(\boldsymbol{Z}_{n}\right)$ be a sequence of continuous stochastic processes from $\left[0, \lambda+a_{n}\right]$ to $D$ satisfying the following conditions.

(a) $Z_{n}(0)=z$ a.s. 
(b) The slope of $\boldsymbol{Z}_{n}$ on windows of size $a_{n}$ is well approximated by $G \circ \boldsymbol{Z}_{n}$, with a large probability:

$$
\sup _{j \in \llbracket 0, \lambda / a_{n} \rrbracket} \mathrm{P}\left(\left|\frac{\boldsymbol{Z}_{n}\left((j+1) a_{n}\right)-\boldsymbol{Z}_{n}\left(j a_{n}\right)}{a_{n}}-G\left(\boldsymbol{Z}_{n}\left(j a_{n}\right)\right)\right| \geq c_{n}\right) \leq a_{n} d_{n} .
$$

(c) Inside the windows, the fluctuations of $\boldsymbol{Z}_{n}$ are small:

$$
\sup _{j \in \llbracket 0, \lambda / a_{n} \rrbracket} \mathrm{P}\left(\sup _{x \in\left[j a_{n},(j+1) a_{n}\right]}\left|\boldsymbol{Z}_{n}(x)-\boldsymbol{Z}_{n}\left(j a_{n}\right)-\left(x-j a_{n}\right) G\left(\boldsymbol{Z}_{n}\left(j a_{n}\right)\right)\right| \geq c_{n}^{\prime}\right) \leq a_{n} b_{n} .
$$

If these three conditions are satisfied then

$$
\mathrm{P}\left(\sup _{x \in[0, \lambda]}\left|y_{\operatorname{sol}(G, z)}(x)-Z_{n}(x)\right| \leq C_{\lambda} \max \left\{a_{n}, c_{n}, c_{n}^{\prime}\right\}\right) \geq 1-(\lambda+1)\left(d_{n}+b_{n}\right)
$$

for a function $\lambda \mapsto C_{\lambda}$ independent of $z$, bounded on every compact subset of $[0, \lambda(G, z))$.

Proof. See Appendix A.

Note that Corollary 1(c) contains (b) if $c_{n}^{\prime} \leq c_{n} a_{n}$, which will be the case in the applications we have, but the present presentation allows us to better understand the underlying phenomenon.

\subsection{On the largest stage and the maximum number of points in a ball}

The quantity

$$
\operatorname{Navmax}[\theta](S):=\sup _{s \in \mathscr{D}[a], \eta \in[0,2 \pi]} \inf \left\{h: \#\left(S \cap\left(s+\mathrm{e}^{\mathrm{i} \eta} \operatorname{Cam}(\theta)(h)\right)\right) \geq 1\right\}
$$

is a bound on the largest stages length for all starting points $(s \in \mathscr{D}[a])$ and targets $(t \in \mathcal{D})$ and all navigations considered in this paper.

Lemma 2. For any $\theta \in(0,2 \pi]$ and any $C>0$, if $n$ is large enough,

$$
\mathrm{P}_{n f}\left(\mathrm{Navmax}[\theta] \geq n^{C-1 / 2}\right) \leq \exp \left(-n^{C / 2}\right) .
$$

Proof. Consider a tiling of the plane with squares of size $n^{-1 / 2+C / 2}$. For large enough $n$, each element of the family $\left(s+\mathrm{e}^{\mathrm{i} \eta} \operatorname{Cam}(\theta)\left(n^{-1 / 2+C}\right), s \in \mathbb{C}, \eta \in[0,2 \pi]\right)$ contains in its interior at least a square of the tiling. It suffices then to show that any square $(\square)$ of the tiling intersecting $\mathscr{D}[a]$ intersects $S$ also. But, $\mathrm{P}_{n f}(\# S \cap \square=0)=\exp \left(-\int_{\square} n f(z) \mathrm{d} z\right) \leq \exp \left(-m_{f} n^{C}\right)$ since $n f \geq n m_{f}$, and the area of $\square$ is $n^{-1+C}$. Since $O\left(n^{1-C}\right)$ squares intersect the bounded domain $\mathscr{D}[a]$, by the union bound, the probability that there exists a square containing no elements of $S$ is $O\left(n^{1-C} \exp \left(-m_{f} n^{C}\right)\right)$.

Now we turn our attention to

$$
\operatorname{Maxball}[r](S):=\max \{\#(S \cap B(x, r)) \mid x \in \mathscr{D}[a]\},
$$

the maximum number of elements of $S$ in a ball with radius $r$ and centre in $\mathscr{D}[a]$.

Lemma 3. For any $B>0$ and $\varepsilon>0$, if $n$ is large enough,

$$
\mathrm{P}_{n f}\left(\operatorname{Maxball}\left[n^{-1 / 2+B}\right] \geq n^{2 B+\varepsilon}\right) \leq \exp \left(-n^{2 B+\varepsilon}\right) .
$$


Note that, for $s \in \mathcal{D}$, the mean number of elements in $B\left(s, n^{-1 / 2+B}\right) \cap \boldsymbol{S}(n f)$ is $O\left(n^{2 B}\right)$.

Proof of Lemma 3. Consider a tiling of the plane with squares of size $a_{n}=n^{B-1 / 2}$; denote by $\mathrm{Squ}_{a}$ the subset of squares whose distance to $\mathscr{D}[a]$ is smaller than $a_{n}$. Any disk $B\left(x, a_{n}\right)$ with $x \in \mathscr{D}[a]$ intersects a bounded number $d$ of such squares, with $d$ independent of $B$. Hence, for any positive sequence $\left(b_{n}\right)$,

$$
\left\{\operatorname{Maxball}\left[a_{n}\right](S(n f)) \geq b_{n}\right\} \subset\left\{\sup _{\square \in \mathrm{Squ}_{a}} \#(S(n f) \cap \square) \geq \frac{b_{n}}{d}\right\} .
$$

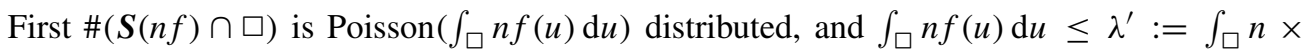
$M_{f} \mathrm{~d} u=n a_{n}^{2} M_{f}$. Secondly, for $X \sim \operatorname{Poisson}(\lambda)$ and $\lambda^{\prime}>\lambda, \mathrm{P}(X \geq r)=\sup _{t>0} \mathrm{P}\left(\mathrm{e}^{t X} \geq\right.$ $\left.\mathrm{e}^{t r}\right) \leq \sup _{t>0} \mathrm{e}^{-t r+\lambda^{\prime}\left(\mathrm{e}^{t}-1\right)}$. By a simple (classical) optimisation on $t$, we obtain

$$
\mathrm{P}_{n f}\left(\#(S \cap \square)>\frac{b_{n}}{d}\right) \leq \exp \left(-\frac{b_{n}}{d} \ln \left(\frac{b_{n}}{d n a_{n}^{2} M_{f}}\right)+\frac{b_{n}}{d}-n a_{n}^{2} M_{f}\right) .
$$

To end, take $a_{n}=n^{-1 / 2+B}$ and $b_{n}=n^{2 B+\varepsilon}$, and apply the union bound to the $O\left(1 / a_{n}^{2}\right)$ squares of $\mathrm{Squ}_{a}$.

For $C>0, B>0$, and $\varepsilon>0$, consider the following events:

$$
\Omega(n, C):=\left\{\operatorname{Navmax}[\theta] \leq n^{C-1 / 2}\right\}, \quad \Omega_{n, \varepsilon}:=\left\{\operatorname{Maxball}\left[n^{B-1 / 2}\right] \leq n^{(2+\varepsilon) B}\right\} \cap \Omega(n, C) .
$$

\subsection{About the constants in this paper}

The aim of this section is to discuss the role of the constants in this paper, and maybe to help the reader follow the computations more easily.

- We will use $n^{C-1 / 2}$ as a bound on Navmax; this bound is valid with probability 1 $\exp \left(-n^{C / 2}\right)$ for large $n$.

- The behaviour of the traveller close to its target is treated in Section 3.2. 'Far from its target' (or to some particular point in the trajectory) means $|t-s| \geq n^{B-1 / 2}$. When the traveller enters the final ball $B\left(t, n^{B-1 / 2}\right)$, at most Maxball $\left[n^{B-1 / 2}\right]$ additional stages will be necessary to reach $t$, that is, at most $n^{3 B}$ stages with probability $1-\exp \left(-n^{3 B}\right)$.

- In Corollary 1, when the approximation by the solution of a differential equation is needed, a window with size $a_{n}$ arises. We will take

$$
a_{n}=n^{w-1 / 2} \text {. }
$$

Taking into account the space normalisation, this corresponds to considering $n^{w}$ stages of the traveller.

Some assumptions are made on the relative values of $w, C$, and $B$ (for example, $2 w+3 C<$ $\left.\frac{1}{2}\right)$ in the statements of this paper. In any case, there is a choice of $(w, C, B)$ which fulfills all the requirements of the intermediate results, that is,

$$
0<w<\frac{1}{4}, \quad 2 w+3 C<\frac{1}{2}, \quad C+w<B, \quad 0<B<\frac{1}{4}, \quad C \in\left(0, \frac{1}{2}\right),
$$

as can be easily checked. Another quantity appearing later is $w^{\prime} \in\left(0, \frac{1}{2}\right)$ related to the bound $n^{\alpha}$, where $\alpha<\frac{1}{8}$, which appears in most of the important theorems. This $\alpha$ appears to be $\max \left\{w\left(w^{\prime}-\frac{1}{2}\right), w\left(w^{\prime}+\frac{1}{2}\right)-\frac{1}{2}, w-\frac{1}{2}\right\}$. To reach an $\alpha$ close to $\left(\frac{1}{8}\right)^{-}$, take $w=\left(\frac{1}{4}\right)^{-}, C=$ $0^{+}, B=\left(\frac{1}{4}\right)^{-}$, and $w^{\prime}=0^{+}$. 


\subsection{Navigations in homogeneous and nonhomogeneous PPPs}

Here we define the notions of simple stages and nonintersecting decision domains.

Definition 7. Let $\left(d_{1}, \ldots, d_{k}\right)$ be the $k$ first stages of a traveller going from $s$ to $t$. We say that these stages are $\boldsymbol{X}(s, t)$-simple if the corresponding decision domains do not intersect; this amounts to saying that if the traveller uses as a set of possible stops $\left\{s+\sum_{j=1}^{i} d_{i}, i \leq k\right\}$ then the decision sectors are nonintersecting when going from $s$ to $t$ (see Figure 8). A Borelian subset $\Theta$ of $\mathbb{C}^{k}$ is said to be $\boldsymbol{X}(s, t)$-simple if, for any sequence $\left(d_{1}, \ldots, d_{k}\right) \in \Theta$, the stages $\left(d_{1}, \ldots, d_{k}\right)$ are $\boldsymbol{X}(s, t)$-simple.

Proposition 4 below is really important. It provides, around a point $s$, a bound for the deviations of the traveller stages under the nonhomogeneous $\mathrm{P}_{n f}$ using the much simpler measure $\mathrm{P}_{n f(s)}$. We were unable to find a sufficient coupling argument and so instead we use an argument based on the comparison of distributions.

Let us set the following vectorial notation: $\Delta^{\mathrm{DT}}[s, j]:=\left(\Delta^{\mathrm{DT}}(s, 1), \ldots, \Delta^{\mathrm{DT}}(s, j)\right)$; in the sequel, $\Delta^{X}[s, t, j]$ and similar notation will be used for the navigation processes $\boldsymbol{X}$ if we need to specify the target.

Proposition 4. Let $\boldsymbol{X} \in\{\mathrm{CY}, \mathrm{CT}\}$ and $\theta \leq \pi / 3$, or $\boldsymbol{X}=\mathrm{SY}$ and $\theta<\pi / 2$, or $\boldsymbol{X}=\mathrm{ST}$ and $\theta \leq \pi / 2$, or $\boldsymbol{X}=\mathrm{DT}$ and $\theta \leq \pi$, or $\boldsymbol{X}=\mathrm{DY}$ and $\theta \leq \pi / 2$. Assume that $\left(\gamma_{n}\right)$ is a sequence of integers such that $\gamma_{n} \sim n^{w}$ for some $w \in\left(0, \frac{1}{4}\right)$. Let $C \in\left(0, \frac{1}{2}\right)$ such that $2 w+3 C<\frac{1}{2}$, and let $(s, t) \in \mathscr{D}^{\prime}[a]$. For any $\Theta$ Borelian subset of $\mathbb{C}^{\gamma_{n}} \boldsymbol{X}(s, t)$-simple and large enough $n$,

$$
\mathrm{P}_{n f}\left(\Delta^{X}\left[s, t, \gamma_{n}\right] \in \Theta\right) \leq \exp \left(-n^{C / 2}\right)+2 \mathrm{P}_{n f(s)}\left(\Delta^{X}\left[s, t, \gamma_{n}\right] \in \Theta\right),
$$

where the argument $t$ is omitted if $\boldsymbol{X} \in\{\mathrm{DT}, \mathrm{DY}\}$, since the target does not exist in these cases.

Proposition 4 establishes a very local property since after $n^{w}$ steps the traveller is, with large probability, at a distance $n^{w+C-1 / 2} \ll 1$ from its starting point, and still in $\mathscr{D}$ for large $n$.

It follows from Proposition 4 that an event with small probability under $\mathrm{P}_{n f(s)}$, for example the events considered in Proposition 2, is also small under $\mathrm{P}_{n f}$.

Remark 3. The simplicity of the stages is unlikely to arise when $s$ is close to $t$ since the decision domains lose the property of 'a constant' direction. For this reason, Proposition 4 will be used only when $s$ is far from $t$. Note also that the factor 2 on the right-hand side of (14) is taken for convenience, any number greater than 1 would suffice.

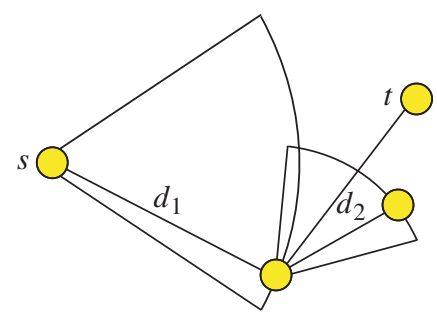

FIGURE 8: An example of the configuration of points in the straight Yao navigation leading to an intersection of the decision domains. For DT navigation, the decision sectors are simple for $\theta \leq \pi$, and, for DY navigation, the condition is $\theta \leq \pi / 2$. For ST and SY navigation, for any $\theta$, the decision sectors can intersect. 
Proof of Proposition 4. By Lemma 2, for large $n, \mathrm{P}_{n f}(\complement \Omega(n, C)) \leq \exp \left(-n^{C / 2}\right)$. Write

$$
\mathrm{P}_{n f}\left(\Delta^{X}\left[s, t, \gamma_{n}\right] \in \Theta\right) \leq \mathrm{P}_{n f}(\complement \Omega(n, C))+\mathrm{P}_{n f}\left(\Delta^{X}\left[s, t, \gamma_{n}\right] \in \Theta, \Omega(n, C)\right) .
$$

We then just have to bound the second term on the right-hand side. For this, we will show that

$$
\begin{aligned}
\mathrm{P}_{n f}\left(\Delta^{X}\left[s, t, \gamma_{n}\right] \in \Theta, \Omega(n, C)\right) & \leq 2 \mathrm{P}_{n f(s)}\left(\Delta^{X}\left[s, t, \gamma_{n}\right] \in \Theta, \Omega(n, C)\right) \\
& \leq 2 \mathrm{P}_{n f(s)}\left(\Delta^{X}\left[s, t, \gamma_{n}\right] \in \Theta\right),
\end{aligned}
$$

proving only the first inequality. Furthermore, we will show that it is easy to see, under $\mathrm{P}_{n f}$ and under $\mathrm{P}_{n f(s)}$, that the distribution of $\Delta^{X}\left[s, \gamma_{n}\right]$ has a density with respect to the Lebesgue measure on $\mathbb{C}^{\gamma_{n}}$, denoted by $\boldsymbol{D}_{1}$ and $\boldsymbol{D}_{2}$, respectively. (More exactly, the representation of the probability measure of $\Delta^{X}\left[s, \gamma_{n}\right]$ using densities holds when small stages are considered, small enough such that all decision sectors considered are included in $\mathscr{D}$ and the target is not reached after the first $\gamma_{n}$ steps.) We will then compare these densities and bound their ratio on a set of interest.

Since the distribution of the stages depends on decision sectors that have a shape depending on $\boldsymbol{X}$, it is useful here to introduce some notation. Denote by $d^{X}(s, t)$ the decision domain (a Camembert section or a triangular domain) corresponding to $\Delta(s, t)^{\boldsymbol{X}}$, and by $\partial d^{\boldsymbol{X}}(s, t)$ the corresponding principal boundaries, namely, depending on $X$, the side of the triangle which is not on the decision sector or the arc of a circle in the Camembert section. We write with an absolute value the Lebesgue measures of these sets. Finally, let $\operatorname{Seg}^{X}(s, t)$ be the length of the segment of the plane starting from $s$, supported by the bisecting line of the decision sector, and whose second end is on $\partial d^{X}(s, t)$.

Under $\mathrm{P}_{n f}$ or $\mathrm{P}_{n f(s)}$, conditionally on $\partial d^{X}(s, t)$, the density of the law of $\Delta^{X}(s, t)$ is respectively proportional to $n f(s+\cdot)$ or uniform on $\partial d^{X}(s, t)$. To characterise the law of $\Delta^{X}(s, t)$, it remains to express the distribution of $\left|\operatorname{Seg}^{X}(s, t)\right|$ :

$$
\mathrm{P}_{n f}\left(\left|\operatorname{Seg}^{X}(s, t)\right|>x\right)=\exp \left(-\int_{d^{X}(s, t, x)} n f(u) \mathrm{d} u\right) .
$$

Here $d^{X}(s, t, x)$ is the decision domain such that $\left|\operatorname{Seg}^{X}(s, t)\right|=x$. The density of $\left|\operatorname{Seg}^{X}(s, t)\right|$ is then

$$
\left(\int_{\partial d^{X}(s, t, x)} n f(u) \mathrm{d} u\right) \exp \left(-\int_{d^{X}(s, t, x)} n f(u) \mathrm{d} u\right) .
$$

The density of the same variable under $\mathrm{P}_{n f(s)}$ is obtained by replacing $f(u)$ by the constant value $f(s)$ in this formula. Finally, under $\mathrm{P}_{n f}$, the density of $\Delta^{\boldsymbol{X}}(s, t)$ is

$$
g_{n f, s, t}(\delta)=f(s+\delta) \exp \left(-\int_{d^{X}(s, t, x(\delta))} n f(u) \mathrm{d} u\right)
$$

for any $\delta$ in the decision sector of the traveller going from $s$ to $t$ using $\boldsymbol{X}$, where $x(\delta)$ is the length of $\left|\operatorname{Seg}^{X}(s, t)\right|$ corresponding to stage $\delta$ (again, this holds for 'small stages'); under $\mathrm{P}_{n f(s)}$,

$$
g_{n f(s), s, t}(\delta)=f(s) \exp \left(-\int_{d^{X}(s, t, x(\delta))} n f(s) \mathrm{d} u\right),
$$

where the value of this integral is $n f(s)\left|d^{X}(s, t, x)\right|=n f(s)\left|d^{X}(\cdot, \cdot, x)\right|$. Now, let us treat several stages $v:=\left(\delta_{1}, \ldots, \delta_{\gamma_{n}}\right) \in \Theta$. We will use two ingredients. First, the simplicity of $\Theta$, which guarantees the nonintersection of the decision domains; for this, we use (15) and (16), 
successively. Second, under the nonintersecting condition, by successively conditioning on the first stages of the traveller, it appears that $\boldsymbol{D}_{1}$ and $\boldsymbol{D}_{2}$ have the multiplicative forms

$$
\boldsymbol{D}_{1}(\mathrm{v})=\prod_{i=1}^{\gamma_{n}} g_{n f(s), s_{i-1}, t}\left(\delta_{i}\right), \quad \boldsymbol{D}_{2}(\mathrm{v})=\prod_{i=1}^{\gamma_{n}} g_{n f, s_{i-1}, t}\left(\delta_{i}\right),
$$

where $s_{0}=s$ and $s_{j}=s_{0}+\delta_{1}+\cdots+\delta_{j}$. The sequence $\left(d^{X}\left(s_{i}, t, x\left(\delta_{i}\right)\right), i=1, \ldots, \gamma_{n}\right)$ is the same in both formulae, and so $\boldsymbol{D}_{1}$ and $\boldsymbol{D}_{2}$ are very similar. Let us see why $\boldsymbol{D}_{2}(\mathrm{v}) \leq 2 \boldsymbol{D}_{1}(\mathrm{v})$ holds for any element $\mathrm{v}$ in $\Omega(n, C) \cap \Theta$, which will be enough to complete the proof.

For this, consider the first factor $\prod_{i=1}^{\gamma_{n}} f\left(s_{i-1}+\delta_{i}\right)$ appearing in $\boldsymbol{D}_{2}$. If $\mathbf{v} \in \Omega(n, C)$, for $m \leq \gamma_{n}$, since $f$ is Lipschitzian,

$$
\prod_{i=1}^{\gamma_{n}} f\left(s_{i-1}+\delta_{i}\right) \leq \prod_{i=1}^{\gamma_{n}} f(s)+\alpha_{f} \gamma_{n} n^{C-1 / 2} \leq f(s)^{\gamma_{n}}\left(1+c_{1} \gamma_{n} n^{C-1 / 2}\right)^{\gamma_{n}}
$$

for constants $c_{1}>0$ and $\alpha_{f}$, since $f$ is bounded. Let us bound the second factor appearing in $\boldsymbol{D}_{2}$ :

$$
\begin{aligned}
\exp \left(-n \int_{d^{X}{ }_{\left(s_{m-1}, t, \delta_{m}\right)}} f(u) \mathrm{d} u\right)= & \exp \left(-n \int_{d^{X}\left(s_{m-1}, t, \delta_{m}\right)} f(u)-f(s) \mathrm{d} u\right) \\
& \times \exp \left(-n \int_{d^{X_{\left(s_{m-1}, t, \delta_{m}\right)}}} f(s) \mathrm{d} u\right) .
\end{aligned}
$$

The second term in this product is simply $\exp \left(-n f(s)\left|d^{X}\left(s_{m-1}, t, \delta_{m}\right)\right|\right)$; let us bound the first term. Using the facts that the area of $d^{X}\left(s_{m-1}, t, \delta_{m}\right)$ is bounded by $c_{2} n^{2 C-1}$ (since $\delta_{m} \leq n^{C-1 / 2}$ ) and $f(u)-f(s)$ is greater than $-\alpha_{f} m n^{C-1 / 2}$ (Lipschitz, and since we are in $\left.\Omega(n, C),|t-s| \leq m n^{C-1 / 2}\right)$, the left-hand side of (17) is bounded by

$$
\exp \left(-n \int_{d^{X}\left(s_{m-1}, t, \delta_{m}\right)} f(u)-f(s) \mathrm{d} u\right) \leq \exp \left(c_{2} n m n^{C-1 / 2} n^{2 C-1}\right)=\exp \left(c_{2} m n^{3 C-1 / 2}\right)
$$

for some constant $c_{2}>0$. Finally, putting together the $\gamma_{n}$ terms involved we obtain

$$
\boldsymbol{D}_{2}(\mathrm{v}) \leq \boldsymbol{D}_{1}(v)\left(1+c_{1} \gamma_{n} n^{C-1 / 2}\right)^{\gamma_{n}} \prod_{m=1}^{\gamma_{n}} \exp \left(c_{2} m n^{3 C-1 / 2}\right) \text {. }
$$

Recall that $\gamma_{n} \sim n^{w} \rightarrow+\infty$. The second term, $\left(1+c_{1} \gamma_{n} n^{C-1 / 2}\right)^{\gamma_{n}}$, goes to 1 if $C<\frac{1}{2}$. The third term goes to 1 if $\sum_{m=1}^{\gamma_{n}} c_{2} m n^{3 C-1 / 2}$ goes to 0 , that is, if $2 w+3 C-\frac{1}{2}<0$. Finally, taking $C \in\left(0, \frac{1}{2}\right)$ and $w>0$ satisfying $2 w+3 C-\frac{1}{2}<0$, we see that $\left(1+c_{1} \gamma_{n} n^{C-1 / 2}\right)^{\gamma_{n}} \times$ $\prod_{m=1}^{\gamma_{n}} \exp \left(c_{2} m n^{3 C-1 / 2}\right) \rightarrow 1$, and, thus, is less than 2 for large enough $n$.

\subsection{Limiting behaviour for a traveller using DT or DY navigation}

In this section we deal with DT navigation, but DY navigation can be studied similarly. The aim is to show that, when $n \rightarrow+\infty$, after rescaling, the function $\operatorname{Pos}_{s}^{\mathrm{DT}}$ under $\mathrm{P}_{n f}$ converges in distribution to $\operatorname{Sol}_{s} \boldsymbol{C}_{\text {bis }}^{\mathrm{CT}}, 0$, the solution of $\operatorname{ODE}\left(\boldsymbol{C}_{\text {bis }}^{\mathrm{CT}}, 0, s\right)$ as defined in (2).

Theorem 5. Let $\boldsymbol{X}=\mathrm{DT}$ and $\theta \leq \pi$, or $\boldsymbol{X}=\mathrm{DY}$ and $\theta \leq \pi / 2$. For any $s \in \mathscr{D}[a]$, any $\alpha \in\left(0, \frac{1}{8}\right)$, and $\lambda \in\left[0, \lambda\left(F_{C_{\text {bis }}^{X}, 0}, s\right)\right)$, there exists a constant $d>0$ such that, for large enough $n$,

$$
\mathrm{P}_{n f}\left(\sup _{x \in[0, \lambda]}\left|\operatorname{Pos}_{s}^{\mathrm{DT}}(x \sqrt{n})-\operatorname{Sol}_{s}^{C_{\text {bis }}^{\mathrm{CT}}, 0}(x)\right| \leq n^{-\alpha}\right) \geq 1-\exp \left(-n^{d}\right) .
$$


Note that $\operatorname{ODE}\left(\boldsymbol{C}_{\text {bis }}^{\mathrm{CT}}, 0, s\right)$ coincides with $\operatorname{Eq}\left(F_{\boldsymbol{C}_{\text {bis }}^{\mathrm{CT}}, 0}, s\right)$, as introduced in (10) (see also the considerations about $\lambda(G, z)$, the hitting time of $\complement \mathscr{D}$ by the solution of $\operatorname{Eq}(G, z))$. Hence, Theorem 5 is a consequence of the following lemma.

Lemma 4. Let $\lambda \in\left[0, \lambda\left(F_{C_{\text {bi }}^{\mathrm{CT}}, 0}, s\right)\right), \gamma_{n}=\left\lfloor n^{w}\right\rfloor$, and $a_{n}=\gamma_{n} / \sqrt{n} \sim n^{w-1 / 2}$ be the size of the 'windows'. Let $C, w$, and $w^{\prime}$ be some positive constants such that $2 w+3 C-\frac{1}{2}<0$ and $w^{\prime}<\frac{1}{2}$. Then, for any $s \in \mathscr{D}[a]$, the sequence $\left(x \mapsto \operatorname{Pos}_{s}^{\mathrm{DT}}(x \sqrt{n})\right)$ satisfies the hypothesis of Corollary 1 for $G=F_{C_{\text {bis }}^{\mathrm{CT}}, 0}, c_{n}=n^{w\left(w^{\prime}-1 / 2\right)} / \sqrt{m_{f}}, b_{n}=\exp \left(-n^{\min \left(w w^{\prime} / 2, C / 3\right)}\right), c_{n}^{\prime}=$ $n^{w\left(w^{\prime}+1 / 2\right)-1 / 2} / \sqrt{m_{f}}$, and $d_{n}=b_{n}$. Thus,

$$
\mathrm{P}_{n f}\left(\sup _{x \in[0, \lambda]}\left|\operatorname{Sol}_{s}^{C_{\text {bis }}^{\mathrm{CT}}, 0}(x)-\operatorname{Pos}_{s}^{\mathrm{DT}}(x \sqrt{n})\right| \leq C_{\lambda} \max \left\{a_{n}, c_{n}^{\prime}, c_{n}\right\}\right) \geq 1-2(\lambda+1) b_{n}
$$

for $\lambda \rightarrow C_{\lambda}$ bounded on compact sets.

Here, the minimum value for $\max \left\{a_{n}, c_{n}, c_{n}^{\prime}\right\}$ is $n^{-1 / 8+}$, as explained in Section $3.6(w=$ $\left(\frac{1}{4}\right)^{-}, C=0^{+}$, and $w^{\prime}=0^{+}$).

In the homogeneous PPP $\mathrm{P}_{n c}$ (for some $c>0$ ), the correct order for the variance of $\operatorname{Pos}_{s}^{\mathrm{DT}}(x \sqrt{n})$ is $1 / \sqrt{n}$, since it is a sum of $\sqrt{n} \lambda$ random variables with variance of order $1 / n$ by (4). Then standard deviations have order $n^{1 / 4}$. Here the constant $\frac{1}{8}$ arising in the results is not so good, but gives the exponential bounds needed here, which are also valid for nonhomogeneous PPPs.

Proof of Lemma 4. For short, we write $\boldsymbol{Z}_{s}^{(n)}(x)$ instead of $\operatorname{Pos}_{s}^{\mathrm{DT}}(x \sqrt{n})$. We will use Proposition 2, and (7) and (9), and we will establish some bounds valid for the first $\gamma_{n}$ stages of a traveller starting from a generic point $s_{0} \in \mathbb{C}$ (that is, $\left.\boldsymbol{Z}_{s}^{(n)}(0)=s_{0}\right)$. Recall that $\boldsymbol{C}_{\text {bis }}^{\mathrm{CT}}=\mathrm{E}\left(x_{1}^{\mathrm{DT}}\right)$, and consider the following Borelian subset of $\mathbb{C}^{\gamma_{n}}$ :

$$
\Theta_{n}^{(1)}:=\left\{\left(\lambda_{1}, \ldots, \lambda_{\gamma_{n}}\right) \in \mathbb{C}^{\gamma_{n}}, \sup _{l \in \llbracket 1, \gamma_{n} \rrbracket}\left|\sum_{j=1}^{l}\left(\lambda_{j}-\frac{C_{\text {bis }}^{\mathrm{CT}}}{\sqrt{n f\left(s_{0}\right)}}\right)\right| \leq y_{n}\right\}
$$

for $\left(y_{n}\right)$, a sequence that will be fixed later; note that $\boldsymbol{C}_{\mathrm{bis}}^{\mathrm{CT}} / \sqrt{f\left(s_{0}\right)}=F_{\boldsymbol{C}_{\mathrm{bis}}^{\mathrm{CT}}, 0}\left(s_{0}\right)=F_{\boldsymbol{C}_{\mathrm{bis}}^{\mathrm{CT}}, 0}$ $\left(\boldsymbol{Z}_{s}^{(n)}(0)\right)$. In terms of events,

$$
\left\{\Delta^{\mathrm{DT}}\left[s_{0}, \gamma_{n}\right] \in \Theta_{n}^{(1)}\right\}=\left\{\sup _{l \in \llbracket 1, \gamma_{n} \rrbracket}\left|\sum_{j=1}^{l}\left(\Delta^{\mathrm{DT}}\left(s_{0}, j\right)-\frac{C_{\mathrm{bis}}^{\mathrm{CT}}}{\sqrt{n f\left(s_{0}\right)}}\right)\right| \leq y_{n}\right\} .
$$

Using $\boldsymbol{Z}_{s_{0}}^{(n)}(j / \sqrt{n})=s_{0}+\sum_{l=1}^{j} \Delta^{\mathrm{DT}}\left(s_{0}, l\right)$,

$$
\left\{\Delta^{\mathrm{DT}}\left[s_{0}, \gamma_{n}\right] \in \Theta_{n}^{(1)}\right\}=\left\{\sup _{l \in \llbracket 1, \gamma_{n} \rrbracket}\left|\boldsymbol{Z}_{s_{0}}^{(n)}\left(\frac{l}{\sqrt{n}}\right)-s_{0}-l \frac{\boldsymbol{C}_{\mathrm{bis}}^{\mathrm{CT}}}{\sqrt{n f\left(s_{0}\right)}}\right| \leq y_{n}\right\} .
$$

Therefore, the event $\Theta_{n}^{(1)}$ contains (for $l=\gamma_{n}$ )

$$
\left\{\left|\frac{\boldsymbol{Z}_{s_{0}}^{(n)}\left(a_{n}\right)-s_{0}}{a_{n}}-F_{C_{\text {bis }}^{\mathrm{CT}}, 0}\left(\boldsymbol{Z}_{s_{0}}^{(n)}(0)\right)\right| \leq \frac{y_{n}}{a_{n}}\right\}
$$

and

$$
\left\{\sup _{x \in\left[0, a_{n}\right]}\left|\boldsymbol{Z}_{s_{0}}^{(n)}(x)-\boldsymbol{Z}_{s_{0}}^{(n)}(0)-(x-0) F_{\boldsymbol{C}_{\text {bis }}^{\mathrm{CT}}, 0}\left(\boldsymbol{Z}_{s_{0}}^{(n)}(0)\right)\right| \leq y_{n}\right\} .
$$


We now take $y_{n}=x_{\gamma_{n}} \sqrt{\gamma_{n}} / \sqrt{n m_{f}}$ for $x_{n} \sim n^{w^{\prime}}$. Since $x_{n}=O(\sqrt{n})$, it fulfills the requirement of Proposition 2. Moreover, since $2 w+3 C-\frac{1}{2}<0$, the comparison provided by Proposition 4 is valid and, thus, we work for now under $\mathrm{P}_{n f\left(s_{0}\right)}$. We obtain, rescaling (8),

$$
\begin{aligned}
\mathrm{P}_{n f\left(s_{0}\right)} & \left(\Delta^{\mathrm{DT}}\left[s_{0}, \gamma_{n}\right] \in \mathrm{C} \Theta_{n}^{(1)}\right) \\
& =\mathrm{P}_{n f\left(s_{0}\right)}\left(\sup _{l \in \llbracket 1, \gamma_{n} \rrbracket}\left|\sum_{j=1}^{l}\left(\Delta^{\mathrm{DT}}\left(s_{0}, j\right)-\frac{\boldsymbol{C}_{\text {bis }}^{\mathrm{CT}}}{\sqrt{n f\left(s_{0}\right)}}\right)\right| \geq \frac{x_{\gamma_{n}} \sqrt{\gamma_{n}}}{\sqrt{n m_{f}}}\right) \\
& \leq \mathrm{P}_{1}\left(\sup _{l \in \llbracket 1, \gamma_{n} \rrbracket}\left|\sum_{j=1}^{l} \frac{\Delta^{\mathrm{DT}}\left(s_{0}, j\right)-C_{\text {bis }}^{\mathrm{CT}}}{\sqrt{\gamma_{n}}}\right| \geq x_{\gamma_{n}}\right) \\
& \leq 2 \gamma_{n} \exp \left(-\gamma^{\prime} x_{\gamma_{n}}\right) \\
& \leq \exp \left(-\gamma^{\prime \prime} n^{w w^{\prime}}\right)
\end{aligned}
$$

for constants $\gamma^{\prime}>0$ and $\gamma^{\prime \prime}>0$, and large enough $n$ (where we have used the fact that $\sqrt{f\left(s_{0}\right)} / \sqrt{m_{f}} \geq 1$ ). Take $c_{n}=x_{\gamma_{n}} / \sqrt{\gamma_{n} m_{f}} \sim n^{w w^{\prime}-w / 2} / \sqrt{m_{f}}$ (this goes to 0 ) and $c_{n}^{\prime}=$ $x_{\gamma_{n}} \sqrt{\gamma_{n}} / \sqrt{n m_{f}} \sim n^{w w^{\prime}+w / 2-1 / 2} / \sqrt{m_{f}}$ (this goes to 0 ). Then we have established that (11) and (12) in Corollary 1 hold under $\mathrm{P}_{n f}$, with the supremums deleted for $j=1, G=F_{C_{\text {bis }}^{\mathrm{CT}}, 0}$, $\boldsymbol{Z}_{n}=\boldsymbol{Z}^{(n)}$, and $b_{n}=d_{n} \geq\left(2 \exp \left(-\gamma^{\prime \prime} n^{w w^{\prime}}\right)+\exp \left(-n^{C / 2}\right)\right) / a_{n}$. For example, we can take $b_{n}=d_{n}=\exp \left(-n^{\min \left(w w^{\top} / 2, C / 3\right)}\right)$.

Since these bounds are valid for any starting point $s_{0}$ and any $j,(11)$ and (12) in Corollary 1 hold when the supremums are re-established, by Markovianity of the sequence $\left(\boldsymbol{Z}^{n}(j / \sqrt{n})\right.$, $j \geq 0$ ). The assumptions of Corollary 1 are therefore satisfied. The conclusions of Corollary 1 entail those of the present lemma.

\subsection{Local representation of navigations using directed navigations}

The aim of this section is to represent locally around a point $s$ the first stages of navigation (cross or straight) under the homogeneous PPP $\mathrm{P}_{n f(s)}$ using the first stages of directed navigation.

3.9.1. Local representation of straight navigation using directed navigation. Here we compare the stages of ST and DT navigation. The same results also hold for SY navigation.

Lemma 5. Let $S \in \Omega(n, C)$, and let $(s, t) \in \mathscr{D}^{\prime}[a]$ be such that $|t-s| \geq n^{B-1 / 2}$. The ST-decision domains of the first $\gamma_{n} \sim n^{w}$ stages are $\mathrm{ST}(s, t)$-simple, if $C+w<B$ for large enough $n$.

Proof. If $|t-s| \geq n^{B-1 / 2}$, since $S \in \Omega(n, C)$, we have $\left|t-s_{i}\right| \geq n^{B-1 / 2}-\gamma_{n} n^{C-1 / 2} \geq$ $n^{B-1 / 2} / 2$ and $\left|s-s_{i}\right| \leq \gamma_{n} n^{C-1 / 2}$ for $i \leq \gamma_{n}$ (for large enough $n$ ). Therefore, for $i \leq \gamma_{n}$, $\left|\arg \left(t-s_{i}\right)-\arg (t-s)\right|$ is bounded above by $O\left(n^{C+w-B}\right)$. The angle between the bisecting lines of the decision domains go to 0 uniformly; then the decision domains are nonintersecting.

Lemma 6. Let $(s, t) \in \mathscr{D}^{\prime}[a]$ be such that $|t-s| \geq n^{B-1 / 2}$ and $\gamma_{n} \sim n^{w}$. Assume that $C+$ $w<B$. For any $\varepsilon>0$ and any $x>0$, under $\mathrm{P}_{n x}$, we have the vectors $\Delta^{\mathrm{ST}}\left[s, t, \gamma_{n}\right] \mathbf{1}_{\left[0, n^{C-1 / 2}\right]} \times$ $\left(\max \left|\Delta^{\mathrm{ST}}\left[s, t, \gamma_{n}\right]\right|\right)$ and

$$
\left.\left(\mathrm{e}^{\mathrm{i} \arg \left(t-s_{j-1}\right)} \Delta^{\mathrm{DT}}(s, j), j=1, \ldots, \gamma_{n}\right) \mathbf{1}_{[0, n}{ }^{C-1 / 2}\right]\left(\max \left|\Delta^{\mathrm{DT}}\left[s, \gamma_{n}\right]\right|\right),
$$

where $s_{j}=\sum_{i=0}^{j-1} \Delta^{\mathrm{DT}}(s, i)+s$, have the same distribution when $n$ is large enough. 
Note that, under $\mathrm{P}_{n x}$, the stages $\Delta^{\mathrm{DT}}\left[s, \gamma_{n}\right]$ are i.i.d. whereas the stages $\Delta^{\mathrm{ST}}\left[s, \gamma_{n}\right]$ are not.

Proof of Lemma 6. This is a simple consequence of the fact that, under the hypothesis, the decision domains under ST or DT are simple; then the distribution of the stages in both cases are given by the same computations (based on the areas of triangles).

3.9.2. Local representation of cross navigation using directed navigation. Here we treat CT navigation, but $\mathrm{CY}$ navigation can be treated similarly. For any two points $(s, t) \in \mathscr{D}$, let $K_{s, t}:=\left\lfloor\arg (t-s) / \theta+\frac{1}{2}\right\rfloor$ be an integer $\kappa$ such that $t \in \operatorname{Sect}[\kappa, s]$.

Lemma 7. Let $\theta \leq \pi / 3$, let $S \in \Omega(n, C)$, and let $(s, t) \in \mathscr{D}^{\prime}[a]$ be such that $|t-s| \geq n^{B-1 / 2}$. The CT-decision domains for the first $\gamma_{n} \sim n^{w}$ stages are CT $(s, t)$-simple, if $C+w<B$ for large $n$.

Proof. Two cases have to be considered.

Case 1. When $t$ is far from $\operatorname{Cross}(s), K_{s_{i}, t}$ stays constant for small values of $i$. Hence, the bisecting line of the decision domains of the traveller are parallel, and so the domains do not intersect.

Case 2. When $t$ is close to $\operatorname{Cross}(s)$ but $|s-t| \geq n^{B-1 / 2}$, during the first $n^{w}$ stages, $t$ remains close to $\operatorname{Cross}\left(s_{i}\right)$, but $\left|t-s_{i}\right| \geq|t-s|-n^{w+C}-1 / 2 \geq n^{-1 / 2}\left(n^{B}-n^{C+w}\right)$. Therefore, $K_{s_{i}, t}$ can take two values, $K_{s, t}$ and $K_{s, t} \pm 1, \bmod p_{\theta}$, depending on the position of $t$ with respect to $\operatorname{Cross}(s)$. Therefore, the bisecting lines of the decision domains have two possible directions. The angle between these possible directions being $\theta \leq \pi / 3$; the decision domains are nonintersecting.

We now give a representation of the increments of the cross navigation, which is immediate since, as for Lemma 6, it simply relies on the fact that some triangles have the same area (and in Lemma 7).

Lemma 8. Let $(s, t) \in \mathscr{D}^{\prime}[a]$ be such that $|t-s| \geq n^{B-1 / 2}$. Let $\gamma_{n} \sim n^{w}$ and $C+w<B$. For any $x>0$, under $\mathrm{P}_{n x}$, we have the variables

$$
\left(\mathrm{e}^{\mathrm{i} \theta \kappa_{j}} \Delta^{\mathrm{DT}}(s, j), j=1, \ldots, \gamma_{n}\right) \mathbf{1}_{\left[0, n^{C-1 / 2}\right]}\left(\max \left|\Delta^{\mathrm{DT}}\left[s, \gamma_{n}\right]\right|\right)
$$

and $\Delta^{\mathrm{CT}}\left[s, t, \gamma_{n}\right] \mathbf{1}_{[0, n}^{C-1 / 2]}\left(\max \left|\Delta^{\mathrm{CT}}\left[s, t, \gamma_{n}\right]\right|\right)$, where the $\kappa_{j}=K_{s+\sum_{l=1}^{j-1} \Delta^{\mathrm{DT}}(s, l), t}$ have the same law.

Let us examine the consequences of Lemma 8. As in the proof of Lemma 7, two cases occur.

Case 1: when $t$ is far from $\operatorname{Cross}(s)$. Assume that we are in $\Omega(n, C)$. Assume further that $d(t, \operatorname{Cross}(s)) \geq n^{w+C-1 / 2}$, i.e. the distance between the point $t$ and the set $\operatorname{Cross}(s)$ is greater than $n^{w+C-1 / 2}$. In this case, $K_{s_{i}, t}$ is equal to $K_{s, t}$ for $i \leq n^{w}$. Therefore, the property of Lemma 8 can be rewritten as

$$
\begin{aligned}
& \left.\Delta^{\mathrm{CT}}\left[s, t, \gamma_{n}\right] \mathbf{1}_{[0, n}{ }^{C-1 / 2}\right] \\
& \left.\quad=\mathrm{e}^{\mathrm{i} \theta K_{s, t}} \Delta^{\mathrm{DT}}\left[s, \gamma_{n}\right] \mathbf{1}_{[0, n} \Delta^{\mathrm{CT}-1 / 2}\right]\left(\max \left|\Delta^{\mathrm{DT}}\left[s, \gamma_{n}\right]\right|\right) ;
\end{aligned}
$$

thus, under these conditions, CT navigation coincides with DT navigation with direction $\theta K_{s, t}$.

Case 2: when $t$ is close to $\operatorname{Cross}(s)$. Assume now that $d(t, \operatorname{Cross}(s)) \leq n^{w+C-1 / 2}$, but $|t-s| \geq n^{B-1 / 2}$. There exists a unique $k$ such that $d\left(t, \mathrm{HL}_{k}(s)\right) \leq n^{w+C-1 / 2}$ for large enough $n$ (since $B>w+C$ ). Consider the line $\boldsymbol{D}$ parallel to $\mathrm{HL}_{k}(s)$ passing via $t$ ( $\boldsymbol{D}$ is included in $\operatorname{Cross}(t)$ if $p_{\theta}$ is even). We then have $d(\boldsymbol{D}, s) \leq n^{w+C-1 / 2}$. 


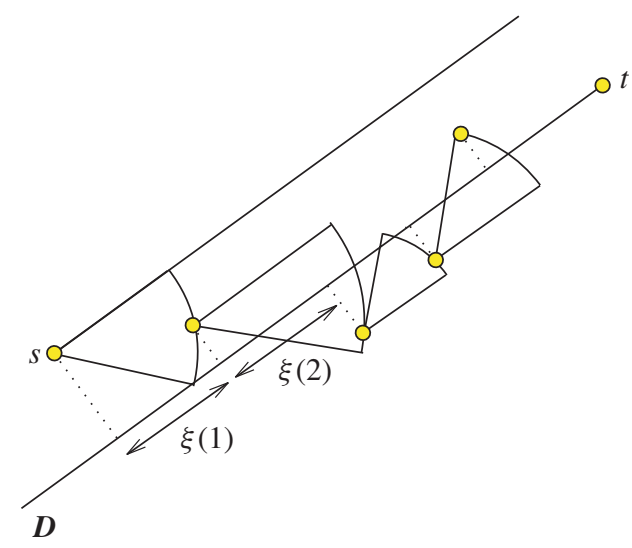

FiguRE 9: Progression of the traveller near the line $\boldsymbol{D}$. Note that the decision sectors always have a side parallel to $\boldsymbol{D}$.

The following statements hold.

(i) For any $i \leq n^{w}, d\left(\boldsymbol{D}, s_{i}\right) \leq n^{w+C-1 / 2}$. In words, if the traveller is close to $\boldsymbol{D}$ at some time, they stay close to it afterward. The reason is simple and follows from statement (ii) below: the decision domains of the traveller have a border parallel to $\boldsymbol{D}$ (see Figure 9).

(ii) The decision domains of the traveller for these $n^{w}$ steps have a border parallel to $\boldsymbol{D}$, and the other border of course presents an angle $\theta$ with $\boldsymbol{D}$. Therefore, the orthogonal projections of the stages on $\boldsymbol{D}$ have the same distribution (see Figure 9).

\section{Proofs of the theorems}

The proofs of our theorems are decomposed into several parts.

(a) First, we prove that, for any fixed $(s, t)$ and any navigation $\boldsymbol{X}$, the function $\operatorname{Pos}_{s, t}^{\boldsymbol{X}}$ admits a limit (specified in the different theorems of the paper). The different costs associated with the path (for instance, its length, which appears as a cost that cannot be handled without knowing the position of the traveller) are treated afterward.

(b) The 'one-trajectory' result is then extended to all trajectories in several steps. First, the results from (a) give, with some probability, bounds that allow us to handle a polynomial number of trajectories at once (for starting and ending points in a grid). Then the paths between other points are treated by comparison with these trajectories (see Section 4.3).

\subsection{One-trajectory result}

4.1.1. Straight navigation. For any function $g$ and any set $L$, the hitting time of $L$ by $g$ is $\tau[g](L):=\inf \{x, g(x) \in L\}$. For example,

$$
\tau\left[\operatorname{Pos}_{s, t}^{\infty, C_{\text {bis }}^{X}}\right](\{t\})=\text { Time }_{s, t}^{C_{\text {bis }}^{X}} .
$$

We introduce a uniform big O notation: let $\left(g_{n}\right)$ be a sequence of functions $g_{n}: A \rightarrow \mathbb{R}$, and let $\left(c_{n}\right)$ be a sequence of real numbers. The notation $g_{n}=O_{A}\left(c_{n}\right)$ means that $\sup _{y \in A} g_{n}(y)=$ $O\left(c_{n}\right)$. 
Theorem 6. Let $\boldsymbol{X}=\mathrm{SY}$ and $\theta<\pi / 2$, or $\boldsymbol{X}=\mathrm{ST}$ and $\theta \leq \pi / 2$. For any $\alpha \in\left(0, \frac{1}{8}\right)$, there exists $c=c(\alpha)$ such that

$$
\mathrm{P}_{n f}\left(\sup _{x \leq \operatorname{Time}_{s, t}} \boldsymbol{C}_{\text {bis }}^{X}\left|\operatorname{Pos}_{s, t}^{X}(x \sqrt{n})-\operatorname{Pos}_{s, t}^{\infty, C_{\text {bis }}^{X}}(x)\right| \geq n^{-\alpha}\right)=O_{D^{\prime}[a]}\left(\exp \left(-n^{c}\right)\right) .
$$

Moreover, there exists $d>0$ such that, for large enough $n$,

$$
\mathrm{P}_{n f}\left(\left|\frac{\mathrm{Nb}^{X}(s, t)}{\sqrt{n}}-\operatorname{Time}_{s, t}^{C_{\text {bis }}^{X}}\right| \geq d n^{-\alpha}\right)=O_{D^{\prime}[a]}\left(\exp \left(-n^{c}\right)\right) .
$$

Note that the second assertion of Theorem 6 is not a direct consequence of the first assertion since, when $\left\|g_{n}-g\right\|_{\infty} \rightarrow 0$, it may happen that $\tau\left[g_{n}\right](A) \not \rightarrow \tau[g](A)$ for some set $A$. Note also that, for $\boldsymbol{X} \in\{\mathrm{ST}, \mathrm{SY}\}, \mathrm{Time}_{s, t}^{C_{\text {bis }}^{X}}$ is implicitly known by

$$
\operatorname{Time}_{s, t}^{\boldsymbol{C}_{\text {bis }}^{X}}:=\inf \left\{x, \int_{0}^{x} \frac{\mathrm{e}^{\mathrm{i} \arg (t-s)} \boldsymbol{C}_{\mathrm{bis}}^{\boldsymbol{X}}}{\sqrt{f\left(s+\mathrm{e}^{\mathrm{i} \arg (t-s) u}\right)}} \mathrm{d} u=t-s\right\} .
$$

If $f=c$ is constant, this simplifies and we obtain

$$
\text { Time }_{s, t}^{C_{\text {bis }}^{X}}=\frac{|t-s| \sqrt{c}}{C_{\text {bis }}^{X}} .
$$

Proof of Theorem 6. We give the proof in the case $\boldsymbol{X}=\mathrm{ST}$; the $\boldsymbol{X}=\mathrm{SY}$ case is similar. For short, write $\bar{Z}_{s}^{(n)}(x)$ instead of $\operatorname{Pos}_{s, t}^{\mathrm{ST}}(x \sqrt{n})$. For $B \in\left(0, \frac{1}{4}\right)$ and $\varepsilon>0$, consider the set $\Omega_{n, \varepsilon}$ defined in Section 3.5. By Lemmas 2 and 3, $\mathrm{P}_{n f}\left(C \Omega_{n, \varepsilon}\right) \leq \exp \left(-n^{c_{1}}\right)$ for some $c_{1}>0$ and large enough $n$. Let us assume that $S \in \Omega_{n, \varepsilon}$. Consider $T_{s, t}^{n}:=\inf \left\{x,\left|\bar{Z}_{S}^{(n)}(x)-t\right|<n^{B-1 / 2}\right\}$, the hitting time of $B\left(t, n^{B-1 / 2}\right)$ by $\bar{Z}_{s}^{(n)}$. By Proposition $1, T_{s, t}^{n}<+\infty$ a.s., and, for any $x \geq T_{s, t}^{n}$,

$$
\left|\overline{\mathbf{Z}}_{x}^{(n)}(x)-t\right| \leq n^{B-1 / 2} \text {. }
$$

For $S \in \Omega_{n, \varepsilon}$, the total number of stops inside $B\left(t, n^{B-1 / 2}\right)$ is at most $n^{(2+\varepsilon) B}$, which corresponds on the process $\overline{\boldsymbol{Z}}^{(n)}$ to a negligible time interval $n^{(2+\varepsilon) B-1 / 2}=o(1)$ if $(2+\varepsilon) B<\frac{1}{2}$. The space fluctuations of these last stages are at most of order $n^{B-1 / 2}$, and so they are negligible when

$$
B-\frac{1}{2}<-\alpha .
$$

The control of the position of the traveller along the rest of the trajectory will be established using Corollary 1, together with elements from the proof of Theorem 6, and Lemma 6.

Assume that $|s-t|>n^{B-1 / 2}, w>0, \gamma_{n}=n^{w}$, and $y_{n}=x_{\gamma_{n}} \sqrt{\gamma_{n}} / \sqrt{n m_{f}}$ for $x_{n} \sim$ $n^{w^{\prime}}, w^{\prime} \in\left(0, \frac{1}{2}\right)$, as in the proof of Theorem 5. By Proposition 4 we know that, for an ST(s,t)simple set $\Theta$,

$$
\mathrm{P}_{n f}\left(\Delta^{\mathrm{ST}}\left[s, t, \gamma_{n}\right] \in \Theta\right) \leq \exp \left(-n^{C / 2}\right)+2 \mathrm{P}_{n f(s)}\left(\Delta^{\mathrm{ST}}\left[s, t, \gamma_{n}\right] \in \Theta\right) .
$$

From now on we work under $\mathrm{P}_{n f(s)}$. Set, for fixed $(s, t)$,

$$
\begin{gathered}
\Psi: \mathbb{C}^{\gamma_{n}} \rightarrow \mathbb{C}^{\gamma_{n}}, \\
\lambda:=\left(\lambda_{1}, \ldots, \lambda_{\gamma_{n}}\right) \mapsto\left(\mathrm{e}^{\mathrm{i} \arg \left(t-s_{k-1}(\lambda)\right)} \lambda_{k}, k=1, \ldots, \gamma_{n}\right) \mathbf{1}_{\left[0, n^{C-1 / 2}\right]}\left(\max _{l \in \llbracket 1, \gamma_{n} \rrbracket}\left|\lambda_{l}\right|\right),
\end{gathered}
$$


where $s_{k-1}(\lambda)=s+\sum_{i=1}^{k-1} \lambda_{i}$. Hence, Lemma 6 says that, under $\mathrm{P}_{n f(s)}$,

$$
\begin{aligned}
& \Psi\left(\Delta^{\mathrm{DT}}\left[s, \gamma_{n}\right]\right) \mathbf{1}_{[0, n}{ }_{[-1 / 2]}\left(\max \Delta^{\mathrm{DT}}\left[s, \gamma_{n}\right]\right) \\
& \left.\quad \stackrel{\mathrm{D}}{=} \Delta^{\mathrm{ST}}\left[s, t, \gamma_{n}\right] \mathbf{1}_{[0, n}{ }^{C-1 / 2}\right] \\
&
\end{aligned}
$$

For $\Theta_{n}^{(1)}$ given in (18), define

$$
\Theta_{n}^{(2)}:=\Theta_{n}^{(1)} \cap\left\{\left(\lambda_{1}, \ldots, \lambda \gamma_{n}\right) \in \mathbb{C}^{\gamma_{n}}, \max _{l \in \llbracket 1, \gamma_{n} \rrbracket}\left|\lambda_{l}\right|<n^{C-1 / 2}\right\} .
$$

By Lemmas 2 and 4, for some $\gamma^{\prime \prime}$ and large enough $n$,

$$
\mathrm{P}_{n f(s)}\left(\Delta^{\mathrm{DT}}\left[s, \gamma_{n}\right] \in \Theta_{n}^{(2)}\right) \geq 1-\exp \left(-\gamma^{\prime \prime} n^{w w^{\prime}}\right)-\exp \left(-n^{C / 2}\right) .
$$

If $\Delta^{\mathrm{DT}}\left[s, \gamma_{n}\right] \in \Theta_{n}^{(2)}$ then it is also in $\Theta_{n}^{(1)}$ and so most of the equalities or set inclusions of the proof of Lemma 4 can be recycled here, starting from

$$
\begin{aligned}
& \left\{\Delta^{\mathrm{DT}}\left[s_{0}, \gamma_{n}\right] \in \Theta_{n}^{(2)}\right\} \\
& \quad=\left\{\max _{l \in \llbracket 1, \gamma_{n} \rrbracket}\left|\sum_{j=1}^{l}\left(\Delta^{\mathrm{DT}}\left(s_{0}, j\right)-\frac{\boldsymbol{C}_{\text {bis }}^{\mathrm{CT}}}{\sqrt{n f\left(s_{0}\right)}}\right)\right| \leq y_{n}, \max \Delta^{\mathrm{DT}}\left[s_{0}, \gamma_{n}\right]<n^{C-1 / 2}\right\} .
\end{aligned}
$$

For $\Delta^{\mathrm{DT}}\left[s_{0}, \gamma_{n}\right] \in \Theta_{n}^{(2)}$ and $\boldsymbol{Z}^{(n)}$ defined as in the proof of Lemma 4 , since $\left|\mathrm{e}^{\mathrm{i} \arg (t-s)}\right|=1$,

$$
\begin{gathered}
\left|\mathrm{e}^{\mathrm{i} \arg (t-s)} \frac{\boldsymbol{Z}_{s_{0}}^{(n)}\left(a_{n}\right)-s_{0}}{a_{n}}-\mathrm{e}^{\mathrm{i} \arg (t-s)} F_{\boldsymbol{C}_{\mathrm{bis}}^{\mathrm{CT}}, 0}\left(\boldsymbol{Z}_{s_{0}}^{(n)}(0)\right)\right| \leq \frac{y_{n}}{a_{n}}, \\
\sup _{x \in\left[0, a_{n}\right]}\left|\mathrm{e}^{\mathrm{i} \arg (t-s)}\left(\boldsymbol{Z}_{s_{0}}^{(n)}(x)-\boldsymbol{Z}_{s_{0}}^{(n)}(0)\right)-(x-0) \mathrm{e}^{\mathrm{i} \arg (t-s)} F_{\boldsymbol{C}_{\text {bi }}^{\mathrm{CT}}, 0}\left(\boldsymbol{Z}_{s_{0}}^{(n)}(0)\right)\right| \leq y_{n} .
\end{gathered}
$$

Consider now the increments $\widehat{\Delta}\left[s, t, \gamma_{n}\right]:=\Psi\left(\Delta^{\mathrm{DT}}\left[s, \gamma_{n}\right]\right)$. Using (22) and (23), for any Borelian $\Theta$,

$$
\left|\mathrm{P}_{n f(s)}\left(\widehat{\Delta}\left[s, t, \gamma_{n}\right] \in \Theta\right)-\mathrm{P}_{n f(s)}\left(\Delta^{\mathrm{ST}}\left[s, t, \gamma_{n}\right] \in \Theta\right)\right| \leq \exp \left(-\gamma^{\prime \prime} n^{w w^{\prime}}\right)+\exp \left(-n^{C / 2}\right) .
$$

Up to this exponentially small probability, we may work with $\widehat{\Delta}\left[s, t, \gamma_{n}\right]$ instead of $\Delta^{\mathrm{ST}}\left[s, t, \gamma_{n}\right]$. For $\Delta^{\mathrm{DT}}\left[s, \gamma_{n}\right] \in \Theta_{n}^{(2)}$ and $k \leq \gamma_{n}$, let us bound

$$
d:=\left|\sum_{j=1}^{k}\left(\widehat{\Delta}(s, t, j)-\mathrm{e}^{\mathrm{i} \arg (t-s)} \Delta^{\mathrm{DT}}(j)\right)\right| .
$$

Writing $S_{j}=\sum_{m=1}^{j} \Delta^{\mathrm{DT}}(j)$

$$
\begin{aligned}
d & =\left|\sum_{j=1}^{k} \mathrm{e}^{\mathrm{i} \arg \left(t-S_{j-1}\right)} \Delta^{\mathrm{DT}}(j)-\mathrm{e}^{\mathrm{i} \arg (t-s)} \Delta^{\mathrm{DT}}(j)\right| \\
& \leq \max \left\{\left|\mathrm{e}^{\mathrm{i} \arg \left(t-S_{j-1}\right)}-\mathrm{e}^{\mathrm{i} \arg (t-s)}\right|, j \in \llbracket 0, k \rrbracket\right\} \sum_{j=1}^{k}\left|\Delta^{\mathrm{DT}}(j)\right| .
\end{aligned}
$$


Since $\Delta^{\mathrm{DT}}\left[s, \gamma_{n}\right] \in \Theta_{n}^{(2)}$, the second term on the right-hand side of (26) is bounded by $k C_{\text {bis }}^{\mathrm{CT}} / \sqrt{n f(s)}+y_{n}$, which is smaller than $O_{D^{\prime}[a]}\left(a_{n}+y_{n}\right)=O_{D^{\prime}[a]}\left(n^{w-1 / 2}\right)$ (since $y_{n}=$ $\left.o\left(a_{n}\right)\right)$. Now, to control the maximum, we compare $\arg \left(t-S_{j}\right)$ with $\arg (t-s)$. For $\Delta^{\mathrm{DT}}\left[s, \gamma_{n}\right] \in$ $\Theta_{n}^{(2)}$ and $j \in \llbracket 0, \gamma_{n} \rrbracket$,

$$
\left|S_{j}-s\right| \leq \frac{C_{\mathrm{bis}}^{\mathrm{CT}}}{m_{f}} \gamma_{n} n^{-1 / 2}+y_{n}=O_{D^{\prime}[a]}\left(n^{w-1 / 2}+y_{n}\right)=O_{D^{\prime}[a]}\left(n^{w-1 / 2}\right)
$$

(since $y_{n}=o\left(a_{n}\right)$ ), and so

$$
\left|t-S_{j}\right| \geq n^{B-1 / 2}-\left|s-S_{j}\right| \geq \frac{1}{2} n^{B-1 / 2}
$$

for large enough $n$ since $w<B$ (uniformly in $s \in \mathscr{D}(a)$ ). Using the fact that $\left|\mathrm{e}^{\mathrm{i} a}-1\right|=O(|a|)$, we obtain

$$
\max _{j \in \llbracket 0, \gamma_{n} \rrbracket}\left|\mathrm{e}^{\mathrm{i}\left(\arg \left(t-S_{j-1}(\lambda)\right)-\arg (t-s)\right)}-1\right|=O_{\mathscr{D}^{\prime}[a]}\left(\frac{y_{n}}{n^{B-1 / 2}}\right) .
$$

(The tangent of the angle $\angle\left(S_{j-1}, t, s\right)$ is $O\left(y_{n} /\left|t-S_{j}\right|\right)$.) Then,

$$
d=O_{D^{\prime}[a]}\left(n^{w w^{\prime}+w / 2-B+w-1 / 2}\right)=O_{D^{\prime}[a]}\left(n^{w w^{\prime}+3 w / 2-B-1 / 2}\right) .
$$

Set $\widehat{Z}_{s}^{(n)}(j / \sqrt{n})=s+\sum_{l=1}^{j} \widehat{\Delta}(s, t, l)$. Again, for any Borelian set $\Theta$,

$$
\left|\mathrm{P}_{n f(s)}\left(\widehat{\boldsymbol{Z}}_{s}^{(n)} \in \Theta\right)-\mathrm{P}_{n f(s)}\left(\overline{\boldsymbol{Z}}_{s}^{(n)} \in \Theta\right)\right| \leq \exp \left(-\gamma^{\prime \prime} n^{w w^{\prime}}\right)+\exp \left(-n^{C / 2}\right) .
$$

We have

$$
\sup _{x \in\left[0, a_{n}\right]}\left|\widehat{\boldsymbol{Z}}_{s}^{(n)}(x)-\mathrm{e}^{\mathrm{i} \arg (t-s)} \boldsymbol{Z}^{(n)}(x)\right| \leq d=O_{D^{\prime}[a]}\left(n^{w w^{\prime}+3 w / 2-B-1 / 2}\right) .
$$

Hence, using (24) and (25), if $\Delta^{\mathrm{DT}}\left[s, \gamma_{n}\right] \in \Theta_{n}^{(2)}$ then

$$
\left\{\left|\frac{\widehat{\boldsymbol{Z}}_{s_{0}}^{(n)}\left(a_{n}\right)-s_{0}}{a_{n}}-\mathrm{e}^{\mathrm{i} \arg (t-s)} F_{\boldsymbol{C}_{\text {bis }}^{\mathrm{CT}}, 0}\left(\widehat{\boldsymbol{Z}}_{s_{0}}^{(n)}(0)\right)\right| \leq \frac{y_{n}}{a_{n}}+\frac{d}{a_{n}}\right\}
$$

and

$$
\left\{\sup _{x \in\left[0, a_{n}\right]}\left|\left(\widehat{\boldsymbol{Z}}_{s_{0}}^{(n)}(x)-\widehat{\boldsymbol{Z}}_{s_{0}}^{(n)}(0)\right)-(x-0) \mathrm{e}^{\mathrm{i} \arg (t-s)} F_{\boldsymbol{C}_{\text {bis }}^{\mathrm{CT}}, 0}\left(\widehat{\boldsymbol{Z}}_{s_{0}}^{(n)}(0)\right)\right| \leq y_{n}+d\right\} .
$$

By (23), this occurs with a probability exponentially close to 1 under $\mathrm{P}_{n f(s)}$; this is also true for $\overline{\boldsymbol{Z}}^{(n)}$ under $\mathrm{P}_{n f(s)}$ by (27), and for $\overline{\boldsymbol{Z}}^{(n)}$ under $\mathrm{P}_{n f}$ by (21).

We are now in a position to use Corollary 1 on the process $\overline{\boldsymbol{Z}}^{(n)}$ under $\mathrm{P}_{n f}$. The corresponding value of $\max \left\{a_{n}, c_{n}, c_{n}^{\prime}\right\}$ is $M_{n}:=\max \left\{n^{w-1 / 2},\left(y_{n}+O_{\mathcal{D}^{\prime}[a]}\left(n^{w w^{\prime}+3 w / 2-B-1 / 2}\right)\right) / a_{n}\right\}=$ $\max \left\{n^{w-1 / 2}, O_{D^{\prime}[a]}\left(n^{w w^{\prime}-w / 2}\right), O_{D^{\prime}[a]}\left(n^{w w^{\prime}+w / 2-B}\right)\right\}$, and the probabilities $b_{n}$ and $d_{n}$ are smaller than $\exp \left(-n^{c}\right)$ for some $c>0$ and large enough $n$. We want $M_{n}$ to be as small as possible. For this, we choose $w^{\prime}$ close to 0 , and since $B<\frac{1}{4}$, the maximum is obtained by taking $w=B^{-}$. In this case, $\max \left\{a_{n}, c_{n}, c_{n}^{\prime}\right\}=n^{-B / 2^{-}}$. Now, the conclusion of the theorem holds if $-B / 2^{-}<-\alpha$ and $B-\frac{1}{2}<-\alpha$. Hence, $\alpha$ must be chosen in $\left(0, \frac{1}{8}\right)$ for the existence of $B, \varepsilon^{\prime}$, and $w$ satisfying all the requirements of the present proof and at any time less than or equal to Time $_{s, t}^{C_{\text {bis }}^{X}}$ as long as $\operatorname{Pos}_{s, t}^{X}(x \sqrt{n})$ is outside $B\left(t, n^{B-1 / 2}\right)$. By the above, at time $\operatorname{Time}_{s, t} \boldsymbol{C}_{\text {bis }}^{X}$, the 
traveller is in $B\left(t, n^{-\alpha}\right)$ with probability $1-\exp \left(-n^{c}\right)$; therefore, by Proposition 1 , after this time the traveller will come closer to $\{t\}$ at each stage. This implies the first assertion of the theorem.

We now consider the proof of the second assertion. It remains to show that the number of steps needed for the traveller to reach the target once in $B\left(t, n^{-\alpha}\right)$ is negligible before $\sqrt{n}$. From what is said below (20), we need to only control the number of stages necessary to enter $B\left(t, n^{B-1 / 2}\right)$ from time $\operatorname{Time}_{s, t} \boldsymbol{C}_{\text {bis }}^{X}$, at which time we know that the traveller is in $B\left(t, n^{-\alpha}\right)$. The argument below (20) will allow us to see that at most $d n^{-\alpha+1 / 2}$ steps for a constant $d$ will be needed, with probability exponentially close to 1 . For this, we observe that Lemma 6 can still be used, as well as the sets $\Theta_{n}^{(2)}$ defined above. The sum of the length of the increments after $c_{n}$ steps (once in $B\left(t, n^{-\alpha}\right)$, with $c_{n} \geq \gamma_{n}$, is at least $d c_{n} n^{-1 / 2} \pm c_{n} y_{n} / \gamma_{n}$ for a constant $d$, i.e. $d c_{n} n^{-1 / 2}$ to first order) with probability exponentially close to 1 . Hence, by Proposition 1(iv), the number of steps needed to traverse a distance at most $n^{-\alpha}$ is at most $d n^{-\alpha+1 / 2}$ for a constant $d$.

\subsubsection{Cross navigation.}

Theorem 7. Let $\boldsymbol{X} \in\{\mathrm{CY}, \mathrm{CT}\}$ and $\theta \leq \pi / 3$. For any $\alpha \in\left(0, \frac{1}{8}\right)$, there exists $c=c(\alpha)$ such that

$$
\mathrm{P}_{n f}\left(\sup _{x \in\left[0, \tau_{s, t}^{X}\right]}\left|\operatorname{Pos}_{s, t}^{X}(x \sqrt{n})-\operatorname{Pos}_{s, t}^{\infty, C_{\text {bis }}^{X}, C_{\text {bor }}^{X}}(x)\right| \geq n^{-\alpha}\right)=O_{D^{\prime}[a]}\left(\exp \left(-n^{c}\right)\right),
$$

where $\tau_{s, t}^{X}=\operatorname{Time}_{s, I(s, t)}^{C_{\mathrm{bis}}^{X}}+\operatorname{Time}_{I(s, t), t}^{C_{\mathrm{bor}}^{X}}$. Moreover, there exists $d>0$ such that, for large enough $n$,

$$
\mathrm{P}_{n f}\left(\left|\frac{\mathrm{Nb}_{s, t}^{X}}{\sqrt{n}}-\tau_{s, t}^{X}\right| \geq d n^{-\alpha}\right)=O_{D^{\prime}[a]}\left(\exp \left(-n^{c}\right)\right) .
$$

Again, for $\boldsymbol{X} \in\{\mathrm{CT}, \mathrm{CY}\}, \tau_{s, t}^{X}$ is implicitly known since

$$
\begin{aligned}
& \operatorname{Time}_{s, I(s, t)}^{\boldsymbol{C}_{\text {bis }}^{X}}:=\inf \left\{x, \int_{0}^{x} \frac{\mathrm{e}^{\mathrm{i} \arg (I(s, t)-s)} \boldsymbol{C}_{\text {bis }}^{X}}{\sqrt{f\left(s+\mathrm{e}^{\mathrm{i} \arg (I(s, t)-s) u}\right)}} \mathrm{d} u=I(s, t)-s\right\}, \\
& \text { Time }_{I(s, t), t}^{\boldsymbol{C}_{\text {bor }}^{X}}:=\inf \left\{x, \int_{0}^{x} \frac{\mathrm{e}^{\mathrm{i} \arg (t-I(s, t))} \boldsymbol{C}_{\text {bor }}^{X}}{\sqrt{f\left(I(s, t)+\mathrm{e}^{\mathrm{i} \arg (t-I(s, t)) u}\right)}} \mathrm{d} u=t-I(s, t)\right\} .
\end{aligned}
$$

If $f=c$ is constant, this gives

$$
\tau_{s, t}^{X}=\sqrt{c}\left(\frac{|I(s, t)-s|}{C_{\mathrm{bis}}^{X}}+\frac{|t-I(s, t)|}{C_{\mathrm{bor}}^{X}}\right) .
$$

Proof of Theorem 7. We consider only the $\boldsymbol{X}=\mathrm{CT}$ case, since the $\boldsymbol{X}=\mathrm{CY}$ case is similar.

Recall the contents of Lemma 8, and of the last two paragraph of Sections 3.9. The start of the proof is the same as that of Theorem 6, using also Proposition 1, and again we consider only the case where $|s-t|>n^{B-1 / 2}$ and the portion of the traveller's trajectory that is outside the ball $B\left(t, n^{B-1 / 2}\right)$, as in the proof of Theorem 6. Consider the set $\Omega_{n, \varepsilon}$ (defined in Section 3.5). When $t$ is far from $\operatorname{Cross}(t)$, that is, if $d\left(t, \operatorname{Cross}\left(s_{0}\right)\right) \geq n^{w+C-1 / 2}$, by (19), CT navigation coincides exactly with DT navigation with direction $\theta K_{s, t}$ for $n^{w}$ stages, provided that $S \in \Omega_{n, \varepsilon}$ (since only $n^{w}$ stages of size at most Navmax $[\theta]$ are concerned). Therefore, all the properties and bounds obtained for this case, particularly those in the proof of Theorem 5, hold here also. 
Hence, the traveller will stay close to $\boldsymbol{B}_{K_{s, t}}$, the bisecting line of $\operatorname{Sect}\left[K_{s, t}, s\right]$, its fluctuation around this line being larger than $n^{-\alpha}$ for $\alpha \in\left(0, \frac{1}{8}\right)$ with exponentially small probability. Therefore, the trajectory of the traveller from its starting point until a neighbourhood of $I_{s, t}$ will be the solution of $\operatorname{ODE}\left(\boldsymbol{C}_{\mathrm{bis}}^{\mathrm{CT}}, \arg \left(I_{s, t}-s\right), s_{0}\right)$.

Assume now that the traveller satisfies $d\left(t, \operatorname{Cross}\left(s_{0}\right)\right) \leq n^{w+C-1 / 2}$ (this can occur at the beginning of their trip or after some sequences of $n^{w}$ consecutive stages). Recall the considerations in the last paragraph of Section 3.9 and Figure 9. If, for some $j, d\left(t, \operatorname{Cross}\left(s_{j}\right)\right) \leq$ $n^{w+C-1 / 2}$ then this will remain true until the traveller enters the final ball $B\left(t, n^{B-1 / 2}\right)$. Let us describe more precisely what happens when $d\left(t, \operatorname{Cross}\left(s_{j}\right)\right)$ is small. Denote by $\boldsymbol{D}$ the parallel to the branch of Cross $\left(s_{i}\right)$ being close to $t$, passing via $t$. In order to control the position of the traveller, knowing that it is close to $\boldsymbol{D}$, an orthogonal projection on $\boldsymbol{D}$ is used. The progression of this projection on $\boldsymbol{D}$ measures the progress of the traveller toward $t$. We will not enter into the details, everything works with respect to the orthogonal projection on $\boldsymbol{D}$ as in the case of directed navigation, since the projection $\xi$ also has some exponential moments. Therefore, once close to $\Delta$, the movement of the traveller will be asymptotically ruled by $\operatorname{ODE}\left(\boldsymbol{C}_{\mathrm{bor}}^{\mathrm{CT}}, \arg \left(t-I_{s, t}\right), s_{0}\right)$. Now, taking into account the fact that $w+C-\frac{1}{2} \leq B-\frac{1}{2}$, the traveller will necessarily enter the ball $B\left(t, n^{B-1 / 2}\right)$, where it will make a negligible number of steps, with negligible fluctuations (see the discussion below (20)).

\subsection{Analysis of the traveller's costs}

In Section 1.4 we introduced the cost $\operatorname{Cost}_{H}^{X}(s, t):=\sum_{j=1}^{\mathrm{Nb}(s, t)} H\left(\Delta_{j}^{X}\right)$ related to the traveller's journey from $s$ to $t$, associated with an elementary cost function $H$, and $X$. If $H$ is the modulus function $H: x \mapsto|x|$ then $\operatorname{Cost}_{H}^{X}(s, t)=\left|\operatorname{Path}^{X}(s, t)\right|$, and if $H: x \mapsto 1$ then $\operatorname{Cost}_{H}^{X}(s, t)=\mathrm{Nb}(s, t)$, as already discussed in Theorems 6 and 7. In the sequel we consider only the case $H_{g}: x \mapsto|x|^{g}$ for some $g \geq 0$. Other functions could certainly be studied following the same steps, but the present case covers the applications we have (discussions around the cases $g \in[2,4]$ appear in [14]).

Let us discuss a bit at the intuitive level. Under $\mathrm{P}_{n f}$, locally around position $s_{0}$, a stage $\Delta^{\mathrm{DT}}$ is close in distribution to $\Delta_{1}^{\mathrm{DT}} / \sqrt{n f\left(s_{0}\right)}$, a rescaled stage under $\mathrm{P}_{1}$. For a regular function $H$, $H\left(\Delta^{\mathrm{DT}}\right)$ is close to $H\left(\Delta_{1}^{\mathrm{DT}} / \sqrt{n f\left(s_{0}\right)}\right)$. Two main points have been noted so far. Another point is that at first order, under $\mathrm{P}_{n f}, H\left(\Delta^{\mathrm{DT}}\right)$ depends on the behaviour of $H$ near 0 . Functions $H$ 'that are regular near 0 ' are needed to obtain simple asymptotic behaviours. This justifies the choice of the class of functions $H_{g}$. The contribution to the cost of $H\left(\Delta^{\mathrm{DT}}\right)$ depends on the position of the traveller. Again, a differential equation appears: it is important to consider the pair

$$
\left(\left(\operatorname{Pos}^{X}(s, t, i), \operatorname{Cost}^{X}(s, t, i)\right), i=1, \ldots, k\right),
$$

where $\operatorname{Cost}^{X}(s, i)=\sum_{j=1}^{i} H\left(\Delta_{j}^{X}\right)$ since the cost cannot be studied independently of the position.

Another remark concerns the case $H: x \mapsto 1$, in which case $\operatorname{Cost}^{X}(s, j)=j$. In this case, by defining

$$
\mathrm{e}^{(n)}\left(\frac{j}{\sqrt{n}}\right):=\frac{\operatorname{Cost}(s, j)}{\sqrt{n}}=\frac{j}{\sqrt{n}} \text { for } j \geq 0
$$

and linear interpolation between the points $(j / \sqrt{n}, j \geq 0)$, on any interval $[0, \lambda],\left(\mathcal{C}^{(n)}, n \geq 0\right)$ converges uniformly to $c_{\text {sol }}:=y \mapsto y$. Therefore, the pair $\left(\mathcal{C}^{(n)}, \operatorname{Pos}_{s, t}^{X}(\cdot \sqrt{n})\right)$ converges to $\left(c_{\mathrm{sol}}, \operatorname{Pos}_{s, t}^{\infty, c}\right)$ under the same conditions as those under which $\operatorname{Pos}_{s, t}^{X}(\cdot \sqrt{n})$ converges to $\operatorname{Pos}_{s, t}^{\infty, c}$. Even if at first glance this convergence seems to entail that of $\mathrm{Nb}^{X}(s, t)$, this is not immediately 
the case, as observed in the proofs of Theorem 6 and 7, since the convergence of functions does not imply the convergence of hitting times. Here the same phenomena arise for other cost functions.

Let us now return to the case where $H=H_{g}$ for some $g>0$. By the scaling argument we have, for any $c>0, H_{g}\left(\Delta_{c}^{\boldsymbol{X}}\right) \stackrel{\mathrm{D}}{=}\left|\Delta_{1}^{\boldsymbol{X}}\right|^{g} / c^{g / 2}$, and then, further, under $\mathrm{P}_{c}$,

$$
\sum_{j=1}^{a_{n}} H_{g}\left(\Delta_{c}^{X}(j)\right) \stackrel{\mathrm{D}}{=} \sum_{j=1}^{a_{n}} \frac{\left|\Delta_{1}^{X}(j)\right|^{g}}{c^{g / 2}} .
$$

Since the random variables $\left|\Delta_{1}^{X}(j)\right|$ have exponential moments (see Section 3.3.2), so do $\left|\Delta_{1}^{X}(j)\right|^{g}$ for any $g>0$. Therefore, by the law of large numbers, for any $a_{n} \rightarrow+\infty$,

$$
\sum_{j=1}^{a_{n}} \frac{\left|\Delta_{1}^{X}(j)\right|^{g}}{a_{n}} \underset{n}{\stackrel{\text { a.s. }}{\longrightarrow}} \mathrm{E}\left(\left|\Delta_{1}^{X}(j)\right|^{g}\right),
$$

and Petrov's lemma can be used to control the deviation around the mean (see Section 3.3.2). Hence, at first order, $\sum_{j=1}^{a_{n}} H_{g}\left(\left|\Delta_{c}^{X}(j)\right|\right)$ is close to $\left(a_{n} / c^{g / 2}\right) \mathrm{E}\left(\left|\Delta_{1}^{X}(j)\right|^{g}\right)$, and this, for any $c$, including the case where $c=c_{n}$, depends on $n$. Under $\mathrm{P}_{n f}$, if the traveller is at position $s$ then $\left|\Delta_{n f}^{X}(1)\right|^{g}$ is close in distribution to $\left|\Delta_{1}^{X}\right|^{g} /(n f(s))^{g / 2}$, since $\mathrm{Nb}(s, t)$ has order $\sqrt{n}$. Set

$$
C^{(n), X}\left(\frac{j}{\sqrt{n}}\right):=\operatorname{Cost}^{X}(s, j) n^{g / 2-1 / 2} .
$$

In order to extend Theorem 5 to the pair (Pos, Cost), we need to introduce a system of ODEs. We already saw that at least up to some decompositions by parts of the trajectories, the limiting position of the traveller was the solution of $\operatorname{ODE}(\lambda, v, s)$ for some parameters $(\lambda, v)$ depending on the details of the studied navigation. For any $s_{0} \in \mathcal{D}, c_{0} \in \mathbb{R}$, and $q \in \mathbb{R}$, consider the following system:

$$
\mathrm{ODE}^{(2)}\left(\lambda, \nu, q, s_{0}, c_{0}\right):=\left\{\begin{array}{l}
\rho(0)=s_{0}, \quad \mathcal{C}(0)=c_{0}, \\
\frac{\partial \rho(x)}{\partial x}=F_{\lambda, v}(\rho(x))=\frac{\lambda \mathrm{e}^{\mathrm{i} v}}{f(\rho(x))^{1 / 2}}, \\
\frac{\partial \mathcal{C}(x)}{\partial x}=\frac{q}{f(\rho(x))^{g / 2}} .
\end{array}\right.
$$

The existence and uniqueness of a solution $(\rho, \mathcal{C})$ to this system is guaranteed by the CauchyLipschitz theorem. The function $\rho$ is $\operatorname{Sol}_{s_{0}}^{\lambda, v}$ since the conditions on $\rho$ coincide with $\operatorname{ODE}(\lambda, \nu$, $s_{0}$ ). The function $\mathcal{C}$ clearly has a simple integral representation using $\rho$ and $f$ :

$$
\mathcal{C}(y)=c_{0}+q \int_{0}^{y} \frac{\mathrm{d} x}{f(\rho(x))^{g / 2}} .
$$

Since

$$
\rho(y)=s_{0}+\int_{0}^{y} F_{\lambda, v}(\rho(x)) \mathrm{d} x=s_{0}+\lambda \mathrm{e}^{\mathrm{i} v} \int_{0}^{y} \frac{\mathrm{d} x}{f(\rho(x))^{1 / 2}}
$$

in the $g=1$ case, this immediately leads to

$$
\mathcal{C}(y)=c_{0}+q \lambda^{-1}\left(\rho(y)-s_{0}\right) \mathrm{e}^{-\mathrm{i} v} .
$$


If $g \neq 1, \rho$ and $\mathcal{C}$ are related by a linear formula only if $f$ is constant since in this case $\rho$ and $\mathcal{C}$ are linear functions.

Limits in the directed case. We explain in this case only the appearance of the limiting differential equation.

Lemma 9. Let $\boldsymbol{X}=\mathrm{DT}$ and $\theta \leq \pi$, or $\boldsymbol{X}=\mathrm{DY}$ and $\theta \leq \pi / 2$. The pair of processes $\left(\operatorname{Pos}_{s, t}^{X}(x \sqrt{n}), C^{(n), X}(x)\right)_{x \in[0, \lambda]}$ satisfies the assumption of Corollary 1 for $b_{n}=n^{w w^{\prime}-w / 2} \times$ $\left(m_{f}\right)^{-1 / 2}, c_{n}=\exp \left(-n^{\min \left(w w^{\prime} / 2, c\right)}\right), c_{n}^{\prime}=c_{n}$, and $d_{n}=b_{n}$. Therefore, it converges to the $\left.\operatorname{Pos}_{s, t}^{\infty, C_{\text {bis }}^{X}}(x), \operatorname{Cost}^{\infty, X}(x)\right)_{x \in[0, \lambda]}$ solution $\operatorname{ODE}^{(2)}\left(C_{\text {bis }}^{\boldsymbol{X}}, 0, \boldsymbol{C}_{\text {bis }}^{\boldsymbol{X}, g}, s_{0}, 0\right)$ for $\lambda \in\left[0, \lambda\left(F_{\boldsymbol{C}_{\text {bis }}, 0}\right.\right.$, $s))$, where $\boldsymbol{C}_{\mathrm{bis}}^{\boldsymbol{X}, g}=\mathrm{E}\left(\left|\Delta_{1}^{\boldsymbol{X}}\right|^{g}\right)$.

Proof. The proof uses the ideas in the proof of Theorem 5 (we will use $\Theta_{n}^{(1)}$ and $\boldsymbol{Z}^{(n)}$, as defined in its proof). Consider again the set $\Theta_{n}^{(1)}$ defined in (18), and introduce the following Borelian subset of $\mathbb{C}^{\gamma_{n}}$ :

$$
\Theta_{n}^{(4)}:=\left\{\left(\lambda_{1}, \ldots, \lambda_{\gamma_{n}}\right) \in \mathbb{C}^{\gamma_{n}}, \sup _{l \in \llbracket 1, \gamma_{n} \rrbracket}\left|\sum_{j=1}^{l}\left(n^{g / 2-1 / 2}\left|\lambda_{j}\right|^{g}-\frac{C_{\mathrm{bis}}^{T, g}}{\sqrt{n}\left(f\left(s_{0}\right)\right)^{g / 2}}\right)\right| \leq y_{n}\right\}
$$

for a sequence $\left(y_{n}\right)$. Using $\boldsymbol{C}^{(n)}\left(a_{n}\right)=c_{0}+n^{g / 2-1 / 2} \sum_{l=1}^{\gamma_{n}}\left|\Delta^{\mathrm{DT}}\left(s_{0}, l\right)\right|^{g}$, we obtain

$$
\left\{\Delta^{\mathrm{DT}}\left[s_{0}, \gamma_{n}\right] \in \Theta_{n}^{(4)}\right\}=\left\{\sup _{l \in \llbracket 1, \gamma_{n} \rrbracket}\left|\boldsymbol{C}_{s_{0}}^{(n)}\left(\frac{l}{\sqrt{n}}\right)-c_{0}-\frac{l}{\sqrt{n}} \frac{\boldsymbol{C}_{\mathrm{bis}}^{T, g}}{f\left(s_{0}\right)^{g / 2}}\right| \leq y_{n}\right\},
$$

and everything works as in the proof of Theorem 5, in particular, using rescaling (8),

$$
\begin{aligned}
\mathrm{P}_{n f\left(s_{0}\right)} & \left(\Delta^{\mathrm{DT}}\left[s_{0}, \gamma_{n}\right] \in \complement \Theta_{n}^{(4)}\right) \\
& =\mathrm{P}_{n f\left(s_{0}\right)}\left(\left.\sup _{l \in \llbracket 1, \gamma_{n} \rrbracket}\left|\sum_{j=1}^{l} n^{(g-1) / 2}\right| \Delta^{\mathrm{DT}}\left(s_{0}, j\right)\right|^{g}-\frac{\boldsymbol{C}_{\text {bis }}^{T, g}}{\sqrt{n} f\left(s_{0}\right)^{g / 2}} \mid \geq y_{n}\right) \\
& \leq \mathrm{P}_{1}\left(\sup _{l \in \llbracket 1, \gamma_{n} \rrbracket}\left|\sum_{j=1}^{l} \frac{\left|\Delta^{\mathrm{DT}}\left(s_{0}, j\right)\right|^{g}-C_{\text {bis }}^{T, g}}{\sqrt{\gamma_{n}}}\right| \geq y_{n} f\left(s_{0}\right)^{g / 2} \frac{\sqrt{n}}{\sqrt{\gamma_{n}}}\right) .
\end{aligned}
$$

Letting $y_{n}=x_{\gamma_{n}} \sqrt{n} / \sqrt{\gamma_{n}} m_{f}^{g / 2}$ for $x_{n} \sim n^{w^{\prime}}$, we complete the proof in the same way as Theorem 5. Note that the deviations of $\boldsymbol{C}^{(n)}$ are of the same order as those of $\boldsymbol{Z}^{(n)}$ in the proof of Theorem 5. Now, $\Delta^{\mathrm{DT}} \in \Theta_{n}^{(1)} \cap \Theta_{n}^{(4)}$ with a probability exponentially close to 1 , and, therefore, the conclusion of the theorem holds for the pair $\left(\boldsymbol{Z}^{(n)}, \boldsymbol{C}^{(n)}\right)$.

Limits in the straight case. Here $\boldsymbol{X} \in\{\mathrm{ST}, \mathrm{SY}\}$. Consider $\left(\mathrm{Sol}_{s}^{C_{\text {bis }}^{X}}, \arg t-s, C^{\boldsymbol{X}, g}\right)$, the solution of $\operatorname{ODE}^{(2)}\left(\boldsymbol{C}_{\mathrm{bis}}^{\boldsymbol{X}}, \arg (t-s), \boldsymbol{C}_{\mathrm{bis}}^{\boldsymbol{X}, g}, s, 0\right)$, and consider $\operatorname{Time}_{s, t} \boldsymbol{C}_{\text {bis }}^{\boldsymbol{X}}$. The limiting cost will be $C_{s, t}^{X, g}:=C^{\boldsymbol{X}, g}\left(\right.$ Time $\left._{s, t}^{\boldsymbol{C}_{\text {bis }}^{\boldsymbol{X}}}\right)$. Note that if $g=1$, then $C^{\boldsymbol{X}, 1}(x)=\mathrm{E}\left(\left|\Delta_{1}^{\boldsymbol{X}}\right|\right)\left|\mathrm{Pos}_{s, t}^{\boldsymbol{C}_{\text {bis }}^{X}}-s\right|$.

Theorem 8. Let $\boldsymbol{X}=\mathrm{SY}$ and $\theta<\pi / 2$, or $\boldsymbol{X}=\mathrm{ST}$ and $\theta \leq \pi / 2$. For any $\alpha \in\left(0, \frac{1}{8}\right)$, any $\beta>0$, any $\lambda>0$, and large enough $n$,

$$
\mathrm{P}_{n f}\left(\sup _{(s, t) \in D^{\prime}[a]}\left|\frac{\operatorname{Cost}_{H_{g}}^{X}(s, t)}{n^{1 / 2-g / 2}}-C_{s, t}^{X, g}\right| \geq n^{-\alpha}\right) \leq n^{-\beta} .
$$


The proof follows that of Theorem 6: first a proof for fixed $(s, t)$ is obtained, then the proof is extended to a subgrid of $\mathscr{D}[a]^{2}$ and then to all pairs using the arguments of Section 4.3.

The proof for fixed $(s, t)$ is similar to that of Theorem 6: the contribution to the cost of the stages of the traveller outside the final ball $B\left(t, n^{-\alpha}\right)$ is provided by the solution of a differential equation. Then, when the traveller enters the final balls $B\left(t, n^{-\alpha}\right)$ and $B\left(t, n^{B-1 / 2}\right)$, we again use the fact that these final contributions are negligible, affecting the total cost by a negligible amount (smaller than $n^{-\alpha}$ with a large probability).

Limits in the cross case. Here $X \in\{\mathrm{CT}, \mathrm{CY}\}$. Again, we must use the decomposition of the limiting path at the point $I(s, t)$. From $s$ to $I(s, t)$, denote by $\left(\operatorname{Sol}_{s} C_{\text {bis }}^{X}, \arg I(s, t)-s, C_{1}^{X, g}\right)$ the solution of $\operatorname{ODE}^{(2)}\left(\boldsymbol{C}_{\mathrm{bis}}^{\boldsymbol{X}}, \arg (I(s, t)-s), \boldsymbol{C}_{\mathrm{bis}}^{X, g}, s_{0}, 0\right)$ on the time interval $\left[0, \operatorname{Time}_{s, I(s, t)}^{\boldsymbol{C}_{\mathrm{bis}}^{X}}\right]$; between $I(s, t)$ and $t$, let $\left(\operatorname{Sol}_{I(s, t)}^{\boldsymbol{C}_{\text {bor }}^{\boldsymbol{X}}, \arg t-I(s, t)}, C_{2}^{\boldsymbol{X}, g}\right)$ be the solution of $\operatorname{ODE}^{(2)}\left(\boldsymbol{C}_{\mathrm{bor}}^{\boldsymbol{X}}, \arg (t-\right.$ $\left.I(s, t)), C_{\mathrm{bis}}^{X, g}, I(s, t), 0\right)$ on the time interval $\left[0, \operatorname{Time}_{I(s, t), y}^{C_{\mathrm{bor}}^{X}}\right]$. In this case the limiting cost will be

$$
\boldsymbol{C}_{s, t}^{\boldsymbol{X}, g}:=C_{1}^{\boldsymbol{X}, g}\left(\text { Time }_{s, I(s, t)}^{\boldsymbol{C}_{\text {bis }}^{\boldsymbol{X}}}\right)+C_{2}^{\boldsymbol{X}, g}\left(\text { Time }_{I(s, t), t}^{\boldsymbol{C}_{\mathrm{bor}}^{\boldsymbol{X}}}\right) .
$$

Theorem 9. For any $\theta \leq \pi / 3$, any $\boldsymbol{X} \in\{\mathrm{CT}, \mathrm{CY}\}$, any $\alpha \in\left(0, \frac{1}{8}\right)$, any $\beta>0$, any $\lambda>0$, and large enough $n$,

$$
\mathrm{P}_{n f}\left(\sup _{(s, t) \in \mathscr{D}^{\prime}[a]}\left|\frac{\operatorname{Cost}_{H_{g}}^{\boldsymbol{X}}(s, t)}{n^{1 / 2-g / 2}}-\boldsymbol{C}_{s, t}^{\boldsymbol{X}, g}\right| \geq n^{-\alpha}\right) \leq n^{-\beta} .
$$

\subsection{Globalisation of the bounds}

We have obtained some bounds for the position and the cost functions of a traveller going from $s$ to $t$. Here we want to prove some uniform bounds since in the main theorems a supremum on $(s, t)$ lies inside the considered probabilities. With the number of possible pairs $(s, t)$ being infinite, the union bound is not sufficient here. We adopt a two-point strategy to obtain the uniformity needed. First we obtain the uniformity for pairs $(s, t)$, where $s$ and $t$ belong to a subgrid of $\mathcal{D}[a]$ :

$$
\operatorname{Grid}_{n}\left(c_{0}, a\right):=n^{-c_{0}} \mathbb{Z}^{2} \cap \mathcal{D}[a] .
$$

With the cardinality of $\operatorname{Grid}_{n}\left(c_{0}, a\right)$ being $O\left(n^{2 c_{0}}\right)$, by the union bound, any theorem of the form

$$
\mathrm{P}_{n f}\left(h\left(\operatorname{Path}_{s, t}^{X}\right) \in A\right)=O\left(u_{n}\right) \quad \text { for all }(s, t) \in \mathscr{D}^{\prime}[a],
$$

where $u_{n}$ does not depend on $s$ and $t$, and $h$ is any function of the paths, has the corollary

$$
\mathrm{P}_{n f}\left(\sup _{(s, t) \in \operatorname{Grid}_{n}\left(c_{0}, a\right)} h\left(\operatorname{Path}_{s, t}^{X}\right) \in A\right)=O\left(n^{2 c_{0}} u_{n}\right) .
$$

In other words, if the probability concerning one path is exponentially small (this is the case for most of our theorems concerning one trajectory) then it is still exponentially small when considering all paths starting and ending in $\operatorname{Grid}_{n}\left(c_{0}, a\right)$.

The second point of our strategy is the following. Take any $(s, t) \in \mathscr{D}^{\prime}[a]$, say, in the complement of $\operatorname{Grid}_{n}\left(c_{0}, a\right)$. We will show that, with a probability close to 1 , the trajectory from $s$ to $t$ can be split with a large probability into at most 13 parts (uniformly on $(s, t)$ ), such that on each part the path of the traveller coincides with a part of the path of a traveller starting and ending on the grid. This will be sufficient to conclude, since our theorems control the behaviour of the whole path of a traveller, and, therefore, also along the aforementioned parts. 
To this end, we introduce $\operatorname{Squ}_{n}^{*}\left(c_{0}\right)$, the set of squares in the plane with side length $n^{-c_{0}}$ and vertices in $n^{-c_{0}} \mathbb{Z}^{2}$, with at least one vertex in $\operatorname{Grid}_{n}\left(c_{0}, a\right)$.

Additionally, consider four tilings of the plane with squares of length

$$
a_{n}:=n^{B-1 / 2}
$$

the three last tilings are obtained from the first tiling by the translations $a_{n} / 2, \mathrm{i} a_{n} / 2$, and

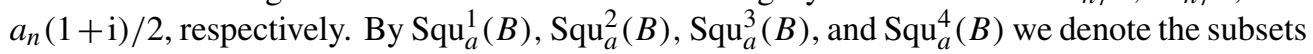
of the squares of each of these tilings having a distance to $\mathscr{D}[a]$ smaller than $a_{n}$, and by $\operatorname{Squ}_{a}(B)$ we denote their union. If $n$ is large enough, the union of the squares of $\operatorname{Squ}_{a}(B)$ contains $\mathscr{D}[a]$ and are included in the interior of $\mathscr{D}$ (for example, in $\mathscr{D}[a / 2]$ ). Observe also that any disk $B\left(x, a_{n} / 4\right)$ with $x \in \mathscr{D}[a]$ is totally included in a square of $\operatorname{Squ}_{a}(B)$.

With any $(s, t) \in \mathscr{D}^{\prime}[a]$ we will associate two points $\left(s_{g}, t_{g}\right)$ belonging to $\operatorname{Grid}_{n}\left(c_{0}, a\right)$ as follows. First $t_{g}$ is the upper-left corner of the square of $\operatorname{Squ}_{n}^{*}\left(c_{0}\right)$ containing $t$ (therefore, $t_{g}$ depends on $n$ and $\left.c_{0}\right)$; if $t$ is in $\operatorname{Squ}^{\star}\left(c_{0}\right)$ then take $t_{g}=t$. The point $s_{g}$ is given by the following lemma.

Lemma 10. Let $s \in \mathscr{D}$, and consider one of the navigation processes presented in this paper using as a set of stopping places $S$. For any $\rho<r_{\min }(S):=\min \{|x-y|, x, y \in S, x \neq y\}$, and, for any pair $\left(s^{\prime}, t\right)$ of $S \times \mathscr{D}$, there exists a point $s_{g}$ in $\rho \mathbb{Z}^{2} \cap \mathbb{D}$ such that $\boldsymbol{X}\left(s_{g}, t\right)=\boldsymbol{X}(s, t)=s^{\prime}$, i.e. the paths from $s$ and $s_{g}$ to $t$ coincide from the second stage.

Proof. First observe that around $s^{\prime}$ there is a disk of radius $r_{\min }(S)$ containing no other vertex of $S$. For a straight navigation process, let us define $R=\overrightarrow{\mathrm{Cam}}\left(s^{\prime}, s^{\prime}-t\right)\left(r_{\min }(S) / 2\right)$, and, for a cross navigation process, let us define $R=\operatorname{Cam}[s,-k]\left(r_{\min }(S) / 2\right)$, where $k$ is such that $t \in \operatorname{Sect}\left[s^{\prime}, k\right]$. By construction, any point $s$ of $R$ is such that $\boldsymbol{X}(s, t)=s^{\prime}$. Here $R$ contains a disk of radius

$$
r=\frac{r_{\min }(S)}{2} \frac{\sin (\theta / 2)}{1+\sin (\theta / 2)}>\frac{\theta}{4 \pi}
$$

(see Figure 10). This disk intersects at least one point of $\rho \mathbb{Z}^{2} \cap \mathscr{D}$.

Lemma 11. For any $c>0, \mathrm{P}_{n f}\left(r_{\min } \leq n^{-c}\right)=O\left(n^{-2 c+2}\right)$.

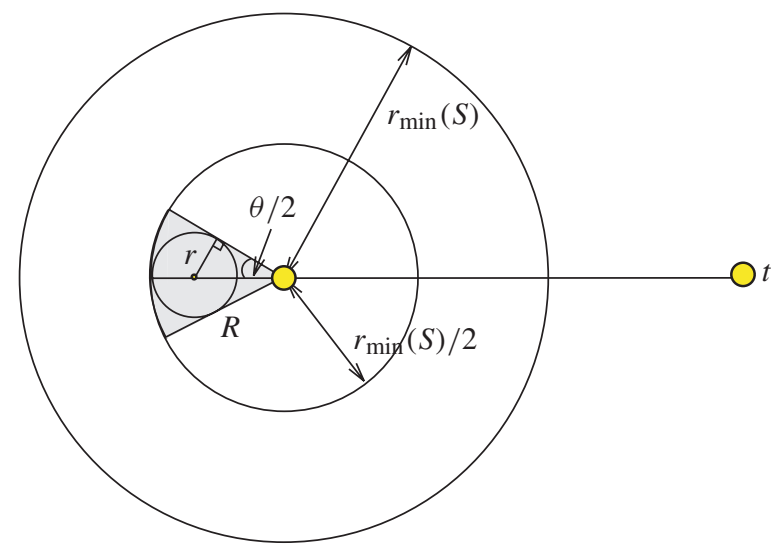

FIGURE 10: An illustration of the proof of Lemma 10. 
Proof. With a $\mathrm{P}_{n f}$ probability exponentially close to $1,|\# \boldsymbol{S}|$ is smaller than $c_{1} n$ for some $c_{1}>0$ (see the proof of Lemma 3). By the union bound,

$$
\mathrm{P}_{n f}\left(r_{\min } \leq \lambda|| \# \boldsymbol{S} \mid=m\right) \leq m^{2} \mathrm{P}_{n f}\left(\left|V_{1}-V_{2}\right| \leq \lambda\right)
$$

for $V_{1}$ and $V_{2}$ independent with density $f$ on $\mathscr{D}$. Conditioning on $V_{1}$, it is easily seen that this probability is smaller than $c_{2} \lambda^{2}$ (for some $c_{2}$ independent on $\lambda$ ). Finally, we obtain $\mathrm{P}_{n f}\left(r_{\min } \leq\right.$ $\lambda) \leq c_{1}^{2} n^{2} c_{2} \lambda^{2}+\mathrm{P}_{n f}\left(|\# \boldsymbol{S}| \geq c_{1} n\right)$, which leads easily to the stated result.

A consequence of the two previous lemmas is that if $c_{0}$ is large enough, with a probability $O\left(n^{-2 c_{0}+2}\right)$, any path from $s$ to $t$ coincides with a path from $s_{g}$ to $t$ for a point $s_{g}$ in $\operatorname{Grid}_{n}\left(c_{0}, a\right)$ up to the starting position (and then, up to the first step, smaller than Navmax, which will then be uniformly negligible at the scale we are working in). Hence, to approximate a path from $s$ to $t$ with a path from $s_{g}$ to $t_{g}$, most of the difficulties come from the target.

Given a source $s$, a section of the Path $(s, t)=\left(s_{0}, \ldots, s_{\mathrm{Nb}(s, t)}\right)$ is a subpath $\left(s_{j_{1}}, \ldots, s_{j_{2}}\right)$ for which $\boldsymbol{X}(s, t, j)=\boldsymbol{X}\left(s_{j-1}, t_{g}\right)$ for each $1 \leq j_{1}<j<j_{2}$, that is, a part of $\operatorname{Path}(s, t)$ matching a path toward $t_{g}$. Given a source $s$ and a target $t$, a $\operatorname{Squ}_{a}(B)$-black box is a subpath $\left(s_{j_{1}}, \ldots, s_{j_{2}}\right)$ of $\operatorname{Path}(s, t)$ such that $s_{j_{1}}$ and $s_{j_{2}}$ are both in the same square of $\operatorname{Squ}_{a}(B)$. For $k \in \mathbb{N}$, we will say that a path admits a $\left(k, B, c_{0}\right)$ decomposition if it can be decomposed into at most $k$ sections and $k \operatorname{Squ}_{a}(B)$ black boxes.

Proposition 5. Let $B>0$ and $\rho>0$ be fixed. For any $\boldsymbol{X} \in\{\mathrm{CY}, \mathrm{CT}\}$ and $\theta \leq \pi / 3$, or $\boldsymbol{X}=\mathrm{ST}$ and $\theta \leq \pi / 2$, or $\boldsymbol{X}=\mathrm{SY}$ and $\theta<\pi / 2$, there exists $c_{0}>0$ such that

$$
\mathrm{P}_{n f}\left(\text { for all }(s, t) \in \mathcal{D}^{\prime}[a], \operatorname{Path}^{X}(s, t) \text { admits a }\left(13, B, c_{0}\right) \text { decomposition }\right) \geq 1-o\left(n^{-\rho}\right) \text {. }
$$

Before proving this proposition, let us examine how it entails, together with the already proved results concerning one trajectory, the theorems of this paper.

\subsection{Consequences}

From the above discussion, the results concerning one trajectory, Theorems 6 and 7 , can be extended as follows: a supremum on $(s, t)$ in $\operatorname{Grid}\left(c_{0}, a\right)$ can be added inside the probabilities present in these theorems. It remains to see what is the price of taking the supremum on all $(s, t)$. We saw above that, with a probability polynomially close to 1 , for any $(s, t), \operatorname{Path}(s, t)$ can be decomposed into at most 13 sections and 13 black boxes. On each section, $\operatorname{Path}(s, t)$ coincides with a section of $\operatorname{Path}\left(s, t_{g}\right)$. Therefore, the fluctuations of the position functions (or the cost functions) on each of these sections are smaller than $n^{-\alpha}$ with a probability exponentially close to 1 , since this is the case for the trajectories between the points of $\operatorname{Grid}_{n}\left(c_{0}, a\right)$. To see this, assume for example that

$$
\sup _{x \in[0, \lambda]}\left|\operatorname{Pos}_{s, t}^{X}(x \sqrt{n})-\operatorname{Pos}_{s, t}^{\infty, C_{\text {bis }}^{X}}(x)\right| \leq \varepsilon .
$$

Therefore, for any increasing sequence $x_{1}, \ldots, x_{2 k}$ (where $x_{2 i-1} \sqrt{n}$ and $x_{2 i} \sqrt{n}$ have to be understood as the starting and ending times of the sections), we have

$$
\begin{aligned}
\sup _{0 \leq x_{1} \leq \cdots \leq x_{2 k} \leq \lambda} & \left|\sum_{i=1}^{k}\right| \operatorname{Pos}_{s, t}^{X}\left(x_{2 i} \sqrt{n}\right)-\operatorname{Pos}_{s, t}^{X}\left(x_{2 i-1} \sqrt{n}\right) \mid \\
& -\sum_{i=1}^{k}\left|\operatorname{Pos}_{s, t}^{\infty, C_{\text {bis }}^{X}}\left(x_{2 i}\right)-\operatorname{Pos}_{s, t}^{\infty, C_{\text {bis }}^{X}}\left(x_{2 i-1}\right)\right| \mid \leq k \varepsilon .
\end{aligned}
$$




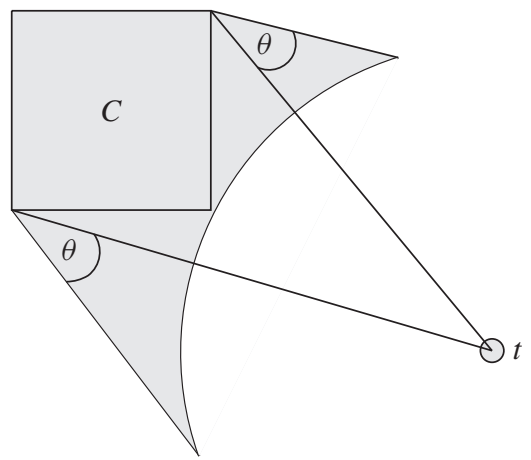

FIGURE 11: The area around $\square$ containing the range of the black boxes $\left(s_{j_{1}}, \ldots, s_{j_{2}}\right)$.

Hence, a global control of the path ensures a good control of the sections, provided that the number of sections is small. This is the case here, since we have 13 sections at most with a large probability. Now, the contribution of the black boxes (the stages between times $x_{2 i} \sqrt{n}$ and $x_{2 i+1} \sqrt{n}$ ) have to be controlled. For this, note first that the space fluctuations for any black box $\left(s_{j_{1}}, \ldots, s_{j_{2}}\right)$ is small: $\max \left\{\left|s_{j}-s_{j_{1}}\right|, j \in \llbracket j_{1}, j_{2} \rrbracket\right\} \leq c a_{n}$ for a constant $c$ depending only on $\theta$; indeed, since $s_{j_{1}}$ and $s_{j_{2}}$ are in the same square, $\square$, of $\operatorname{Squ}_{a}(B)$, and since at each stage the traveller gets closer to the target, and since the angle between its local direction and the direction to the target is smaller than $\theta$ (see Figure 11), it must stay in a domain with area at most $O\left(a_{n}^{2}\right)$ (which is included in the ball $B\left(\circ, c a_{n}\right)$, where $\circ$ is the centre of $\square$ ). Moreover, let $l(\boldsymbol{X})$ be the maximum length (number of stages) of all black boxes for the algorithm $\boldsymbol{X} \in\{\mathrm{CT}, \mathrm{CY}, \mathrm{ST}, \mathrm{SY}\}$ :

$$
l(\boldsymbol{X}):=\max \left\{j_{2}-j_{1},\left(s_{j_{1}}, \ldots, s_{j_{2}}\right) \text { black box }\right\} .
$$

Then $l(\boldsymbol{X})$ bounded by $\operatorname{Maxball}\left(c a_{n}, a\right)$, and

$$
\mathrm{P}\left(l(\boldsymbol{X}) \geq a_{n}^{2} n^{1+\varepsilon}\right)=\mathrm{P}\left(l(\boldsymbol{X}) \geq n^{2 B+\varepsilon}\right) \leq \exp \left(-n^{d}\right)
$$

for some $d>0$ if $n$ is large enough. Since $2 B+\varepsilon$ can be chosen smaller than $\frac{1}{2}$ in all the proofs, we have at most $k$ black boxes, of negligible size $n^{2 B+\varepsilon}$. The contributions of these black boxes to the time normalisation of the processes are negligible, as well as the space normalisation: at most $a_{n}^{2} n^{1+\varepsilon}$ contributions of size $n^{C}$ with size smaller than Navmax or $H(\operatorname{Navmax} / \sqrt{n})$ are (uniformly for all $(s, t)$ ) negligible.

\subsection{Proof of Proposition 5}

In order to bound the number of black boxes on a trajectory, we need to understand when a navigation decision differs when navigating to $t$ or $t_{g}$. We will see that this is related to some geometrical features of some straight lines, called 'rays' below, issued from the points of $S$.

A star is a collection of half-lines (we also use the term rays) issued from the same point called the centre. A navigation star of $s \in S$ is a cyclic ordered list $\left(r_{1}, r_{2}, \ldots, r_{k}\right)$ of half-lines starting at $s$ such that, for any two points $t$ and $t^{\prime}$ of $\mathscr{D}$ between two consecutive half-lines, if $\boldsymbol{X}(s, t) \neq t$ and $\boldsymbol{X}\left(s, t^{\prime}\right) \neq t^{\prime}$ then $\boldsymbol{X}(s, t)=\boldsymbol{X}\left(s, t^{\prime}\right)$; in other words, if $t$ and $t^{\prime}$ are far enough from $s$ (at a distance of at least 2 Navmax) then the first stop starting from $s$ is the same whether the target point is $t$ or $t^{\prime}$ (see Figure 12). 

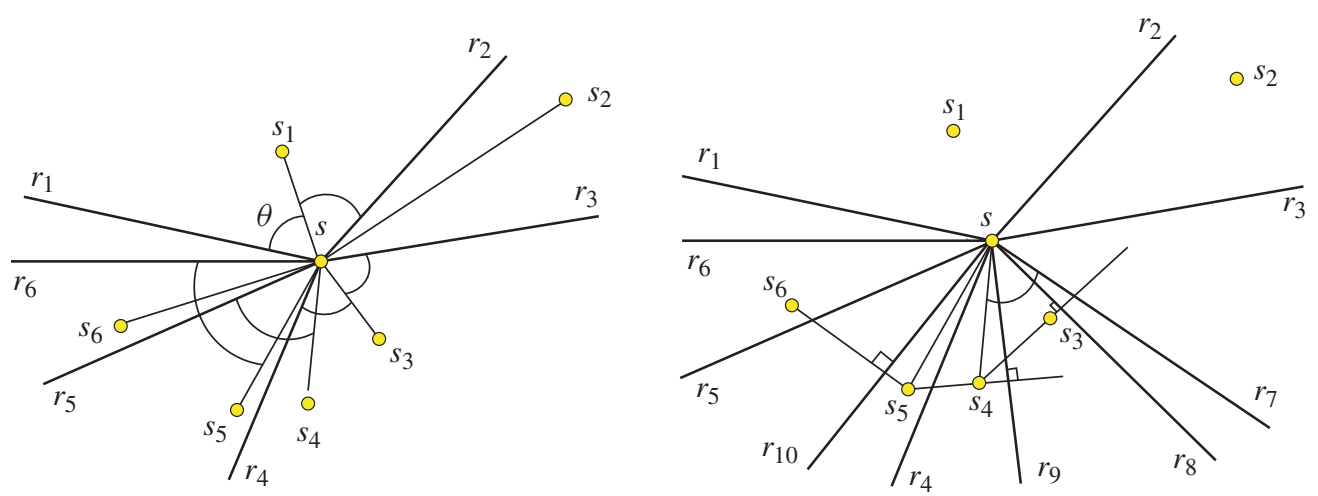

FiguRE 12: Left: an example of a navigation star in $\theta$-straight Yao navigation. For a vertex $t$ far enough from $s$ between $r_{i}$ and $r_{i+1}, \mathrm{SY}(s, t)=s_{i}$ (assuming that $r_{7}=r_{1}$ ). Right: an example of a navigation star in $\theta$-straight $\mathrm{T}$ navigation. In this case the situation is a bit more complex. For instance, $\operatorname{ST}(s, t)=s_{3}$ if $t$ is between $r_{3}$ and $r_{7}$ or between $r_{8}$ and $r_{4}$. If $t$ is between $r_{7}$ and $r_{8}$ (and far enough from $s$ ) then $\mathrm{ST}(s, t)=s_{4}$.

In order to build navigation stars, we define different types of ray; they will be used to control the decompositions of straight or cross navigation paths in the sequel.

- Given a point $s$ of $S$ and $k \in \llbracket 1, p_{\theta} \rrbracket$, the $k$ th type- 1 ray around $s$ is simply $r_{1}(s, k)=$ $\mathrm{HL}_{k}(s)$.

- Let $s$ be a point of $S$. We construct two rays associated with each points $s^{\prime}$ of $S$. Denote by $r_{2}^{+}\left(s, s^{\prime}\right)$ and $r_{2}^{-}\left(s, s^{\prime}\right)$ the half-lines with centers $s$ obtained from the half-line $\left[s, s^{\prime}\right)$ by rotations of $\theta / 2$ and $-\theta / 2$, respectively. These rays are called type- 2 rays.

- Let $s$ be a point of $S$. For any pair $\left(s^{\prime}, s^{\prime \prime}\right)$ of $S^{2}$, we denote by $r_{3}\left(s, s^{\prime}\right)$ the half-line $[s, t)$ such that $[s, t)$ is orthogonal to $\left(s^{\prime}, s^{\prime \prime}\right)$. This ray is called a type-3 ray.

We now examine the navigation stars of the different algorithms. It may help to note that if NS is a navigation star around $s$ then any set of rays containing NS is also a navigation star.

Cross navigations. For cross navigations, the set of type- 1 rays centred at $s$, i.e. $\operatorname{Cross}(s)$, is a navigation star of $s$.

$\theta$-straight Yao navigation. Starting from $s$ for a target $t$ far enough from $s, \mathrm{SY}(s, t)$ depends only on $\arg (t-s)$. When $t$ is turning around $s, \mathrm{SY}(s, t)$ changes when the nearest point to $s$ in $\overrightarrow{\operatorname{Sect}}(s, t) \cap S$ changes. This potentially happens when the angle between $(s, t)$ and $\left(s, s^{\prime}\right)$ is $\pm \theta / 2$ for a point $s^{\prime}$ in $S$ close enough to $s$, i.e. $s^{\prime}$ is such that $\left|s-s^{\prime}\right| \leq$ Navmax. Hence, the set of type- 2 rays centred at $s$ is a navigation star of $s$ for $\theta$ straight Yao navigation.

$\theta$-straight T navigation. In addition to the rays presented in the previous point, we need to consider another type. Note that $\mathrm{ST}(s, t)$ is the point of $\overrightarrow{\operatorname{Sect}}(s, t) \cap S$ that has the closest to $s$ orthogonal projection on the bisecting line of $\overrightarrow{\operatorname{Sect}}(s, t) \cap S$. When $t$ is turning around $s, \mathrm{ST}(s, t)$ potentially changes when the order of the orthogonal projections of the elements of $\overrightarrow{\operatorname{Sect}}(s, t) \cap S$ on the bisecting line of $\overrightarrow{\operatorname{Sect}}(s, t)$ changes. This may happen when the line that passes via two elements $s^{\prime}$ and $s^{\prime \prime}$ of $S$, not too far from $s$ (such 
that $\left|s-s^{\prime}\right| \leq$ Navmax and $\left|s-s^{\prime \prime}\right| \leq$ Navmax), is orthogonal to the line $(s, t)$. Hence, the set of type- 2 rays and type- 3 rays centred at $s$ is a navigation star of $s$ for $\theta$-straight T navigation.

A navigation star containing only the rays defined in the previous paragraph is called standard. A constellation or standard constellation of $S$ is the union of the navigation stars or standard navigation stars, respectively, for every $s \in S$.

Given $C_{t} \in \operatorname{Squ}_{n}^{*}\left(c_{0}\right)$ and $C \in \operatorname{Squ}_{a}(B)$, denote by $\operatorname{Numb}_{i}\left(C, C_{t}\right)$ the number of type- $i$ rays that intersect $C$ and are defined by points $s, s^{\prime}$, and $s^{\prime \prime}$ in $S \cap C$ (depending on the case, $s^{\prime}$ and $s^{\prime \prime}$ can be non-necessary). Also, define

$$
\begin{aligned}
& \operatorname{Numb}^{\mathrm{CT}}\left(C, C_{t}\right)=\operatorname{Numb}^{\mathrm{CY}}\left(C, C_{t}\right)=\operatorname{Numb}_{1}\left(C, C_{t}\right), \\
& \operatorname{Numb}^{\mathrm{SY}}\left(C, C_{t}\right)=\operatorname{Numb}_{2}\left(C, C_{t}\right), \\
& \operatorname{Numb}^{\mathrm{ST}}\left(C, C_{t}\right)=\operatorname{Numb}_{2}\left(C, C_{t}\right)+\operatorname{Numb}_{3}\left(C, C_{t}\right),
\end{aligned}
$$

as is clear from the above discussion. We also set $\operatorname{Numb}\left(C, C_{t}\right)=\sum_{i=1}^{3} \operatorname{Numb}_{i}\left(C, C_{t}\right)$, a bound on all the $\operatorname{Numb}^{X}\left(C, C_{t}\right)$ for $\boldsymbol{X} \in\{\mathrm{ST}, \mathrm{SY}, \mathrm{CT}, \mathrm{CY}\}$.

Lemma 12. Let $B>0$ and $c_{0}>0$ be fixed. For any algorithm $\boldsymbol{X} \in\{\mathrm{ST}, \mathrm{SY}, \mathrm{CT}, \mathrm{CY}\}$,

$$
\sup _{\substack{\left(C, C_{t}\right) \in \operatorname{Squ}_{a}(B) \times \operatorname{Squ}_{n}^{*}\left(c_{0}\right) \\ d\left(C, C_{t}\right) \geq a_{n}}} \mathrm{P}_{n f}\left(\mathrm{Numb}^{X}\left(C, C_{t}\right) \geq 1\right)=O\left(n^{4 B+2-c_{0}}\right) .
$$

Proof. Throughout the proof, $C_{t} \in \operatorname{Squ}_{n}^{*}\left(c_{0}\right)$ and $C \in \operatorname{Squ}_{a}(B)$ are such that $d\left(C_{t}, C\right) \geq a_{n}$. We first control $\operatorname{Numb}_{1}\left(C, C_{t}\right)$. Let $\operatorname{St}\left(C_{t}\right)$ be the set of points $s \in \mathscr{D}[a]$ such that $\operatorname{Cross}(s)$ crosses $C_{t}$. This set also forms a cross. Clearly, for any $i \in\{1,2,3\}$,

$$
\mathrm{P}_{n f}\left(\mathrm{Numb}_{i}\left(C, C_{t}\right) \geq 1\right) \leq \mathrm{P}_{n M_{f}}\left(\operatorname{Numb}_{i}\left(C, C_{t}\right) \geq 1\right) .
$$

We then obtain the bound under $\mathrm{P}_{n M_{f}}$ instead than $\mathrm{P}_{n f}$. Now,

$$
\mathrm{P}_{n M_{f}}\left(\operatorname{Numb}_{1}\left(C, C_{t}\right) \geq 1\right) \leq \mathrm{P}_{n M_{f}}\left(\#\left(S \cap C \cap S t\left(C_{t}\right)\right) \geq 1\right)=O\left(n\left|C \cap S t\left(C_{t}\right)\right|\right),
$$

where $|A|$ denotes the area of $A$ (since, for an integer random variable $X, \mathrm{P}(X \geq 1) \leq \mathrm{E}(X)$ ). Observing that $S t\left(C_{t}\right)$ is composed of $p_{\theta}$ stripes having width $O\left(n^{-c_{0}}\right)$ and that $C$ has diameter $\sqrt{2} n^{B-1 / 2}$, we have $\left|C \cap S t\left(C_{t}\right)\right|=O\left(n^{B-1 / 2-c_{0}}\right)$, and then

$$
\mathrm{P}_{n f}\left(\mathrm{Numb}_{1}\left(C, C_{t}\right) \geq 1\right)=O\left(n^{1 / 2+B-c_{0}}\right) .
$$

We now discuss the control of Numb 2 . For $p$ the centre of $C_{t}$, we have

$$
\begin{aligned}
& \mathrm{P}_{n f}\left(\operatorname{Numb}_{2}\left(C, C_{t}\right) \geq 1\right) \\
& \quad \leq \mathrm{P}_{n M_{f}}\left(\text { there exist } X_{1}, X_{2} \in S \cap C,|| \arg \left(X_{2}-X_{1}\right)-\arg \left(p-X_{1}\right)\left|-\frac{\theta}{2}\right|\right. \\
& \left.\quad \leq \arctan \left(\frac{n^{-c_{0}}}{\sqrt{2}\left|p-X_{1}\right|}\right)\right) .
\end{aligned}
$$

Since $\left|X_{1}-p\right|>n^{B-1 / 2}, \arctan \left(n^{-c_{0}} / \sqrt{2}\left|p-X_{1}\right|\right) \leq \arctan \left(n^{-c_{0}-B+1 / 2} / \sqrt{2}\right)$. Moreover, using (13), $\mathrm{P}_{n M_{f}}\left(|S \cap C|>n^{2 B+d}\right) \leq \exp \left(-n^{2 B+\bar{d}}\right)$ for any $d>0$, provided that $n$ is 
large enough. Recall now that, under $\mathrm{P}_{n M_{f}}$, knowing that $\#(S \cap C)=k$, the set $S \cap C$ is distributed as a sample of $k$ i.i.d. uniform random variables in $C$. Therefore, for any function $g$ and measurable set $A$,

$$
\begin{aligned}
& \left.\mathrm{P}_{n M_{f}} \text { (there exist } X_{1}, X_{2} \in S, X_{1} \neq X_{2}, g\left(X_{1}, X_{2}\right) \in A\right) \\
& \leq \mathrm{P}_{n M_{f}}\left(\#\left(S \cap C>n^{2 B+d}\right)\right) \\
& \left.\quad+\sum_{k \leq n^{2 b+d}} \mathrm{P}_{n M_{f}} \text { (there exist } X_{1}, X_{2} \in \boldsymbol{S} \cap C, g\left(X_{1}, X_{2}\right) \in A \mid \# \boldsymbol{S}=k\right) \\
& \quad \times \mathrm{P}_{n M_{f}}(\# \boldsymbol{S}=k) \\
& \leq \mathrm{P}_{n M_{f}}\left(\#\left(S \cap C>n^{2 B+d}\right)\right)+\left(n^{2 B+d}\right)^{2} \mathrm{P}\left(g\left(U_{1}, U_{2}\right) \in A\right)
\end{aligned}
$$

for two i.i.d. uniform random variables $U_{1}$ and $U_{2}$ in $C$. Therefore, $\mathrm{P}_{n M_{f}}\left(\operatorname{Numb}_{2}\left(C, C_{t}\right) \geq 1\right)$ is bounded by

$$
\begin{aligned}
& O\left(\left(n^{2 B+d}\right)^{2}\right) \mathrm{P}\left(|| \arg \left(U_{2}-U_{1}\right)-\arg \left(p-U_{1}\right)\left|-\frac{\theta}{2}\right|\right. \\
& \left.\quad \leq \arctan \left(\frac{n^{-c_{0}}}{\sqrt{2}\left|p-U_{1}\right|}\right)\right)+\exp \left(-n^{2 B+d}\right) .
\end{aligned}
$$

A simple picture shows that, $U_{1}$ given, $U_{2}$ must lie in a triangle included in $C$, with diameter smaller than $\sqrt{2} a_{n}$ and with an angle smaller than $O\left(n^{-c_{0}-B+1 / 2} / \sqrt{2}\right)$. Since the density of $U_{2}$ is $1 / a_{n}^{2}$, for any $U_{1}$ given in $C$, the probability is $O\left(n^{-c_{0}-B+1 / 2} a_{n} / a_{n}^{2}\right)=O\left(n^{-c_{0}-2 B+1}\right)$ (with a constant in the $O$ sign uniform for $U_{1}$ in $C$ ). Then

$$
\mathrm{P}_{n f}\left(\mathrm{Numb}_{2}\left(C, C_{t}\right) \geq 1\right)=O\left(n^{4 B+2 d}\right) O\left(n^{-c_{0}-2 B+1}\right)+\exp \left(-n^{2 B+d}\right)=O\left(n^{2 B+2 d-c_{0}+1}\right) .
$$

Taking small enough $d$, we obtain $\mathrm{P}_{n f}\left(\operatorname{Numb}_{2}\left(C, C_{t}\right) \geq 1\right)=O\left(n^{2 B-c_{0}+2}\right)$.

We now discuss the control of $\mathrm{Numb}_{3}$. The quantity $\mathrm{P}_{n f}\left(\mathrm{Numb}_{3}\left(C, C_{t}\right) \geq 1\right)$ is bounded by

$$
\begin{aligned}
& \mathrm{P}_{n M_{f}}\left(\text { there exist } X_{1}, X_{2}, X_{3} \in S \cap C,\left|\arg \left(X_{3}-X_{2}\right)-\arg \left(p-X_{1}\right)-\frac{\pi}{2}\right|\right. \\
& \left.\quad \leq \arctan \left(\frac{n^{-c_{0}-B+1 / 2}}{\sqrt{2}}\right)\right) .
\end{aligned}
$$

Again, this is

$$
O\left(\left(n^{2 B+d}\right)^{3}\right) \mathrm{P}\left(\left|\arg \left(U_{3}-U_{2}\right)-\arg \left(p-U_{1}\right)-\frac{\pi}{2}\right| \leq \arctan \left(n^{-c_{0}-B+1 / 2}\right)\right)
$$

for some $U_{1}, U_{2}$, and $U_{3}$ i.i.d. uniform in $C$. For $\left(U_{1}, U_{2}\right)$ given, $U_{3}$ must be in a subset of $C$ with Lebesgue measure $O\left(n^{-c_{0}-B+1 / 2} a_{n}\right)$. Taking into account the density of $U_{3}$, we obtain

$$
\mathrm{P}_{n f}\left(\mathrm{Numb}_{3}\left(C, C_{t}\right) \geq 1\right)=O\left(n^{4 B-c_{0}+2}\right)
$$

for $d$ chosen small enough.

We conclude this proof using (28), (29), (30), and the union bound. 
For any $C_{t} \in \mathrm{Squ}_{c_{0}}^{*}$, let $\mathrm{BB}\left(C_{t}\right)=\left\{C \in \mathrm{Squ}_{a}(B), \operatorname{Numb}\left(C, C_{t}\right) \geq 1\right\}$ be the number of squares in $\operatorname{Squ}_{a}(B)$ from which is issued at least one ray crossing $C_{t}$. The notation $\mathrm{BB}$ is chosen to make apparent that the elements of $\operatorname{BB}\left(C_{t}\right)$ are considered as black boxes related to $C_{t}$ later.

Lemma 13. Let $B>0$ and $\rho>0$ be fixed. There exists a $c_{0}>0$ such that

$$
\mathrm{P}_{n f}\left(\text { there exists } C_{t} \in \mathrm{Squ}_{c_{0}}^{*}, \# \mathrm{BB}\left(C_{t}\right) \geq 12\right)=o\left(n^{-\rho}\right) \text {. }
$$

Proof. Note that, under $\mathrm{P}_{n M_{f}}$, since $\boldsymbol{S} \cap C_{1}$ and $\boldsymbol{S} \cap C_{2}$ are independent variables for $C_{1} \cap C_{2}=$ $\varnothing$, for any fixed $j \in\{1,2,3,4\}$, any $C_{t}$, and $i$ fixed, the family of variables $\left(\operatorname{Numb}_{i}\left(C, C_{t}\right)\right.$, $\left.C \in \operatorname{Squ}_{a}(B)^{j}\right)$ are i.i.d. Hence, for each $j \in\{1,2,3,4\}$, the variables $\left(\mathbf{1}_{\sum_{i=1}^{3} \operatorname{Numb}_{i}\left(C, C_{t}\right) \geq 1}\right.$, $\left.C \in \mathrm{Squ}_{a}(B)^{j}\right)$ are i.i.d. Bernoulli distributed. Let

$$
\mathrm{BB}_{j}\left(C_{t}\right)=\left\{C \in \operatorname{Squ}_{a}(B)^{j}, \operatorname{Numb}\left(C, C_{t}\right) \geq 1\right\}
$$

We have, by the union bound,

$$
\begin{aligned}
& \mathrm{P}\left(\text { there exists } C_{t} \in \mathrm{Squ}_{c_{0}}^{*}, \mathrm{\# BB}_{j}\left(C_{t}\right) \geq 3\right) \\
& \quad \leq \sum_{C_{t} \in \mathrm{Squ}_{c_{0}}^{*}}\left(\begin{array}{c}
\mathrm{Squ}_{a}(B)^{j} \\
3
\end{array}\right) \mathrm{P}_{n M_{f}}\left(\operatorname{Numb}\left(C, C_{t}\right) \geq 1\right)^{3} \\
& \quad=O\left(n^{3-6 B+2 c_{0}}\right) \sup _{C \in \mathrm{Squ}_{a}(B), C_{t} \in \mathrm{Squ}_{c_{0}}^{*}} \mathrm{P}_{n M_{f}}\left(\operatorname{Numb}\left(C, C_{t}\right) \geq 1\right)^{3} .
\end{aligned}
$$

From Lemma 12, this last term is $O\left(n^{3\left(4 B+2-c_{0}\right)}\right)$. Then, this is $o\left(n^{-\rho}\right)$ for $c_{0}$ chosen large enough. Now, to conclude, write

$$
\begin{aligned}
& \mathrm{P}_{n f}\left(\text { there exists } C_{t} \in \mathrm{Squ}_{c_{0}}^{*}, \# \mathrm{BB}\left(C_{t}\right) \geq 12\right) \\
& \quad \leq \sum_{j=1}^{4} \mathrm{P}_{n M_{f}}\left(\text { there exists } C_{t} \in \mathrm{Squ}_{c_{0}}^{*}, \# \mathrm{BB}_{j}\left(C_{t}\right) \geq 3\right) .
\end{aligned}
$$

Proof of Proposition 5. First observe that rays are defined by at most three points belonging to a ball of radius at most Navmax. From Lemma 2 we know that Navmax $\leq n^{-1 / 2+B} / 4$ with high probability. Hence, the probability that 12 rays cross a square $C_{t}$ is bounded by

$$
\left.\mathrm{P}_{n M_{f}} \text { (there exists } C_{t} \in \mathrm{Squ}_{c_{0}}^{*}, \# \mathrm{BB}\left(C_{t}\right) \geq 12\right)+\mathrm{P}_{n f}\left(\operatorname{Navmax} \geq \frac{1}{4} n^{-1 / 2+B}\right),
$$

which is $O\left(n^{-\rho}\right)$, as required, for $B>0$ and large enough $c_{0}$. This means that, for any target $t$ and any point $s$ outside these 12 squares plus the square of $\operatorname{Squ}_{a}(B)$ that contains $B\left(t, n^{-1 / 2+B}\right)$, $\boldsymbol{X}(s, t)=\boldsymbol{X}\left(s, t_{g}\right)$. For each Path $(s, t)$ and each $C$ of these (at most) 13 squares, we consider the subpath $P_{C}$ (of Path $(s, t)$ ) between the first stage that is inside $C$ and the last stage that is inside $C$. Each of these portions of the trajectory forms a black box.

For every point $s^{\prime}$ of the path $\operatorname{Path}(s, t)$ outside each of these 13 black boxes, none of the rays of the navigation star of $s^{\prime}$ crosses the square $C_{t}$; hence, $\boldsymbol{X}\left(s^{\prime}, t\right)=\boldsymbol{X}\left(s^{\prime}, t_{g}\right)$. Hence, the path is partitioned into at most 13 black boxes and at most 13 sections. 


\section{Appendix A}

Proof of Lemma 1. By hypothesis, for a certain triangular array $\left(c_{n}^{\prime}(j), j=1, \ldots,\left\lfloor\lambda / a_{n}\right\rfloor\right)$ satisfying $\left|c_{n}^{\prime}(j)\right| \leq c_{n}$ for any $j \leq\left\lfloor\lambda / a_{n}\right\rfloor$, the following representation of $y_{n}$ holds:

$$
y_{n}\left((j+1) a_{n}\right)=y_{n}\left(j a_{n}\right)+a_{n} G\left(y_{n}\left(j a_{n}\right)\right)+a_{n} c_{n}^{\prime}(j) .
$$

Hence, $y_{n}$ appears to be a perturbed version of the explicit Euler scheme, used to approximate a solution of $\operatorname{Eq}(G, z)$, which is defined by $z_{n}(0)=z$,

$$
z_{n}\left((j+1) a_{n}\right)=z_{n}\left(j a_{n}\right)+a_{n} G\left(z_{n}\left(j a_{n}\right)\right), \quad j \leq\left\lfloor\lambda / a_{n}\right\rfloor .
$$

We review here the standard argument leading to the conclusion, namely the convergence of $\left(z_{n}\right)$ to $y_{\text {sol }}$ with a bound on the speed of convergence, and derive the same result for $\left(y_{n}\right)$. First, by the Cauchy-Lipschitz lemma, $\operatorname{Eq}(G, z)$ has a unique solution, denoted by $y_{\text {sol }}$ on $[0, \lambda]$ (for $\lambda<\lambda(G, z)$, the maximum domain on which one can extend this solution). Now define, for any $x \in[0, \lambda]$

$$
R_{a_{n}}(x)=a_{n}^{-1}\left(y_{\mathrm{sol}}\left(x+a_{n}\right)-y_{\mathrm{sol}}(x)\right)-G\left(y_{\mathrm{sol}}(x)\right) .
$$

Assume that $G$ is $\alpha$-Lipschitz and bounded by $\beta$ (it is bounded since it is the maximum of a Lipschitz function on the bounded domain $D$ ); then $y_{\text {sol }}$ is clearly $\beta$-Lipschitz. We have

$$
\begin{aligned}
\sup _{x \in\left[0, \lambda-a_{n}\right]}\left|R_{a_{n}}(x)\right| & =\sup _{x \in\left[0, \lambda-a_{n}\right]}\left|\int_{x}^{x+a_{n}} \frac{G\left(y_{\mathrm{sol}}(u)\right)}{a_{n}} \mathrm{~d} u-G\left(y_{\mathrm{sol}}(x)\right)\right| \\
& \leq \sup _{x \in\left[0, \lambda-a_{n}\right]} \alpha \int_{x}^{x+a_{n}} \frac{\left|y_{\mathrm{sol}}(u)-y_{\mathrm{sol}}(x)\right|}{a_{n}} \mathrm{~d} u \\
& \leq \frac{\alpha \beta}{2} a_{n} .
\end{aligned}
$$

Let $\zeta_{n}$ be a perturbation of the explicit Euler scheme:

$$
\zeta_{n}\left((j+1) a_{n}\right)=\zeta_{n}\left(j a_{n}\right)+a_{n} G\left(\zeta_{n}\left(j a_{n}\right)\right)+\varepsilon_{n}(j), \quad j \leq\left\lfloor\lambda / a_{n}\right\rfloor .
$$

We have

$$
\begin{aligned}
\zeta_{n}\left((j+1) a_{n}\right)-z_{n}\left((j+1) a_{n}\right)= & \zeta_{n}\left(j a_{n}\right)-z_{n}\left(j a_{n}\right)+a_{n}\left(G\left(\zeta_{n}\left(j a_{n}\right)\right)-G\left(z_{n}\left(a_{n}\right)\right)\right) \\
& +\varepsilon_{n}(j), \\
\left|\zeta_{n}\left((j+1) a_{n}\right)-z_{n}\left((j+1) a_{n}\right)\right| \leq & \left(1+\alpha a_{n}\right)\left|\zeta_{n}\left(j a_{n}\right)-z_{n}\left(j a_{n}\right)\right|+\left|\varepsilon_{n}(j)\right|,
\end{aligned}
$$

and, therefore, for any $j$,

$$
\left|\zeta_{n}\left(j a_{n}\right)-z_{n}\left(j a_{n}\right)\right| \leq\left(1+\alpha a_{n}\right)^{j}\left|\zeta_{n}(0)-z_{n}(0)\right|+\sum_{m=0}^{j-1}\left(1+\alpha a_{n}\right)^{j-1-m}\left|\varepsilon_{n}(m)\right|,
$$

which yields (using $\left(1+\alpha a_{n}\right)^{k} \leq \mathrm{e}^{k a_{n}}$ for any $k \geq 0$ ), for $j$ such that $j \leq\left\lfloor\lambda / a_{n}\right\rfloor+1$,

$$
\begin{aligned}
\left|\zeta_{n}\left(j a_{n}\right)-z_{n}\left(j a_{n}\right)\right| & \leq \mathrm{e}^{j a_{n}}\left(\left|\zeta_{n}(0)-z_{n}(0)\right|+\sum_{m=0}^{j-1}\left|\varepsilon_{n}(m)\right|\right) \\
& \leq \mathrm{e}^{a_{n}\left(1+\left\lfloor\lambda / a_{n}\right\rfloor\right)}\left(\left|\zeta_{n}(0)-z_{n}(0)\right|+\sum_{m=0}^{\left\lfloor\lambda / a_{n}\right\rfloor}\left|\varepsilon_{n}(m)\right|\right) .
\end{aligned}
$$


Now to end, observe that

$$
y_{\mathrm{sol}}\left((j+1) a_{n}\right)=y_{\mathrm{sol}}\left(j a_{n}\right)+a_{n} G\left(y_{\mathrm{sol}}\left(j a_{n}\right)\right)+a_{n} R_{a_{n}}\left(j a_{n}\right) .
$$

This is a perturbed version of the Euler explicit scheme, and so, by (31), for large enough $n$,

$$
\sup _{j, j \leq\left\lfloor\lambda / a_{n}\right\rfloor+1}\left|y_{\mathrm{sol}}\left(j a_{n}\right)-z_{n}\left(j a_{n}\right)\right| \leq \mathrm{e}^{a_{n}\left(1+\left\lfloor\lambda / a_{n}\right\rfloor\right)} \sum_{m=0}^{\left\lfloor\lambda / a_{n}\right\rfloor} \frac{a_{n}^{2} \alpha \beta}{2} \leq \lambda \mathrm{e}^{\lambda} \alpha \beta a_{n},
$$

since $\mathrm{e}^{a_{n}}<2$ for large enough $n$.

The same analysis, using the comparison between $z_{n}$ and $y_{n}$ yields, for large enough $n$,

$\sup _{j,\left\lfloor\lambda / a_{n}\right\rfloor+1}\left|y_{n}\left(j a_{n}\right)-z_{n}\left(j a_{n}\right)\right| \leq \mathrm{e}^{a_{n}\left(1+\left\lfloor\lambda / a_{n}\right\rfloor\right)} \sum_{m=0}^{\left\lfloor\lambda / a_{n}\right\rfloor} a_{n} c_{n}^{\prime}(j) \leq 2 \mathrm{e}^{\lambda} \lambda \max _{j \leq \lambda / a_{n}}\left|c_{n}^{\prime}(j)\right| \leq 2 \lambda \mathrm{e}^{\lambda} c_{n}$.

Finally, summing (32) and (33), we obtain $\sup _{x \in[0, \lambda]}\left|y_{\text {sol }}(x)-y_{n}(x)\right| \leq C_{\lambda} \max \left\{a_{n}, c_{n}\right\}$ for $C_{\lambda}=\lambda \mathrm{e}^{\lambda}(2+\alpha \beta)+3 \beta$ (this is obtained at first for the points $x \in\left\{j a_{n}, j \leq\left\lfloor\lambda / a_{n}\right\rfloor+1\right\}$, then for all $x$ in the interval using the fact that on an interval of size $a_{n}$ the fluctuations of $y_{\text {sol }}$ are bounded by $a_{n} \beta$, and those of $y_{n}$ are bounded by $\beta a_{n}+c_{n}$. Then, the map $\lambda \mapsto C_{\lambda}$ has the properties stated in the lemma.

Proof of Corollary 1. Let $\left(\boldsymbol{Z}_{n}\right)$ be a sequence of processes satisfying the hypothesis. Define, for each $n$, a process $\boldsymbol{Y}_{n}$ coinciding with $\boldsymbol{Z}_{n}$ at the points $\left(j a_{n}, j=0, \ldots,\left\lfloor\lambda / a_{n}\right\rfloor+1\right)$, and which is linear between them. Introduce the event

$$
\Omega_{n}:=\bigcap_{j=0}^{\left\lfloor\lambda / a_{n}\right\rfloor}\left\{\left|\frac{\boldsymbol{Y}_{n}\left((j+1) a_{n}\right)-\boldsymbol{Y}_{n}\left(j a_{n}\right)}{a_{n}}-G\left(\boldsymbol{Y}_{n}\left(j a_{n}\right)\right)\right| \leq c_{n}\right\} .
$$

According to Lemma $1, \sup _{x \in[0, \lambda]}\left|\boldsymbol{Y}_{n}(x)-y_{\text {sol }}(x)\right| \leq D_{\lambda} \max \left\{a_{n}, c_{n}\right\}$ on $\Omega_{n}$ for a function $\lambda \mapsto D_{\lambda}$ bounded on compact sets; moreover, by the union bound,

$$
\mathrm{P}\left(\Omega_{n}\right) \geq 1-\left(\left\lfloor\frac{\lambda}{a_{n}}\right\rfloor+1\right) a_{n} d_{n} .
$$

We then also immediately obtain $\mathrm{P}\left(\sup _{x \in[0, \lambda]}\left|\boldsymbol{Y}_{n}(x)-y_{\text {sol }}(x)\right| \leq \varepsilon\right)$ converges to 0 when $n \rightarrow+\infty$ for any $\varepsilon>0$, i.e. the convergence in probability for the uniform norm of $\boldsymbol{Y}_{n}$ to $y_{\text {sol. }}$. Now define

$$
\Omega_{n}^{\prime}:=\bigcap_{j=0}^{\left\lfloor\lambda / a_{n}\right\rfloor}\left\{\sup _{x \in\left[j a_{n},(j+1) a_{n}\right]}\left|\boldsymbol{Z}_{n}(x)-\boldsymbol{Z}_{n}\left(j a_{n}\right)-\left(x-j a_{n}\right) G\left(\boldsymbol{Z}_{n}\left(j a_{n}\right)\right)\right| \leq c_{n}^{\prime}\right\} .
$$

Again, by the union bound, $\mathrm{P}\left(\Omega_{n}^{\prime}\right) \geq 1-\left(\left\lfloor\lambda / a_{n}\right\rfloor+1\right) a_{n} b_{n}$. Assume now that we are on $\Omega_{n} \cap \Omega_{n}^{\prime}$. Since $\boldsymbol{Y}_{n}$ is linear in $\left[j a_{n},(j+1) a_{n}\right]$, and since $\boldsymbol{Z}_{n}$ and $\boldsymbol{Y}_{n}$ coincide at the points $\left(j a_{n}, j \geq 0\right)$, then $\boldsymbol{Z}_{n}$ also satisfies (34). Hence, for any $t \in\left[j a_{n},(j+1) a_{n}\right]$,

$$
\boldsymbol{Y}_{n}(x)=\boldsymbol{Z}_{n}\left(j a_{n}\right)+\left(x-j a_{n}\right) G\left(\boldsymbol{Z}_{n}\left(a_{n}\right)\right) .
$$


Thus, on $\Omega_{n}^{\prime}$, we have $\left|\boldsymbol{Z}_{n}(x)-\boldsymbol{Y}_{n}(x)\right| \leq c_{n}^{\prime}$ for any $x \in[0, \lambda]$, and so $\left|\boldsymbol{Z}_{n}(x)-y_{\text {sol }}(x)\right| \leq$ $c_{n}^{\prime}+D_{\lambda} \max \left\{a_{n}, c_{n}\right\}$ on $\Omega_{n} \cap \Omega_{n}^{\prime}$. Therefore,

$$
\begin{aligned}
\mathrm{P}\left(\sup _{x \in[0, \lambda]}\left|Z_{n}(x)-y_{\mathrm{sol}}(x)\right| \leq c_{n}^{\prime}+D_{\lambda} \max \left\{a_{n}, c_{n}\right\}\right) & \geq 1-\left(\left\lfloor\frac{\lambda}{a_{n}}\right\rfloor+1\right) a_{n}\left(b_{n}+d_{n}\right) \\
& \geq 1-\left(\lambda+a_{n}\right)\left(b_{n}+d_{n}\right) .
\end{aligned}
$$

\section{References}

[1] Aldous, D. J. (2009). The shape theorem for route-lengths in connected spatial networks on random points. Preprint. Available at http://arvix.org/abs/0911.5301v1.

[2] Aldous, D. J. And Kendall, W. S. (2008). Short-length routes in low-cost networks via Poisson line patterns. Adv. Appl. Prob. 40, 1-21.

[3] Aldous, D. J. And Shun, J. (2010). Connected spatial networks over random points and a route-length statistic. Statist. Sci. 25, 275-288.

[4] Athreya, S., Roy, R. and Sarkar, A. (2008). Random directed trees and forest-drainage networks with dependence. Electron. J. Prob. 13, 2160-2189.

[5] Baccelli, F. and Bordenave, C. (2007). The radial spanning tree of a Poisson point process. Ann. Appl. Prob. 17, 305-359.

[6] Bhatt, A. G. and Roy, R. (2004). On a random directed spanning tree. Adv. Appl. Prob. 36, 19-42.

[7] Bonichon, N., Gavoille, C., Hanusse, N. And Ilcinkas, D. (2010). Connections between theta-graphs, Delaunay triangulations, and orthogonal surfaces. In Graph Theoretic Concepts in Computer Science (Lecture Notes Comput. Sci. 6410), Springer, Berlin, pp. 266-278.

[8] Bordenave, C. (2008). Navigation on a Poisson point process. Ann. Appl. Prob. 18, 708-746.

[9] Bose, P. et al. (2010). Pi/2-angle Yao graphs are spanners. ArXiv e-prints, 2010, 1001.2913. Preprint. Available at http://arvix.org/abs/1001.2913v1.

[10] Clarkson, K. L. (1987). Approximation algorithms for shortest path motion planning. In STOC '87: Proc. 19th Annual ACM Symp. on Theory of Computing, ACM, New York, pp. 56-65.

[11] Ferrari, P. A., Fontes, L. R. G. And Wu, X.-Y. (2005). Two-dimensional Poisson trees converge to the Brownian web. Ann. Inst. H. Poincaré Prob. Statist. 41, 851-858.

[12] Kallenberg, O. (1997). Foundations of Modern Probability. Springer, New York.

[13] KeIL, J. M. (1988). Approximating the complete Euclidean graph. In SWAT 88 (Halmstad, 1988; Lecture Notes Comput. Sci. 318), Springer, Berlin, pp. 208-213.

[14] LI, X.-Y., WAN, P.-J. AND WANG, Y. (2001). Power efficient and sparse spanner for wireless ad hoc networks. In Proc. IEEE 10th Internat. Conf. on Computer Communications and Networks, pp. 564-567.

[15] O'Rourke, J. (2010). The Yao graph $Y_{6}$ is a spanner. Preprint. Available at http://arxiv.org/abs/1003.3713v2.

[16] Penrose, M. D. And Wade, A. R. (2006). On the total length of the random minimal directed spanning tree. Adv. Appl. Prob. 38, 336-372.

[17] Petrov, V. V. (1975). Sums of Independent Random Variables. Springer, New York.

[18] Ruppert, J. and Seidel, R. (1991). Approximating the $d$-dimensional complete Euclidean graph. In $3 r d$ Canadian Conf. Computational Geometry, pp. 207-210.

[19] YAO, A. C. (1982). On constructing minimum spanning trees in $k$-dimensional spaces and related problems. SIAM J. Comput. 11, 721-736. 